EXPERIMENTAL INVESTIGATION OF NOZZLE SPACING EFFECTS ON CHARACTERISTICS OF ROUND TWIN FREE JETS

by

Andrew Laban

A Thesis submitted to the Faculty of Graduate Studies of The University of Manitoba in partial fulfilment of the requirements of the degree of

\title{
MASTER OF SCIENCE
}

Department of Mechanical Engineering

University of Manitoba

Winnipeg

Copyright (C) 2018 by Andrew Laban 


\begin{abstract}
The objective of this research is to investigate the effects of nozzle spacing on the mean velocity and higher order turbulent statistics of free round twin jets produced from sharp contraction nozzles. The experiments were performed in an air chamber for four nozzle spacing ratios, $S / d=2.8,4.1,5.5$ and 7.1 and at a fixed Reynolds number of 10,000. A planar particle image velocimetry system was used to conduct the velocity measurements. The results show that downstream of the potential core, a reduction in spacing ratio leads to an earlier and more intense interaction between the jets, indicated by enhanced half-velocity width spread rate in the inner shear layers and a significant rise of turbulent intensities and vorticity thickness along the symmetry plane. A reduction in spacing ratio, however, diminishes the ambient fluid entrainment along the inner shear layers leading to reduced core jet velocity decay rate. A closer proximity of the jets also leads to the suppression of Reynolds stresses in the inner shear layers but not in the outer shear layers, suggesting that there exists no significant communication between the inner and outer shear layers. The Reynolds stress ratios along the jet centerline reveals the highest anisotropy in the potential core region. Skewness and flatness factors are used to examine the asymmetry and intermittency of the velocity fluctuations, while two-point correlation analysis is employed to investigate the effects of nozzle spacing ratio on the spatial coherence of large-scale vortical structures.
\end{abstract}




\section{ACKNOWLEDGMENT}

I would like to express my sincere gratitude to my academic advisor Dr. Mark F. Tachie for his invaluable support and guidance during this research.

I would also like to acknowledge the financial support of the Natural Sciences and Engineering Research Council of Canada and the University of Manitoba. 


\section{DEDICATION}

This thesis is dedicated to my parents, Laban and Seham Eskaors, for training me to be humble and hardworking, and to my true love, Katrina Laban, for supporting me day and night to see this program to completion. 


\section{TABLE OF CONTENTS}

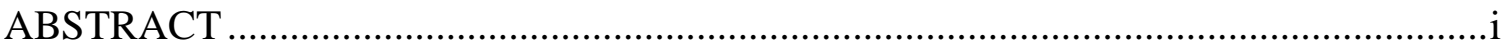

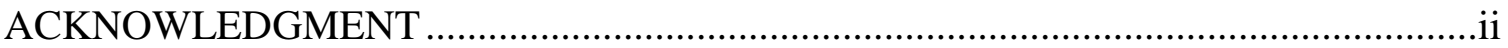

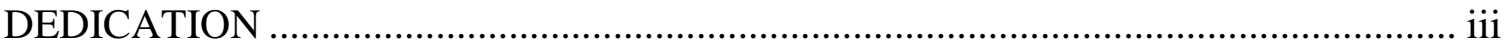

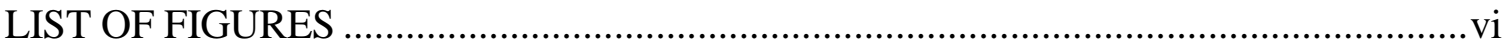

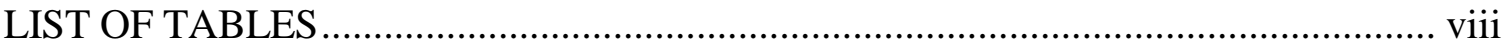

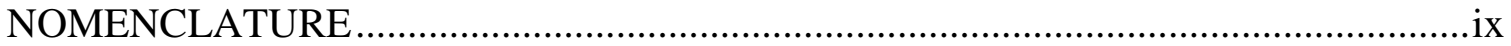

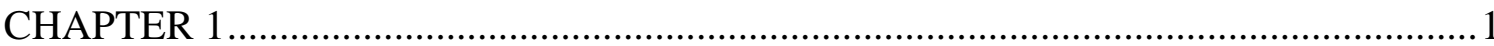

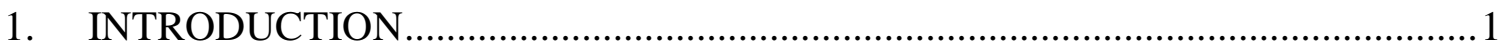

1.1. Motivation and Objective of Research .........................................................

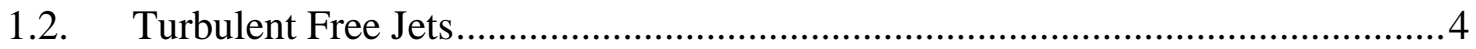

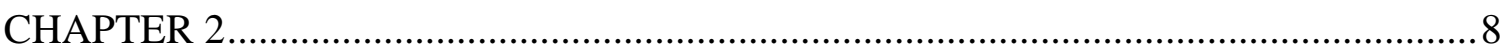

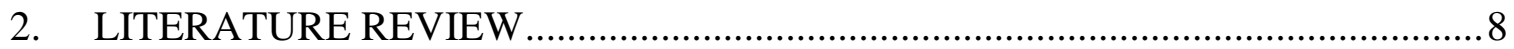

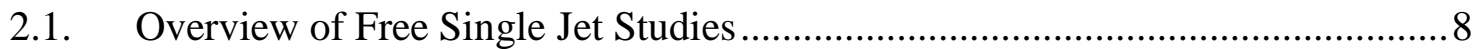

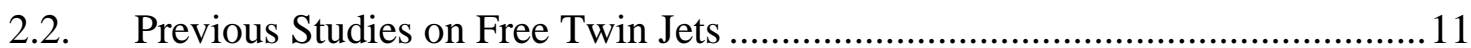

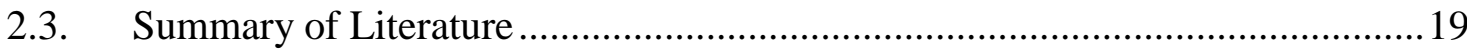

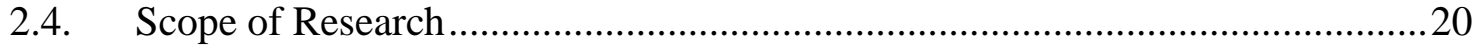

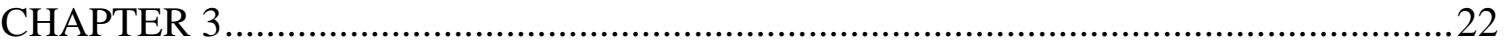

3. EXPERIMENTAL SETUP AND MEASURMENT PROCEEDURE .....................22

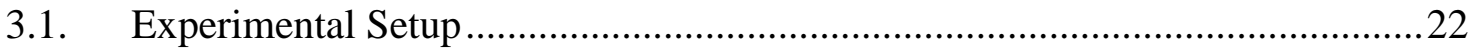

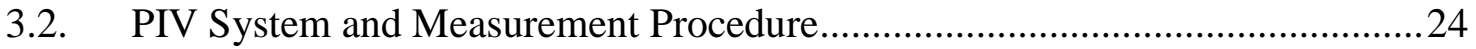

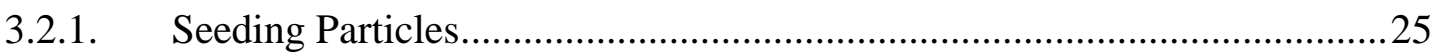

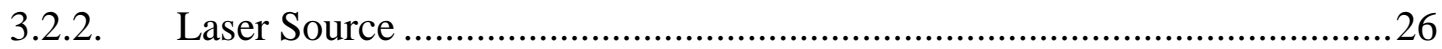


3.2.3. Recording Medium

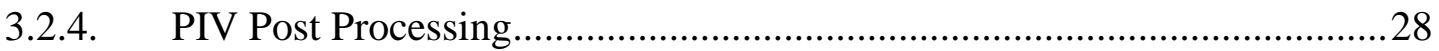

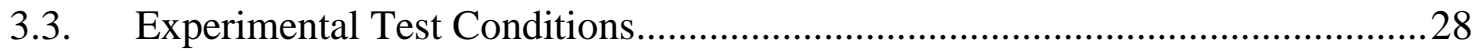

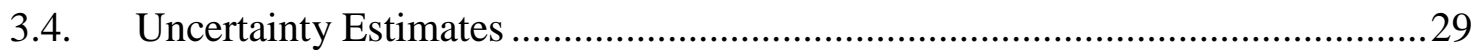

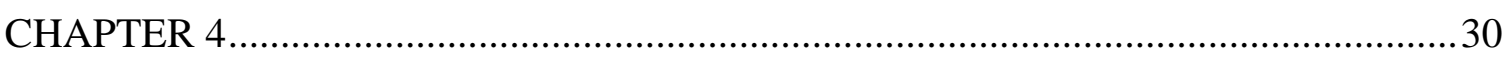

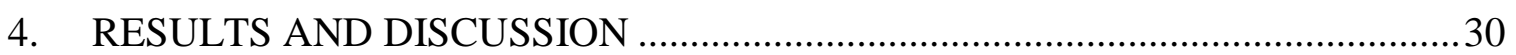

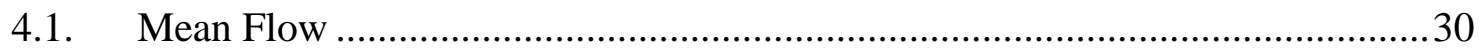

4.2. Reynolds Stresses, Turbulent Intensities and Turbulent Kinetic Energy ..........41

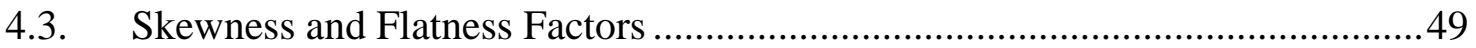

4.4. Mean Velocities and Reynolds Stresses across the Shear Layers.......................52

4.5. Two-point Correlation and Integral Length Scales.........................................54

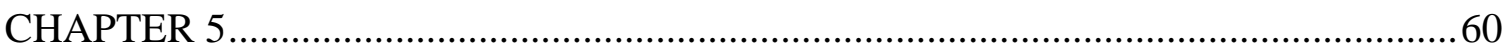

5. CONCLUSIONS AND RECOMMENDATION FOR FUTURE WORK ...............60

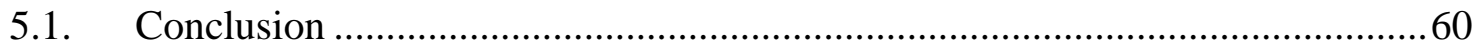

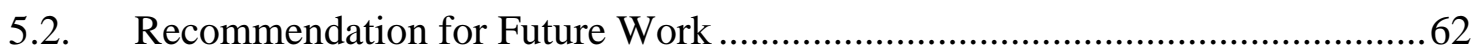

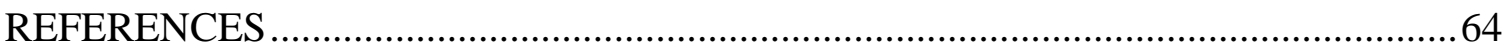

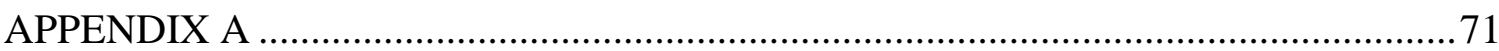

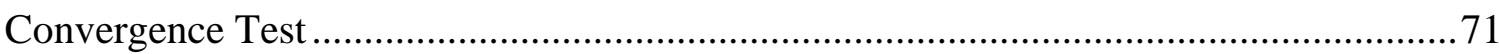

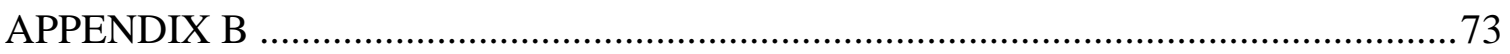

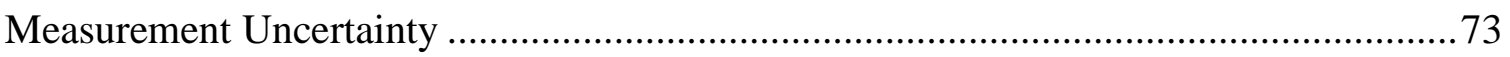

B.1 Systematic and Random Error Analysis Procedure .......................................... 73

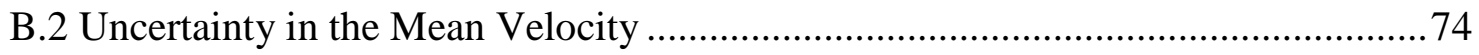




\section{LIST OF FIGURES}

Figure 1.1: Air flow diagram of a typical active beam in cooling [1] 2

Figure 1.2 Schematic of free single jet configuration 5

Figure 1.3: Schematic of twin jet configuration $\quad 7$

Figure 3.1: (a) Schematic and (b) picture of air chamber test facility 23

Figure 3.2: Test round nozzle with a modified contraction 24

Figure 4.1: Contours of streamwise and transverse mean velocity for $S / d=2.8(\mathrm{a}, \mathrm{b}), 4.2$

(c, d ) and $5.5(\mathrm{e}, \mathrm{f})$ and $7.1(\mathrm{~g}, \mathrm{~h})$

Figure 4.2: (a) Symmetry plane and jet centerline streamwise velocity downstream evolution, (b) Symmetry plane streamwise velocity normalized by $U_{c l}$ and $x_{m p}$

Figure 4.3: (a) Merging point, (b) combined point locations for present and previous twin jet studies

Figure. 4.4: Streamwise evolution of jet centerline velocity normalized by the maximum exit velocity

Figure 4.5: (a) Outer shear layer half-velocity spread (b) inner shear layer half-velocity $\begin{array}{ll}\text { spread } & 38\end{array}$

Figure 4.6: Local vorticity thickness along the jet's inner shear layer

Figure 4.7: Contours of Reynolds shear stress, streamwsie Reynolds normal stress and transverse Reynolds normal stress for $S / d=(\mathrm{a}, \mathrm{b}, \mathrm{c}) 2.8$, (d, e, f) 4.1, (g, h, i) 5.5 and $(\mathrm{j}, \mathrm{k}, \mathrm{l}) 7.1$

Figure 4.8: Contours of turbulent kinetic energy, production, convection and turbulent diffusion for $S / d=2.8(\mathrm{a}, \mathrm{c}, \mathrm{e}, \mathrm{g})$ and $7.1(\mathrm{~b}, \mathrm{~d}, \mathrm{f}, \mathrm{h})$ normalized by $U_{\max } / d 44$ 
Figure 4.9: Development of (a) streamwise turbulent intensity, $u_{r m s} / U_{\max }$ and (b) transverse turbulent intensity, $v_{\text {rms }} / U_{\max }$ along symmetry plane and jet centerline

Figure 4.10: (a) Development of $u_{r m s}$ and (b) $v_{r m s}$ normalized by $U_{c l}$ along the jet centerline

Figure 4.11: Scaling of (a) $u_{r m s}$ and (b) $v_{r m s}$ along the symmetry plane

Figure 4.12: Reynolds normal stresses ratio along jet centerline

Figure 4.13: Streamwise and transverse mean velocity (a) skewness ( $S u$ and $S v)$ and (b) flatness $(F u$ and $F v)$ along the jet centerline

Figure 4.14: Streamwise and transverse mean velocity (a) skewness ( $S u$ and $S v)$ and (b) flatness $(F u$ and $F v)$ along the symmetry plane

Figure 4.15: One-dimensional profiles of dimensionless (a) streamwise velocity (b) Reynolds shear stresses (c) streamwise normal stresses and (d) transverse normal stresses across select streamwise locations

Figure 4.16: Iso-contours of the two-point correlation of streamwise velocity fluctuation, $R_{u u}$ and transverse velocity fluctuation, $R_{v v}$ at the merging point for $S / d=$ $2.8(\mathrm{a}, \mathrm{b}), 4.1(\mathrm{c}, \mathrm{d}), 5.5(\mathrm{e}, \mathrm{f})$ and $7.1(\mathrm{~g}, \mathrm{~h})$

Figure 4.17: One-dimensional profiles of (a) $R_{u u}(x)$ and (b) $R_{v v}(y)$ at the merging point locations.

Figure 4.18: Variation of the (a) streamwise and (b) transverse integral length scales along the jet centerline

Figure 4.19: Variation of the (a) streamwise and (b) transverse integral length scales along the outer (solid symbols) and inner (open symbols) half-velocity widths 
Figure A.1 Profiles of dimensionless (a) streamwise mean velocity, (b) Reynolds shear stress, (c,d) Reynolds normal stresses, (e)skewness, and (f) flatness factors for $N=1,000,2,000,3,000,4,000,5,000$ and 7,500 at $x / d=3$ and 7

\section{LIST OF TABLES}

Table 2.1 Summary of test conditions, measurement technique and flow parameters analyzed in relevant free twin jet studies

Table 4.1: Streamwise mean velocity peak values and locations along the symmetry

plane

Table 4.2: Summary of fitting coefficients for streamwise mean velocity scaling along the symmetry plane according to Eqn. 4.1

Table 4.3 Summary of jet centerline decay rates before and after the combined point $\quad 38$

Table 4.4: Summary of half-velocity width spread rates along the jet's outer and inner shear layers

Table 4.5 Vorticity thickness growth linear growth rate along the jet's inner shear layer 40

Table 4.6 Summary of fitting coefficients for streamwise and transverse turbulent intensities' scaling along the symmetry plane according to Eqn. (4.1)

Table 4.7 Streamwise and transverse integral length scales at merging point locations

Table B.1 Systematic uncertainty limits of mean velocity at the jets' symmetry plane $(x / d$

$$
=8 \& y / d=0)
$$

Table B.2 Random uncertainty of mean velocity at the jets' symmetry plane $(x / d=8 \&$

$$
y / d=0)
$$




\section{NOMENCLATURE}

\section{English}

$C k^{*} \quad$ Turbulent convection normalized by $U_{c l}$

$d \quad$ Nozzle diameter

$d_{p} \quad$ Seeding particle object diameter

$d_{\text {image }} \quad$ Seeding particle image diameter

$d_{\text {pitch }} \quad$ Pixel pitch

$D k^{*} \quad$ Turbulent diffusion normalized by $U_{c l}$

$f_{\#} \quad$ Camera focal number

$F u_{c l} \quad$ Flatness of streamwise velocity along jet centerline

$F u_{\text {sym }} \quad$ Flatness of streamwise velocity along symmetry plane

$k^{*} \quad$ Turbulent kinetic energy normalized by $U_{c l}$

$K_{s+0.5} \quad$ Jet's outer shear layer spread rate according to $y_{+0.5}$

$K_{s-0.5} \quad$ Jet's inner shear layer spread rate according to $y_{-0.5}$

$L_{0} \quad$ Digital image width in pixels

$L_{i} \quad$ Field of view width of the camera in meters

$K_{u} \quad$ Jet centerline velocity decay rate

M Magnification factor

$P k^{*} \quad$ Turbulent production normalized by $U_{c l}$

$P_{c} \quad$ Potential core length

Re Reynolds number

$S \quad$ Nozzle spacing

$S u_{c l} \quad$ Skewness of streamwise velocity along jet centerline

$S u_{s y m} \quad$ Skewness of streamwise velocity along symmetry plane 


\begin{tabular}{|c|c|}
\hline$S v_{c l}$ & Skewness of transverse velocity along jet centerline \\
\hline$S v_{\text {sym }}$ & Skewness of transverse velocity along symmetry plane \\
\hline$t_{s}$ & Seeding particle's response time \\
\hline$U_{s}$ & Seeding particle settling velocity \\
\hline$U_{\max }$ & Maximum jet exit velocity \\
\hline$U^{*} \max$ & Maximum jet exit velocity normalized by $U_{c l}$ \\
\hline$U_{\text {sym }}$ & Symmetry plane streamwise velocity \\
\hline$U^{*}{ }_{\text {sym }}$ & Symmetry plane streamwise velocity normalized by $U_{c l}$ \\
\hline$\left(U^{*}{ }_{s y m}\right)_{\max }$ & Maximum value of symmetry plane streamwise velocity normalized by $U_{c l}$ \\
\hline$U_{c l}$ & Jet centerline velocity \\
\hline$U^{*}$ & Streamwise mean velocity normalized by $U_{c l}$ \\
\hline$\mu_{f}$ & Fluid dynamic viscosity \\
\hline$u_{r m s, c l}^{*}$ & Streamwise turbulent intensity along jet centerline normalized by $U_{c l}$ \\
\hline$\overline{u^{2 *}}$ & Streamwise Reynolds normal stress normalized by $U_{c l}$ \\
\hline$\overline{u_{c l}^{2}}$ & Streamwise Reynolds normal along jet centerline \\
\hline$\overline{u v *}$ & Reynolds shear stress normalized by $U_{c l}$ \\
\hline$l_{r m s, s y m}^{*}$ & Streamwise turbulent intensity along symmetry plane normalized by $U_{c l}$ \\
\hline$V^{*}$ & Transverse mean velocity normalized by $U_{c l}$ \\
\hline$v_{r m s, c l}^{*}$ & Transverse turbulent intensity along jet centerline normalized by $U_{c l}$ \\
\hline$\rho_{r m s, s y m}^{*}$ & Transverse turbulent intensity along symmetry plane normalized by $U_{c l}$ \\
\hline$\overline{v^{2 *}}$ & Transverse Reynolds normal stress normalized by $U_{c l}$ \\
\hline$\overline{v_{c l}^{2}}$ & Transverse Reynolds normal along jet centerline \\
\hline$d \delta_{w} / d x$ & Instantons rate of change of vorticity thickness \\
\hline
\end{tabular}




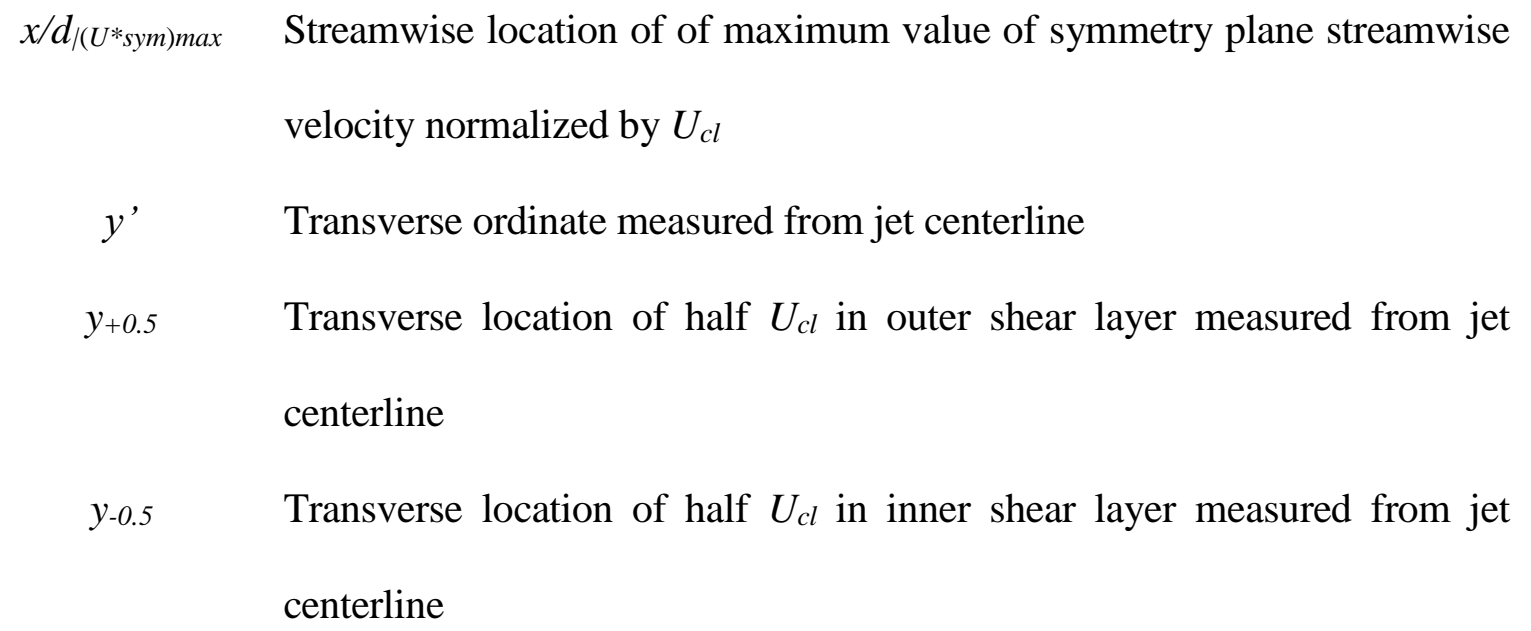

\section{Greek}

$\begin{array}{cl}\rho_{p} & \text { Seeding particle density } \\ \rho_{f} & \text { Fluid density } \\ \Delta s & \text { Particle displacement between two consecutive digital images } \\ \Delta t & \text { Time separation between laser pulses } \\ \mu_{f} & \text { Fluid dynamic viscosity } \\ v_{f} & \text { Fluid kinematic vicousity } \\ \lambda & \text { Laser wavelength } \\ \delta_{w} & \text { vorticity thickness } \\ d \delta_{w} / d x & \text { Instantons rate of change of vorticity thickness }\end{array}$

\section{Acronyms}

CFD Computational fluid dynamics

FFT Fast Fourier transform

HWA Hot wire anemometry

HVAC Heating, ventilation and air conditioning

LDA Laser Doppler anemometry

Nd:YAG Neodymium-doped yttrium aluminum garnet 
OP Orifice plate nozzle

PIV Particle image velocimetry

Re Reynolds number

SJ Single jet

SC Smooth contraction 


\section{CHAPTER 1}

\section{INTRODUCTION}

The experimental study of three dimensional (3D) free confluent jets is of critical importance to the advancement of various engineering technologies and validation of emerging numerical models of turbulent fluid dynamics. The following sections discuss the motivation and objective of this study as well as an overview of single and twin turbulent free jets.

\subsection{Motivation and Objective of Research}

The study of free turbulent jets is fundamental to the development of many engineering systems that rely on the interaction of multiple confluent jets for their functionality. Such applications include heat transfer augmentation, film cooling, thrust producing devices, and heating, ventilation and air conditioning (HVAC) systems. In HVAC applications, for example, turbulent jets form the basis of analyzing and designing efficient induction and mixing jet ventilation systems. Large entrainment from the ambient fluid into the core jet is required for optimal performance of these ventilation systems, and scientific research is needed to identify optimal nozzle configurations that maximize induction and mixing between the core jets and the ambient fluid.

One of such ventilation systems is the active chilled beam, designed by Price Industries Ltd, a leading manufacturer of air distribution and HVAC systems in North America. This beam uses a hybrid (water/air) system to meet the balance of the sensible cooling and heating loads of a space while promoting a high level of occupant comfort and energy efficiency [1]. 


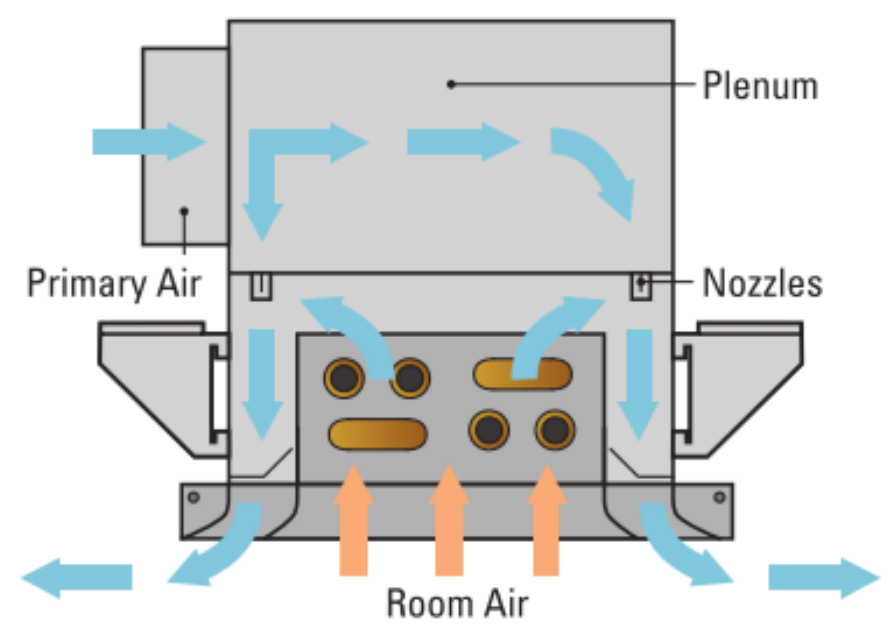

Figure 1.1: Air flow diagram of a typical active beam in cooling [1]

Figure 1.1 shows an air flow diagram of a typical active chill beam. As can be seen from the diagram, an active beam receives dry air from the primary system through a pressurized plenum. This primary supply air is then forced through induction nozzles to create a high velocity air pattern in the area adjacent to the coil. The high velocity air pattern causes a reduction in the local static pressure, which in turn induces room air through the heating/cooling coil. The induced air then mixes with the primary supply air and is discharged back into the space via slots along the beam. The induction nozzles that supply the primary air play an important role in the induction of the room air, and therefore an innovative design of the geometry and spacing of these nozzles is critical. The interaction between the individual air jets issued from these nozzles can lead to significantly more complex turbulent transport phenomena than are observed in prototypical single turbulent free jets. While the mixing and turbulence characteristics of free single jets have been experimentally investigated in considerable detail, far less is documented on multiple turbulent free jets.

In the design of the active chilled beam, the Research and Development group at Price Industries Ltd. employed prototype testing and Computational Fluid Dynamics (CFD) technique. While the CFD simulations provide performance data and qualitative information 
inside the chilled beam, there are no reliable experimental data of the flow inside of the beam to validate the numerical results. As a consequence, the accuracy of the numerical models used in the simulations could not be verified. In view of these fundamental challenges, Price and the University of Manitoba embarked on a collaborative research to experimentally study the effects of nozzle geometry, spacing between adjacent nozzles and arrangement of arrays of nozzles on the mixing and induction capacity of free turbulent air jets, starting with a single jet then on to multiple jets with various spacing configurations. The experimental studies will provide a deeper understanding of the mixing characteristics and turbulent transport phenomena in multiple jets, and also provide benchmark databases to aid CFD specialists at Price Industries Ltd. in developing accurate numerical models for various ventilation systems including the active chilled beam.

The simplest configuration of multiple jets are twin jets issued from two identical parallel nozzles. Thus, as part of the collaborative research program, this research aims to investigate the mixing and turbulent characteristics of twin round jets. Specifically, the experimental study reported herein focuses on detail investigation of the effect of the nozzles' spacing on the interaction of twin round jets with each other and with the ambient environment. 


\subsection{Turbulent Free Jets}

A single free jet is the most fundamental jet flows, and knowledge of its mixing and turbulent characteristics is a prerequisite to a comprehensive understanding of the more complicated free twin jets, which are the primary focus of this research. Figure 1.2 shows a schematic of a free single round jet and defines some of the flow parameters that are used to study the flow field. The jet, with an exit velocity $U_{j}$, is issued from a round nozzle of diameter $d$. The local maximum of the streamwise mean velocity (hereafter referred to as the jet centerline, $U_{c l}$ ) of the jet decreases monotonically with streamwise distance due to entrainment of the ambient fluid into the core jet. The entrainment of the ambient fluid commences in the shear layers due to the large velocity gradient in these regions which causes vortical structures to form, evolve and pair up [2]. The regular vortex formation and pairing processes are the main mechanism of ambient fluid entrainment and consequently, decay of the jet velocity with streamwise distance from the nozzle exit. The decay of the jet is accompanied by a transverse spread into the ambient fluid. A measure of the jet's spread is the half-velocity width, which is defined as the transverse distance from the jet centerline to the location where the local streamwise mean velocity is $0.5 U_{c l}$ in the shear layer $\left(y_{0.5}\right)$. 


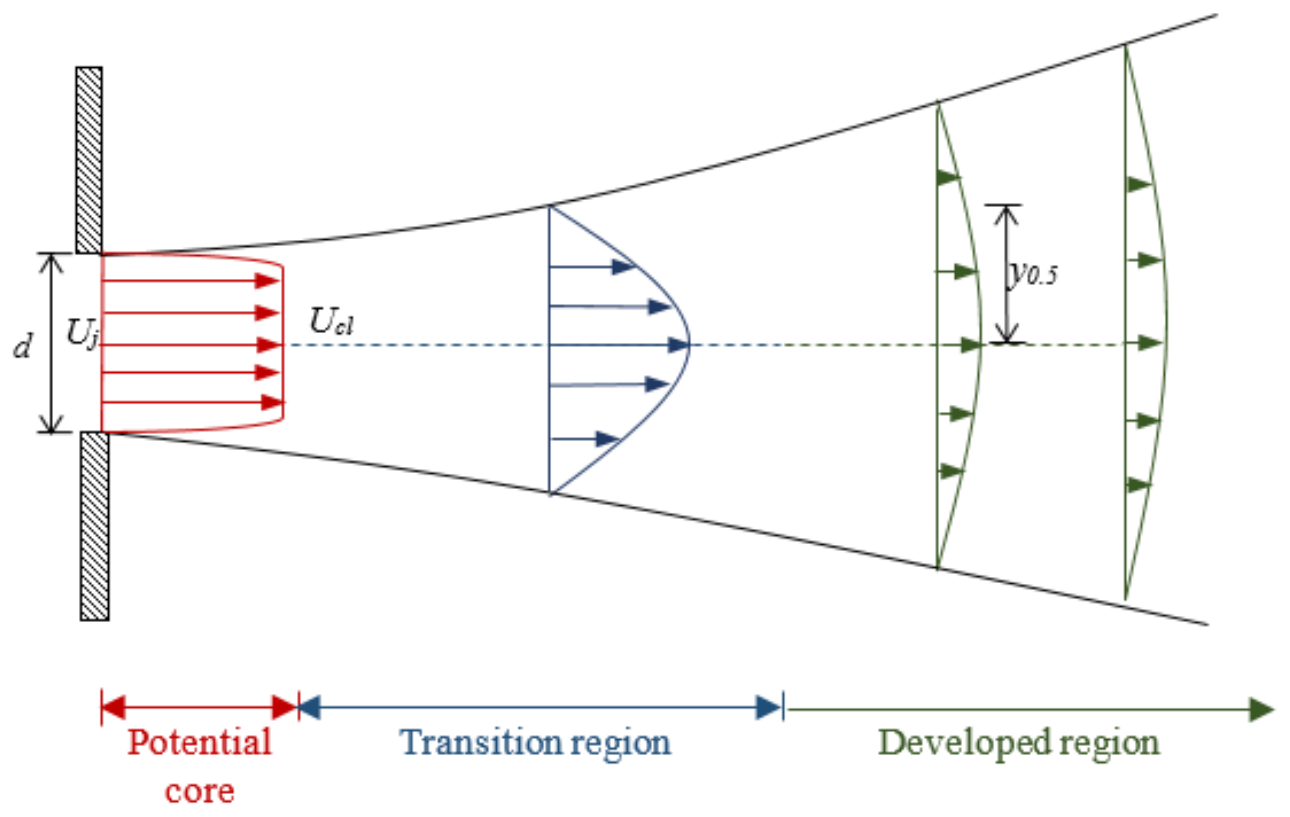

Figure 1.2 Schematic of free single jet configuration

As shown in Fig. 1.2, the evolution of a single free jet is defined by three streamwise regions: the potential core, transition (or developing) and developed (or self-similar) regions. The potential core and the early stage of the transition region are often referred to as the nearfield of the jet, whereas the later stage of the transition region and the self-similar region are referred to as the far-field of the jet. The potential core is defined as the region near the nozzle exit where the jet centerline velocity remains constant. This region usually extends to the first few nozzle diameters, and its extent is commonly used as a measure of the mixing performance of the free jet with the ambient fluid in the near field. The transition region is the region between the potential core and the developed region, which is defined as the region where the distribution of dimensionless streamwise mean velocity are self-similar. The general view point is that high order statistics such as the Reynolds stresses and triple velocity products also become self-similar in this region but at further downstream distances than the mean velocity. In the developed region, the decay of centerline mean velocity the $U_{c l}$ with streamwise distance exhibits a linear behavior, and is expressed using the following equation [3]: 


$$
\frac{U_{j}}{U_{c l}}=K_{u}\left(\frac{x}{d}-C_{0}\right)
$$

where $K_{u}$ is the decay rate and $C_{o}$ is the kinematic virtual origin of the jet.

The growth or spread of $y_{0.5}$ also exhibits a linear behavior in the developed region and is expressed using the following equation [3]:

$$
\frac{y_{0.5}}{d}=K_{s 0.5}\left(\frac{x}{d}+C_{s 0.5}\right)
$$

where $K_{s 0.5}$ is the spread rate of $y_{0.5}$ and $C_{s 0.5}$ is the geometric virtual origin of the jet.

The twin jets, on the other hand, is a combination of two closely spaced single jets that bear some similarities with the single jet but also introduce some complexities due to the mutual interaction of the jets. A schematic of twin jets and the nomenclatures that are used in this study are shown in Fig. 1.3. The jets are issued from two identical parallel nozzles with centerto-center spacing $S$. Similar to the single jet, the centerline velocities $\left(U_{c l}\right)$ of the individual jets decrease monotonically with streamwise distance and their decay is accompanied by a transverse spread, outward into the ambient fluid and inward towards the symmetry plane. The half-velocity widthis defined distinctly for the jets' outer shear layers as $y_{+0.5}$ and for the inner shear layers as $y_{-0.5}$. While the outer shear layers grow without bound, as in single free jets, the inner shear layers of twin jets spread towards each other and eventually converge on the symmetry plane at the merging point, $x_{m p}$. The region extending from the nozzle exit to the merging point is referred to as the converging region. The centerlines of the twin jets also deflect towards each other and eventually merge on the symmetry plane at the combined point, $x_{c p}$. The region between $x_{m p}$ and $x_{c p}$ is referred to as the merging region. The combined point also marks the onset of the combined region, where the twin jets are fully merged into a single jet. The streamwise mean velocity along the symmetry plane, denoted by $U_{s y m}$, and $U_{c l}$ coincide at $x_{c p}$. 


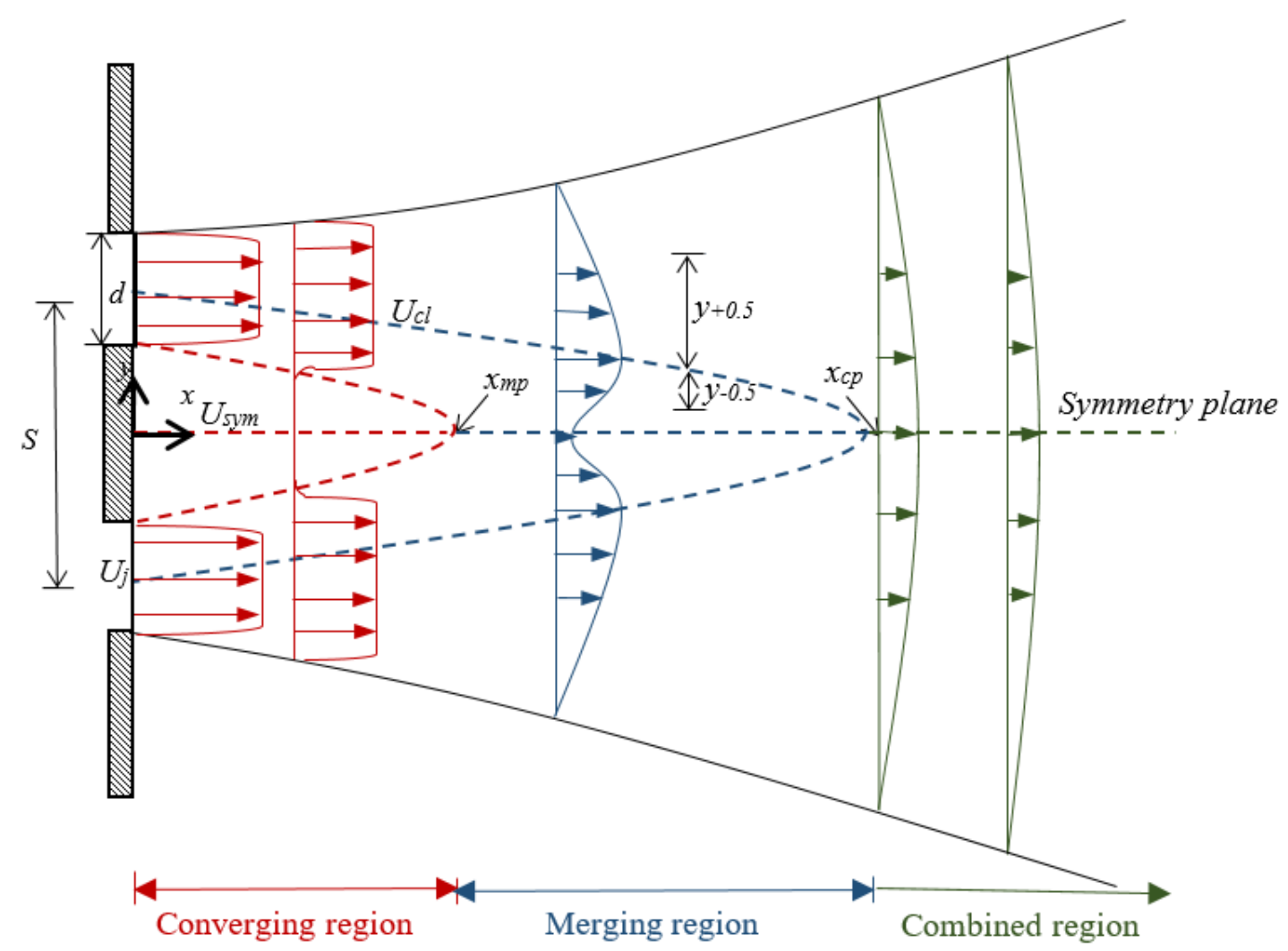

Figure 1.3: Schematic of twin jet configuration 


\section{CHAPTER 2}

\section{LITERATURE REVIEW}

This literature survey discusses previous studies on free single and twin jets, and their dependency on initial conditions such as Reynolds number $(R e)$, nozzle geometry and nozzle spacing. A summary of the literature review is followed by the scope of the present study and how it fills the knowledge gap.

\subsection{Overview of Free Single Jet Studies}

Previous studies have shown that the mean velocity and turbulent statistics of free single jets depend on $R e$ at the nozzle exit. A study by Deo et al. [4] investigated the influence of $R e$ on a plane single jet issued from a radially contoured slot nozzle at $1,500 \leq R e \leq 16,500$. Prior to the jet's fully developed region, the potential core decreased and the jet spread increased with increasing $R e$, which suggested that increasing $R e$ enhances entrainment of the ambient fluid in the near field. In the fully developed region, Eqns. (1.1) and (1.2) were used to quantify the spread and decay rates of the mean velocity. The results showed that the decay and spread rates varied from 0.22 to 0.16 , and 0.14 to 0.09 , respectively as $R e$ increased from 1,500 to 16,500. In addition, the asymptotic values of the streamwise turbulent intensity increased from 0.16 to 0.23 as $R e$ increased from 1,500 to 16,500 . In another study by Doglia et al. [5], the effects of $R e$ on the mean flow field of a free round jet issued from a smooth contraction (SC) nozzle at $R e=15,000$ and 25,000 were investigated. The potential core length was $40 \%$ longer at $R e=15,000$ than at $R e=25,000$, while the jet spread rate in the fully developed region was $58 \%$ higher at $R e=15,000$ than that at $R e=25,000$, which agrees with the trend observed for plane jets [4]. More recently, Aleyasin et al. [6] studied the mean and turbulent characteristics of single round jets issued from a sharp contraction nozzle at $R e=6,000-20,000$ in the near and intermediate regions using the same facility and measurement technique employed in the 
present study. The results showed that the potential core length actually increased by approximately $13 \%$ when $R e$ increased from 6,000 to 20,000 , in contradiction to [4] and [5]. The decay and spread rates in the fully developed region, however, decreased by $6 \%$ and $13 \%$, respectively as $R e$ increased from 6,000 to 20,000. Lastly, the streamwise and transverse turbulent intensities along the jet centerline reached an asymptotic value of 0.24 and 0.18 , respectively, irrespective of $R e$.

Most documented publications demonstrate that if $R e$ at the jet exit is greater than a few thousand, the mean and/or turbulent characteristics of single jets are independent of $R e$. For example, $\mathrm{Xu}$ et al. [7] examined the flow field of a free single jet issued from a pipe square nozzle at $8,000 \leq R e \leq 50,000$. They observed that the decay and spread rates as well as the asymptotic value of the the streamwise turbulent intensity became $R e$-independant at $R e \geq$ 30,000. Fellouah et al. [8] experimentally examined the effect of $R e$ on the mean velocity and turbulent intensities of a round single jet issued from a SC nozzle at $R e$ of 6,000, 10,000 and 30,000. It was found that the centerline velocity decay as well as the downstream profiles of the streamwise and transverse turbulent intensities across the flow field were independent of Re. A recent study by Aleyasin et al. [9], that was also performed at the same facility as the present study, investigated the mean and turbulent characterisitcs of round single jets issued from a sharp contraction nozzle. The results revealed that the spread and decay rates reached an asympototic value at $R e \geq 10,000$. These results clearly demonstrate that there is no consensus on the exact $R e$ value at which the flow characteristics become independent of $R e$ because the nozzle geometry and experimental facility play a significant role as well.

The nozzle type also plays a significant role on the behavior of free jet flow. Quinn et al. [10] investigated the mean and turbulent characteristics of a free single round jet issued from orifice plate (OP) and SC nozzles at $R e=184,000$. In the near field, the potential core length of the OP jet was $18 \%$ shorter than that of the SC jet. In the fully developed region, the jet 
decay and spread rates were approximately $2 \%$ higher for the OP jet. Moreover, the streamwise turbulence intensity along the jet centerline for the OP jet reached a 38\% higher peak and at an earlier streamwise location than its SC counterpart. The difference in the mean flow of OP and SC jets was explained by $\mathrm{Mi}$ et al. [11] who observed the OP jet had a more complex 3D underlying structure than the SC jet, which indicated the occurrence of self-induction, vortex stretching and reconnection, and hence a higher mixing with the ambient environment. Although OP nozzles generally provide superior mixing than SC nozzles, the former can generate an undesirable fluid phenomenon known as 'cavitation' [12]. When this phenomenon takes place, a flow-induced vibration can be generated leading to high level of noise and causing acoustic nuisance to the people in the vicinity [13]. In addition, Xu et al. [14] compared the mean and turbulent characteristics of single round jet issued from SC and fully developed pipe nozzles at $R e=86,000$. It was found that the decay and spread rates in the fully developed regions were respectively $14 \%$ and $10 \%$ higher for the SC nozzle. Moreover, one-dimensional profiles of Reynolds normal and shear stresses across the flow field were found to be wider for the SC nozzle, which in conjunction with the spread rate results, imply that the SC jet develops more rapidly than the pipe jet. The off-axis peaks were also shown to be larger for the SC jet.

The geometry of the exit nozzle plays a significant role in the jet development as well. Mi et al. [15] studied the centerline evolutions of mean velocity and turbulent statistics of eight OP jets issuing from different nozzle shapes, namely, round, cross, star, square, rectangle, ellipse, equilateral triangle and isosceles triangle nozzles at $R e=15,000$. The results showed that the non-circular jets exhibited approximately 13\% - 75\% shorter potential core length than observed for the round nozzle, but there was no significant variation in the far-field decay rates. In addition, the streamwise turbulent intensity along the centerline exhibited localized humps upstream of their asymptotic behavior for the triangular, rectangular and elliptic jets, the most significant of which was associated with the isosceles triangle. Humps, however, did not show 
in circular, square, cross or star-shaped jets. The authors concluded that a localized hump is associated with the axis switching phenomenon in which the jet's axes rotate while the cross section remains similar to those of the jet nozzle. More recently, an experimental study was conducted by Aleyasin et al. [16] to investigate the mean and turbulent characteristics of free single jets issued from round, square, rectangular, elliptic and triangular sharp contraction nozzles at $R e=10,000$ in the same facility as [6] and the present study. The results showed that noncircular jets had shorter potential core length and faster growth of turbulence intensity on jet centerlines compared to the round jet. Also, the spread and decay rates and the level of Reynolds stresses were the highest in the jets issuing from the elliptic and rectangular nozzles, implying their enhanced mixing.

\subsection{Previous Studies on Free Twin Jets}

Over the past decades, experiments have been conducted to understand how changes in $R e$ and nozzle spacing affect the mean velocity and turbulent characteristics of twin jets issued mostly from SC nozzles. These investigations include two-dimensional (2D) or plane twin jets [17-21] and 3D twin jets [22-25]. The vast majority of these investigations were performed using hot wire anemometry (HWA) or laser Doppler anemometry (LDA), and focused primarily on the decay and spread rates, and the mean velocities and Reynolds stresses. The most relevant of these studies are summarized in Table 2.1, where the test conditions, measurement technique and some of the key flow parameters that were analyzed in the individual studies are summarized. In this table, $K_{u}$ represents the rate at which $U_{c l}$ decays according to the Eqn. 1.1 in Section 1.2, while $K_{s+0.5}$ and $K_{s-0.5}$ represent the spread rates of $y_{+0.5}$ and $y_{-0.5}$, respectively, measured relative to the jet centerline according to Eqn. 1.2 in

Section 1.2. Moreover, $P_{c}$ represents the jet potential core length, $\overline{u^{2}}$ and $\overline{v^{2}}$ are the streamwise and transverse Reynolds normal stresses, respectively, $u_{r m s}$ and $v_{r m s}$ represent streamwise 
and transverse jet turbulent intensities, $\overline{u v}$ is Reynolds shear stress, $\delta_{w}$ represents the local vorticity thickness, the turbulent kinetic energy is denoted by $k, S$ and $F$ are skewness and flatness factors, respectively, while $R_{u u}$ and $R_{v v}$ are the two-point autocorrelation functions for the streamwise and transverse velocity fluctuations, respectively.

Table 2.1 Summary of test conditions, measurement technique and flow parameters analyzed in relevant free twin jet studies

\begin{tabular}{|c|c|c|c|c|c|c|}
\hline Author & Technique & $\operatorname{Re}$ & $\begin{array}{l}\text { Nozzle } \\
\text { contour }\end{array}$ & $\begin{array}{c}\text { Nozzle } \\
\text { geometry }\end{array}$ & $S / d$ & Flow parameters \\
\hline $\begin{array}{c}\text { Miller et al. } \\
\text { [17] }\end{array}$ & HWA & 17,800 & $\mathrm{SC}$ & Plane & 6 & $\begin{array}{c}x_{m p}, x_{c p} \\
y_{0.5} \overline{u v}, \overline{u^{2}}, \overline{v^{2}}\end{array}$ \\
\hline $\begin{array}{c}\text { Lin et al. } \\
\text { [18] }\end{array}$ & HWA & 9,000 & $\mathrm{SC}$ & Plane & $\begin{array}{l}\leq \\
30\end{array}$ & $\begin{array}{c}x_{m p} \\
K_{u}, K_{s 0.5} \\
U, u_{r m s}, v_{r m s}\end{array}$ \\
\hline $\begin{array}{l}\text { Nasr et al. } \\
\text { [19] }\end{array}$ & LDA & $\begin{array}{c}8,300 \\
11,000 \\
13,750 \\
16,500 \\
19,300\end{array}$ & $\mathrm{SC}$ & Plane & $\begin{array}{c}4.2 \\
5\end{array}$ & $\begin{array}{l}P_{c} \\
K_{u} \\
\overline{u v}\end{array}$ \\
\hline $\begin{array}{c}\text { Anderson et } \\
\text { al. [20] }\end{array}$ & HWA & 6,000 & $\mathrm{SC}$ & Plane & $\begin{array}{c}9 \\
13 \\
18 . \\
25\end{array}$ & $\begin{array}{c}K_{u} \\
U_{s y m} \\
u_{r m s}, v_{r m s}\end{array}$ \\
\hline $\begin{array}{c}\text { Okamoto et } \\
\text { al. [22] }\end{array}$ & Pitot tube & 2,300 & $\mathrm{SC}$ & Round & $\begin{array}{c}5 \\
8.0 \\
6\end{array}$ & $\begin{array}{c}x_{m p}, x_{c p} \\
K_{u}, K_{s 0.5} \\
U\end{array}$ \\
\hline $\begin{array}{l}\text { Meslem et } \\
\text { al. [23] }\end{array}$ & PIV & 761 & SC & $\begin{array}{l}\text { Round } \\
\text { Cross }\end{array}$ & 2.7 & $\begin{array}{c}U_{j} \\
x_{m p}, x_{c p} \\
y_{+0.5,}, y_{-0.5}\end{array}$ \\
\hline $\begin{array}{c}\text { Harima et al } \\
{[24]}\end{array}$ & HWA & 25,000 & SC & Round & $\begin{array}{l}2 \\
4 \\
8\end{array}$ & $P_{c}, x_{c p}, U$ \\
\hline $\begin{array}{l}\text { Harima et } \\
\text { al. [25] }\end{array}$ & HWA & 25,000 & SC & Round & $\begin{array}{l}2 \\
4 \\
8\end{array}$ & $u_{r m s}, \overline{u v}$ \\
\hline $\begin{array}{l}\text { Present } \\
\text { Study }\end{array}$ & Planar PIV & 10,000 & $\begin{array}{l}\text { Sharp } \\
\text { contracti } \\
\text { on }\end{array}$ & Round & $\begin{array}{l}2.8 \\
4.1 \\
5.5 \\
7.1\end{array}$ & $\begin{array}{c}U, V, \delta_{w} \\
u_{r m s}, v_{r m s}, \overline{u v}, \\
k \text { budget } \\
S, F \\
R_{u u}, R_{v v}\end{array}$ \\
\hline
\end{tabular}


One of the early pioneers to investigate the development of twin jets is Miller et al. [17] who studied the characteristics of twin plane jets at nozzle spacing ratio of $S / d=6$ and $R e=$ 17,800. It was observed that, shortly downstream of the twin nozzles' exits, a negative gauge static pressure region is created between the jets due to the pumping effect of turbulent momentum from the jets to this central region. The negative pressure in the central region is the cause of curvature of the individual jets towards each other. In addition, the plane jets' merging process is defined by two distinct streamwise locations. The first location is the flow stagnation or merging point, which is defined as the end of the velocity re-circulation region and the onset of continuous positive velocity vectors in the central region. At the merging point, the Reynolds shear stresses that tend to accelerate the mean flow in the positive streamwise direction become equal and opposite to the static pressure gradient that tends to accelerate the mean flow in the negative streamwise direction. These opposing effects lead to a "flow stagnation" which is marked by a streamwise velocity value of zero. Based on this definition, the merging point was determined to be at $x / d=7$. The second location is the combined point, defined as the streamwise location where the jet centerlines fully merge and resemble a classical single jet flow. The combined point was determined to be at $x / d=12$. The study combined the three regions described in Section 1.2 into two regions: the converging region, which stretches from the nozzle exit up to the combined point, and the combined region which extends from the combined point onwards. In the converging region, one dimensional profiles of the streamwise mean velocity, normalized by the nozzle exit velocity, revealed comparable jet widths between the twin jets and the single jet at several streamwise locations. It should be noted that the jet width, in this study, was calculated as the distance between the inner and outer layer half-velocity widths. Profiles of the Reynolds shear stresses, on the other hand, revealed some notable differences between the asymmetrical two-peak curve classically observed for a single jet and that of twin jets. Upstream of the merging point, the profiles revealed a more 
rapid growth of the inner shear layer peak relative to the outer peak. Downstream of the merging point, however, the inner stress peak decayed rapidly, relative to the outer peak, until it completely vanished at the combined point. The study did not provide a rationale for the aforementioned behavior of the Reynolds shear stress in the inner shear layer, however, they noted that it could arise from moderate differences in the turbulent kinetic energy production and transport between the inner and outer shear layers of the twin jets. Downstream of the combined point, the mean velocity and Reynolds shear stress of the combined jet become fully developed at the same streamwise location of $x / d=23$, characterized by the self-similarity of the dimensionless profiles across the flow field, followed by the transverse Reynolds normal stress, and lastly the streamwise Reynolds normal stress at $x / d=40$. In the fully developed region, the combined jet's mean velocity was characterized by a linear spread in the streamwise distance following Eqn. $1.2 \mathrm{~m}$ while the jet centerline velocity decay was characterized by a power-law given by:

$$
\frac{U_{c l}^{2}}{U_{j}^{2}}=K\left(\frac{x-C_{0}}{d}\right)^{-c}
$$

where $K$ and $c$ are experimental constants. It was observed that the rates of the spread and decay of the combined jets in the fully developed region were identical to that of the single jet.

In another experimental work, Lin et al. [18] investigated the effect of nozzle spacing on the merging point of two plane parallel jets in the range $S / d \leq 30$ at $R e=9,000$. The converging region exhibited a depression zone, indicated by negative velocity in the symmetry plane as was described by [17]. Therefore, the merging point was defined as the streamwise location where the symmetry plane velocity values change signs to positive values. Based on this definition, the merging point was found to vary linearly with $S / d$ according to: 


$$
S / d=-18.73+2.085\left(x_{m p} / d\right)
$$

Detailed measurements of the mean velocity and turbulent statistics were then performed at $S / d=30$, and the results were compared with the single plane jet results of Gutmark et al. [26]. It was observed that, beyond the potential core region which extended to approximately $x / d=5$, the centerline velocity decays linearly at a rate of 0.428 in the converging region and at a lower rate of 0.211 in the combined region; both of which are larger than 0.184 reported for the single plane jet. The spread rates of the half-velocity width in the inner and outer shear layers within the converging region were 0.118 and 0.183 , respectively. They attributed the higher spread rate in the outer shear layer to enhanced ambient fluid entrainment in the outer layer relative to the inner layer which is confined by the two jets. In the combined region, the spread rate was 0.155 , compared to 0.102 for the single plane jet. The higher decay and spread rates reported for the twin jet compared to the single jet suggest that the twin jets experienced superior mixing with the ambient environment than the single jet. Analysis of one-dimensional profiles of the streamwise and transverse turbulent intensities and Reynolds shear stress in the outer shear layer revealed that these statistics are initially highest around the half-velocity width locations and gradually get milder and propagate towards the jet centerline. In the combined region, the streamwise turbulent intensities still maintain their maximum values around the half-velocity width but the transverse intensity peaks fully propagated to the jet centerline. Nonetheless, both turbulent intensities and Reynolds shear stress exhibit self-similar profiles in the combined region that are qualitatively similar to the self-similar single jet.

Nasr et al. [19] investigated the effects of $R e$ on the streamwise mean velocity of plane twin jets. The experiments were performed at a fixed $S / d$ of 4.25 and the $R e$ was varied from 8,300 to 19,800 . The results indicated that the distributions of the streamwise velocity were independent of $R e$ in the range 11,000 to 19,300 , indicating an independence of $R e$ at large $R e$ 
values in a similar fashion to the single jet studies in Section 2.1. Detailed measurements of the mean velocity, turbulent intensities and Reynolds shear stress were then performed at $S / d=$ 4.25 and $R e=11,000$ and the results were compared with single plane jet data obtained in the same facility and at the same $R e$ of 11,000 . The results showed that the centerline velocity of the single jet was approximately $13 \%$ higher than that of the twin jets before the combined point, but $28 \%$ lower after the combined point. The distributions of streamwise and transverse turbulent intensities across the flow field showed that their peak values in the inner shear layer were suppressed in comparison to the corresponding peak values in the outer shear layer. In contrast to the turbulent intensities, the levels of the Reynolds shear stress in the inner and outer shear layers were similar.

In a more recent work, Anderson et al. [20] performed experiments on plane twin jets at $R e \approx 6,000$ and for $S / d=9$ to 18.25 to study the effect of nozzle spacing on the merging and combined points. Following [27], they defined the merging point as the streamwise location where the streamwise turbulent intensities along the symmetry plane, normalized by the corresponding streamwise mean velocity, rapidly increases towards an asymptote. The combined point was defined as the location where the twin jets fully merged as a single jet. Both the merging and combined points were observed to increase linearly with increasing spacing ratio, which is in qualitative agreement with results presented by [18]. The asymptotic value of the streamwise turbulent intensity of the combined twin jets was 0.25 for all spacing ratios, and this value matches the value reported by Gutmark et al. [26] for a single plane jet. However, increasing the spacing ratio progressively increased the asymptotic value of the transverse turbulent intensity from the classical single jet value of 0.20 reported by [26]. The study also assessed the ability of standard k- $€$ model and Reynolds stress transport model to predict the experimental results. All models predicted a more rapid merging of the jet than the 
measured value, however, the asymptotic behavior of the Reynolds normal stresses along the jets' symmetry plane was predicted reasonably well.

The first 3D twin jet study was performed by Okamoto et al. [22]. In their investigation, pitot tubes were used to measure the mean velocity in twin round jets for $S / d=5$ and 8.06 at a fixed $R e$ of 2,300 . The merging points were found to be $x / d=8$ and 25 for $S / d=5$ and 8.06 , respectively, while the combined point were $x / d=56$ and 72 for $S / d=5$ and 8.06 , respectively. The decay rate was independent of spacing ratio, and good agreement was observed between the single jet and twin jets up to $x / d=35(S / d=5)$ and $40(S / d=8.06)$. Beyond these streamwise locations, the centerline velocities of the twin jets decayed at approximately $22 \%$ higher rate than that of the single jet due to the convergence of the jet centerlines towards the symmetry plane. The spread of the inner and outer regions of the twin jets was examined using a velocity threshold of $0.1 U_{c l}$ as opposed to the conventional $0.5 U_{c l}$. The results revealed that the spread in the inner shear layer of the twin jets agreed well with the single jet up to the locations where the symmetry plane velocity rose above zero. Beyond these locations, the inner layer spread at approximately $30 \%$ faster rate than the single jet for both spacing ratios. On the contrary, the outer shear layer of the twin jets spread at approximately $13 \%$ slower rate than the single jet.

Meslem et al. [23] compared the combined point location, spreading and entrained flow rate of twin jets issuing from round and lobed cross-shaped orifice nozzles at a fixed $S / d$ of 2.7 using a stereoscopic PIV system. The exit $R e$ was 761 for the round configuration and 741 for the cross-shaped configuration. One-dimensional profiles of the streamwise mean velocity at the round nozzles' exits collapsed with their round single jet counterpart. This, however, was not the case for the cross-shaped geometry, for which a slight contraction of the twin jet profile was noted in both the major and the minor planes of the cross in comparison with its single jet counterpart. Due to the absence of a recirculation zone in the converging region of threedimensional twin jets, the merging point was defined according to [28] as the streamwise 
location where the symmetry plane velocity reaches $10 \%$ of the jet centerline velocity. With this definition, it was shown that the converging region was absent, and the merging region actually commenced at the nozzle exit for both nozzle geometries. The combined point on the other hand, was defined as the streamwise location where the symmetry plane and jets' centerline velocities met. Based on this definition, the combined point was determined to be at $x / d=20$ and 25 for the cross twin jets and round twin jets, respectively, indicating an improved mixing in the inner region between the jets produced from the cross nozzle geometry. The stronger jet mixing of the cross jets translated into a higher spreading of the outer shear layers in comparison to the round jets. The authors defined the width of the jets' outer shear layers as the distance between one jet's outer shear layer half- velocity width and the other jet's outer shear layer half-velocity width. The rate of the outer shear layer spreading at $x / d \leq 15$ exhibited a linear behavior in both cases and was determined to be 0.103 and 0.152 for the round twin jets and cross twin jets, respectively. The results were supported by determining the entrained flow rate of the cross nozzle to be approximately 3 times higher than that of the round orifice.

In a more recent study, Harima et al. [24,25] investigated the effects of nozzle spacing on the mean and turbulent statistics of twin round jets by the examining the effect of three spacing ratios, $S / d=2,4$ and 8 at a fixed $R e$ of 25,000 . The measured potential core length was $4 d$ for all the spacing ratios, however, the merging and combined points were located at $x / d=2,9$ and 28 and $x / d=25,52$ and 90 for $S / d=2,4$ and 8, respectively, suggesting a linear increase with nozzle spacing, similar to plane twin jets $[18,20]$. Moreover, the evolution of streamwise turbulent intensity along the jets' symmetry plane, normalized by the exit velocity, revealed maximum peaks at $x / d=7.7,15.8$ and 27.8 for $S / d=2,4$ and 8 , respectively, followed by a monotonic drop in intensity. The evolution of streamwise mean velocity along the symmetry plane, normalized by the jets' exit velocity, revealed a qualitatively similar behavior to the turbulent intensities, where the mean velocity exhibited a peak at $x / d=12,22$ and 40 for $S / d$ 
$=2,4$ and 8 , respectively, followed by a monotonic drop. These results suggested that the earlier merging of the jets at lower spacing ratios leads to an earlier rise and higher peak levels of mean velocity and turbulent intensity along the symmetry plane. As shown, however, the turbulent intensity peaks occurred at earlier streamwise distances than their mean velocity counterparts, indicating that the effect of the jets' interference appears faster in the streamwise turbulence intensities than the streamwise mean velocity. Unlike the distributions of the streamwise turbulent intensities along the symmetry plane, the peak values of the streamwise turbulent intensities along the jet centerline were independent of nozzle spacing but their corresponding streamwise locations slightly decreased with increasing spacing, i.e., $x / d=9.1$, 8.3 and 7.6 respectively for $S / d=2,4$ and 8 . Similar to the findings of [17] for plane jets, it was observed that downstream of the merging point, the levels of the Reynolds shear stress were suppressed in the inner shear layer for $S / d=2$ and 4 owing to the interference between twin jets, but the suppression of the Reynolds shear stress in the inner layer was absent for $S / d$ $=8$.

\subsection{Summary of Literature}

It is evident from the literature review presented above that although the fundamental features of twin jets have been studied by many researchers, most of these investigations have focused exclusively on the mean flow and the second order moments. However, investigation of higher order turbulent statistics and large scale turbulent structures are also necessary to develop a deeper understanding of the flow field and help interpret the trends observed in the mean flow.

The literature also shows that the mixing performance, measured using parameters like potential core length, decay and spread rates, is influenced by jets' interaction [17-19,22], but discrepancies exist regarding whether these parameters are enhanced or suppressed upstream 
and downstream the combined point, relative to single jet flow. The jets' interaction is also shown to generally suppress the Reynolds stresses profile peaks in the jets' inner shear layers relative to the outer stress peaks $[17,19,25]$. However, a couple of studies have reported the enhancement of Reynolds stress peaks in the jets' inner layer upstream of the merging point before the suppression commences $[17,25]$, but no explanation to this phenomenon and/or its relation to the turbulent kinetic energy transport terms has been provided.

In addition, all studies who compare the twin jet flow development with a free single jet have used single jet results from other authors [18,20,22]. Even though it is well known that the mean flow and higher order turbulent statistics could be facility dependent, no study has yet systematically compared the twin jet flows with a free single jet study that is performed in the same test facility and under the same test conditions.

Lastly, aside from [20] for twin plane jets, no study has reported comprehensive benchmark experimental datasets to help verify and optimize the numerical codes used in numerical simulation of twin jets.

\subsection{Scope of Research}

To help address the aforementioned knowledge gap, a planar PIV system is employed to investigate the mean flow and turbulent characteristics of twin jets issued from round sharp contraction nozzles at $R e=10,000$. Four spacing ratios are investigated and the results are compared and contrasted with a free single jet study that was performed in the same test facility and under the same test condition. The salient features of the flow are examined by employing contours plots and one-dimensional profiles of mean velocity and Reynolds stresses across the flow field. The mixing characteristics are examined using the potential core length as well as the centerline velocity decay and spread rates. The interaction of the jets in various flow regions is also analyzed by tracking the evolution of mean velocity, vorticity thickness and turbulent 
intensities along the centerline and symmetry plane. Large-scale anisotropy is investigated using the ratio of Reynolds normal stresses, and skewness and flatness factors are investigated along the jet centerline as well as the symmetry plane to shed light on the symmetry and flatness of the probability density function. Lastly, two-point correlation analysis is employed to examine the effects of nozzle spacing on the large-scale turbulent structures in the various flow regions. 


\section{CHAPTER 3}

\section{EXPERIMENTAL SETUP AND MEASURMENT PROCEEDURE}

In this chapter, an overview of the air chamber test facility, the PIV system and the measurement procedure are reported. This is followed by a summary of the test conditions and estimated measurement uncertainties. The uncertainty analysis undertaken in this study is discussed in detail in Appendix B.

\subsection{Experimental Setup}

A schematic and depiction of the air chamber test facility used in this study is shown in

Fig. 3.1. The air chamber is $1100 \mathrm{~mm}$ long, $734 \mathrm{~mm}$ wide and $534 \mathrm{~mm}$ deep, and the top and side walls are made of transparent acrylic sheet to facilitate optical access. The acrylic sheets used to fabricate the test facility are $24.5 \mathrm{~mm}$ thick. The $x$ coordinate is aligned with the streamwise direction, while the $y$ coordinate is in the normal direction; $x=0$ is at the nozzle exit and $y=0$ is at the symmetry plane between the two nozzle exits. Several perforated stainless steel plates and a polycarbonate honeycomb are fitted upstream of the test section to break down the large-scale turbulent structures and homogenize the incoming flow. The lab air, at about $600 \mathrm{kPa}$ and ambient temperature of $20^{\circ} \mathrm{C}$, is introduced through a LaVision aerosol generator, then through the nozzles and subsequently into the air chamber. The air temperature, pressure and flow rate were monitored to ensure that they remain constant in all experiments. The aerosol generator was also used to produce small oil droplets (mean diameter of $1 \mu \mathrm{m}$ ) as seeding particles for the PIV measurements, which is described in more detail in Section 3.2. The amount of aerosols introduced into the supply air and through the nozzles into the test section was controlled by two bypasses. The outlet section consists of a fan driven filtration system that draws the air remaining in the air chamber through filters before exhausting into the room. 


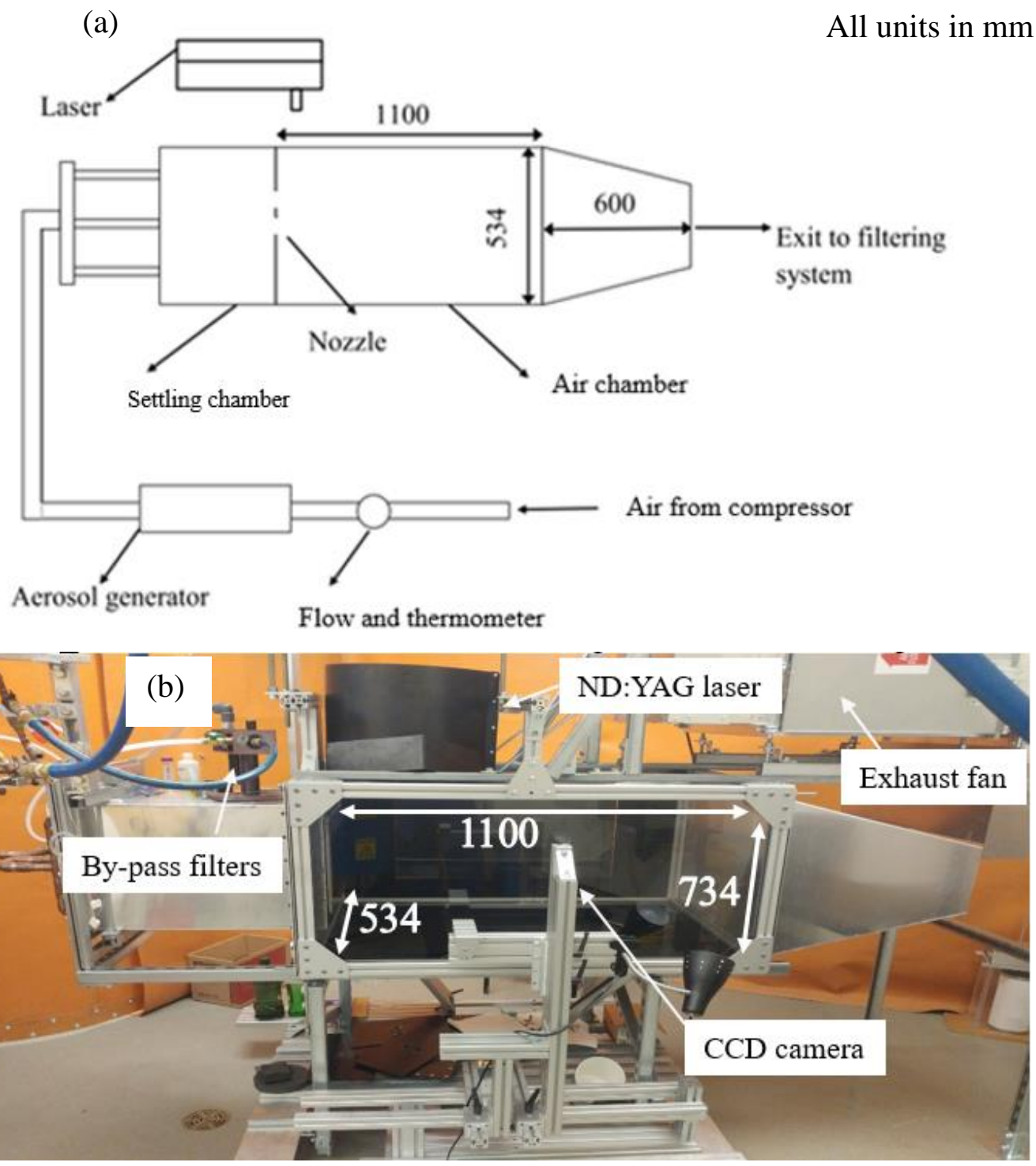

Figure 3.1: (a) Schematic and (b) picture of air chamber test facility

Two identical round nozzles, adopted from $[9,29,30]$, were used in the present study. As shown in Fig. 3.2, the nozzle have a sharp linear contraction with a length $(t)$ of $6 \mathrm{~mm}$ and outlet diameter of $d=9 \mathrm{~mm}$. The contraction section is preceded by a straight section of diameter, $D=18 \mathrm{~mm}$ and length, $L=20 \mathrm{~mm}$, producing an area ratio of 4 . This particular nozzle was shown to have mixing characteristics that are intermediate to those reported for OP and SC nozzles [9]. Although orifice nozzles appear to be superior from a mixing perspective, Price Industries' motivation behind testing the performance of the sharp contraction nozzle is to 
mitigate possible 'cavitation' phenomenon encountered by OP nozzles, as described in Section 2.1. The acoustic advantage of the sharp contraction nozzle was not verified in this study, however, its use was recommended by Price Industries.

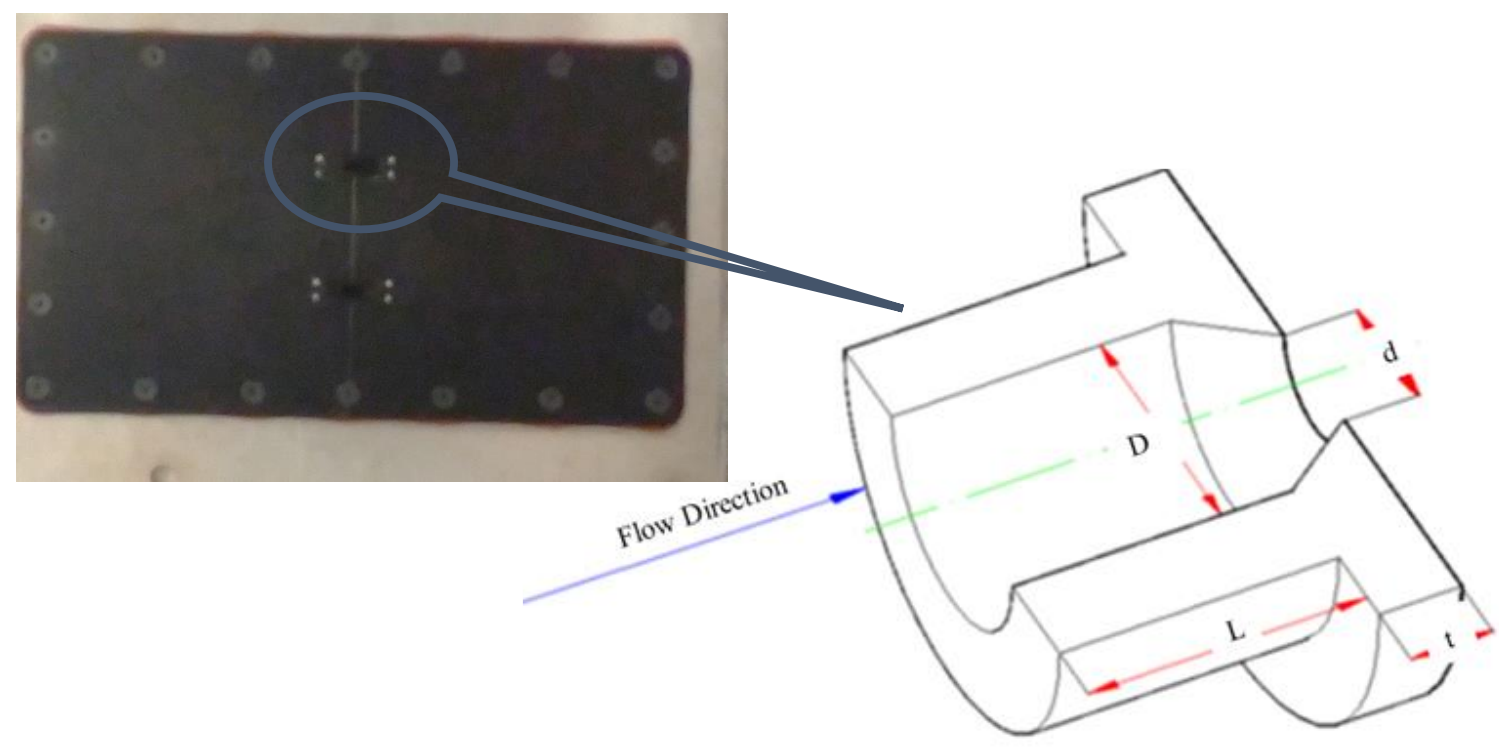

Figure 3.2: Test round nozzle with a modified contraction

\subsection{PIV System and Measurement Procedure}

The velocity measurements were performed using a planar PIV system. The basic principle of a PIV is as follows: the flow is seeded with small light scattering particles, known as seeding particles that are presumed to follow the flow faithfully. The flow field is then illuminated by two pulses of laser that are separated by a time delay, $\Delta t$. Each pulse represents an image, and therefore, two successive images are recorded per pulse cycle. In the post processing stage, the images are divided into grids called interrogation areas. For each interrogation area, a correlation based algorithm is applied to statistically determine the local displacement vector $\Delta s$ of particles between the first and second image. Averaging over multiple particle pairs within an interrogation area makes correlation algorithms remarkably noise-tolerant and robust in comparison to tracking individual particles. There are two types of correlation algorithms: auto-correlation, in which the same image is correlated with itself with a time shift, and cross- 
correlation, where two independent images with a time shift are correlated. Auto-correlation has the ability to measure the magnitude of the particle displacement but not its direction. Cross-correlation, on the other hand, is able to measure the magnitude and direction of the particle displacement as well as zero displacements. Subsequently, the velocity, $V$, for a particular interrogation area is obtained from the expression, $V=\Delta s / \Delta t$. A velocity vector map over the whole target area is obtained by repeating the correlation for each interrogation area over the two images captured. Since the entire flow field can be analyzed at once, the PIV provides simultaneous whole field measurement. The following sections provide an overview of the various PIV components selected for the present study.

\subsubsection{Seeding Particles}

In the present study, the flow was seeded with $1 \mu \mathrm{m}$ oil droplets that were generated by the aerosol generator. These seeding particles were chosen because they are large enough to scatter sufficient light to be detected by the digital camera and small enough to follow the flow faithfully. According to [31] and [32], the scattering cross section (which is a convenient measure of light scattering capability) of $1 \mu \mathrm{m}$ particle diameters is approximately $10^{-12} \mathrm{~m}^{2}$, which is within the recommended scattering cross section range for sufficient light scatter. Moreover, the flow tracking capability of the particle is measured using the particle's settling velocity and response time. According to [32], the particle's settling velocity $U_{s}$ is calculated as:

$$
U_{s}=\frac{d_{p}^{2}\left(\rho_{p}-\rho_{f}\right)}{18 v_{f}}
$$

where $d_{p}$ is the particle object diameter, $\rho_{p}$ is particle density, $\rho_{f}$ is fluid density and $v_{f}$ is fluid kinematic viscosity. For the present study, $U_{s}$ was calculated to be $4.68 \times 10^{-5} \mathrm{~m} / \mathrm{s}$ which is up to five orders of magnitude smaller than the streamwise mean velocities measured. In 
addition, the particle's time response is a measure of the tendency of particle to attain velocity equilibrium with the fluid. According to [33], the particle's time response $t_{s}$ is calculated as

$$
t_{s}=\frac{d_{p}^{2} \rho_{p}}{18 \mu_{f}}
$$

where $\mu_{f}$ is fluid dynamic viscosity. For the present study, $t_{s}$ was calculated to be $4.78 \times 10^{-6} \mathrm{~s}$, which is very small compared to the flow time scale in this study. Therefore the combination of settling velocity and time response results clearly demonstrate that the size and type of seeding particles chosen satisfy the flow tracking ability of the seeding particles.

\subsubsection{Laser Source}

For PIV measurements, a high intensity pulse laser is required to freeze the motion of the particles during image capturing. The laser intensity must also ensure that the camera can capture sufficient sideward light scattered by the particles. A neodymium-yttrium-aluminumgarnet (Nd:YAG) double-pulsed laser was used as it provides monochromatic light with high intensity illumination. The laser has a thickness of $1 \mathrm{~mm}$ and a maximum energy of $120 \mathrm{~mJ}$ per pulse at $532 \mathrm{~nm}$ wavelength. The laser pulse separation time $\Delta t$ was determined based on the quarter displacement rule $[34,35]$ so that the seeding particles move no more than a quarter of the size of the interrogation area to ensure a good signal-to-noise ratio and minimize loss of particle pairs. $\Delta t$, therefore, was determined using the following expression:

$$
\Delta t=\frac{I A x d_{\text {pitch }}}{4 M U_{\max }}
$$

where IA is the interrogation area size ( $32 \times 32$ pix), $d_{\text {pitch }}$ is the pixel pitch ( 7.4 pix), $M$ is the magnification factor (20.48 pix/mm), and $U_{\max }$ is the maximum velocity of the flow $(16.8 \mathrm{~m} / \mathrm{s})$. Consequently, $\Delta t$ was determined to be $23 \mu \mathrm{s}$. 


\subsubsection{Recording Medium}

The light scattered from the seeding particles was captured by a 12-bit charge-coupled device $(C C D)$ camera with a resolution of 2048 pixel $\times 2048$ pixel and a pixel pitch of $7.4 \mu \mathrm{m}$ coupled to a $60 \mathrm{~mm}$ AF Micro Nikkor lens. This CCD camera uses high-performance scan interline CCD chips. Each chip consists of an equal number of photosensitive cells and storage cells. After the first laser pulse is acquired, the first image is acquired and transferred from the photosensitive cells to the storage cells. When the second laser pulse is triggered, the photosensitive cells are available to store the second image. Both images are then transferred sequentially from the camera to the computer for storage. This allows the exposure time to be reduced to less than $1 \mu \mathrm{s}$. The particle image diameter $d_{\text {image }}$ in pixels was calculated according to [33] as follows:

$$
d_{\text {image }} \approx \sqrt{d_{p}^{2} M^{2}+\left(\left(2.44(1+M) f_{\#}\right) \lambda\right)^{2}}
$$

where $f_{\#}$ is the focal number of the lens (4) and $\lambda$ is the wavelength of Nd:YAG $(532 \mathrm{~nm})$. Consequently, $d_{\text {image }}$ was determined to be approximately 0.81 pixels. A particle image diameter of 2 pixels is recommended by [33] as the ideal particle image dimeter, because if $d_{\text {image }}$ becomes too small, the likelihood of peak locking increases, where the correlation peaks are biased towards integer pixel values as opposed to obtaining accuracies within fractions of a pixel and allowing for sub-pixel displacement interpolation. However, in practical PIV experiments, this is not often possible, and so the particle image diameter of the present study suggests possible peak locking but the effect was minimized by using a Gausssian low-pass filter. 


\subsubsection{PIV Post Processing}

The instantaneous digital images were post-processed using adaptive correlation option by DynamicStudio v4.1 [36]. Adaptive correlation is an advanced type of the standard crosscorrelation that uses a multi-pass fast Fourier transform (FFT) algorithm with a onedimensional Gaussian peak-fitting function to determine the average particle displacement within the interrogation area. A moving average validation was used during image processing to reject outlier velocity vectors that deviate too much from their neighbors. The IA was set to 32 pixels $\times 32$ pixels with $50 \%$ overlap in both $x$ and $y$ directions. With an IA size of 32 pixels $\times 32$ pixels, the maximum particle displacement in the streamwise direction was 8 pixels with a dynamic range of 80 , in order to comply with the quarter displacement rule described in Section 3.2.2.

\subsection{Experimental Test Conditions}

The experiments were performed for spacing ratios, $S / d=2.8,4.1,5.5$ and 7.1 at $R e=$ 10,000. The rationale for the choice of this particular $R e$ was to facilitate comparison of the present study with a previous single jet study [9] performed as part of this collaborative research with Price in the same test facility that showed that statistical properties reach $R e$ independency at $R e \geq 10,000$. A subsequent investigation of $R e$ effects on twin round jets produced from these nozzles at a fixed $S / d$ of 2.8 also demonstrated that the mean flow and turbulent characteristics were independent of Reynolds number for $R e \geq 10,000$ [37].

The measurements were obtained in 4 or 5 overlapping planes that extended from the jet exit up to $x=40.5 d(S / d=2.8,4.1)$ and $x=50.5 d(S / d=5.5,7.1)$ to ensure that the converging, merging and combined regions were captured. Based on preliminary statistical convergence tests (see Appendix A for details), an ensemble size of 7500 statistically independent image pairs were acquired at a sampling rate of $2.5 \mathrm{~Hz}$ in each measurement plane. 


\subsection{Uncertainty Estimates}

Measurement uncertainty analysis was performed following the AIAA standard as explained by [38] and [39]. In general, the accuracy of PIV measurements is limited by the accuracy of the sub-pixel interpolation of the displacement correlation peak. Other sources of error include particle response to the flow motion, light sheet positioning, light pulse timing and the size of interrogation area which determines its spatial resolution. On basis of the size of interrogation area and the number of instantaneous vector maps used to calculate the mean velocity and turbulent quantities, the estimated uncertainty for the mean velocity was determined to be approximately $\pm 2.45 \%$ of its peak value. The uncertainties of turbulence intensities and the Reynolds shear stresses were approximately $\pm 7 \%$ and $\pm 10 \%$ of their peak values, respectively. The uncertainty in third and fourth order moments is of the order of $\pm 15 \%$ of their peak values. Detailed uncertainty analyses for this study are presented in Appendix B. 


\section{CHAPTER 4}

\section{RESULTS AND DISCUSSION}

This chapter presents and discusses the effects of nozzle spacing ratio on the mean and turbulent characteristics of the twin round jets examined in this study. The discussion is divided into five parts. The first section focuses on the effects of nozzle spacing on the mixing characteristics which are examined using the evolution of the mean flow along the jets' centerline and symmetry plane, the merging and combined points, as well as the decay and spread rates. The second section discusses Reynolds stresses and kinetic energy budget, as well as the evolution of turbulent intensities along the centerline and symmetry plane. The ratio of the Reynolds stresses along the centerline is also investigated to examine large-scale anisotropy. The third section focuses on the distributions of skewness and flatness factor along the centerline and symmetry plane to examine the asymmetry and intermittency of the velocity fluctuations. The fourth section presents one-dimensional profiles of the mean velocity and Reynolds normal and shear stresses to examine their evolution across the shear layers at different flow regions. In the last section, two-point correlation analysis is employed to investigate the spatial coherence of large-scale vortical structures at select flow regions.

\subsection{Mean Flow}

Contour plots of the streamwise and transverse mean velocity are shown in Fig. 4.1 to examine how the basic mean flow patterns vary with nozzle spacing. In these and subsequent contour plots, the nozzle diameter, $d$, and the maximum streamwise mean velocity, $U_{\max }$, are adopted as the normalizing length and velocity scales, respectively. 
$\begin{array}{llllllllllll}U^{*}: & 0.1 & 0.2 & 0.3 & 0.4 & 0.5 & 0.6 & 0.7 & 0.8 & 0.9 & 1\end{array}$

(a)

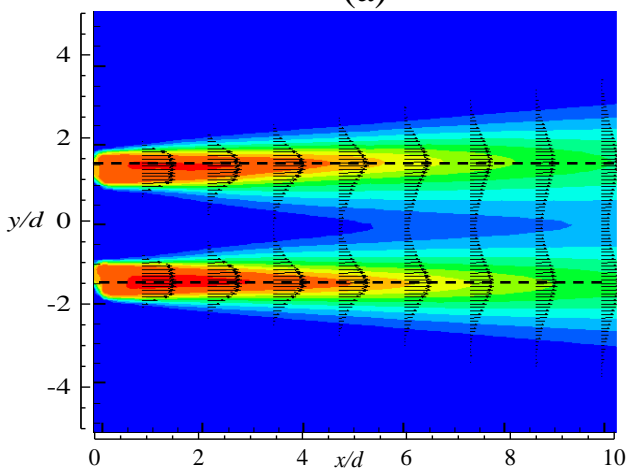

(c)

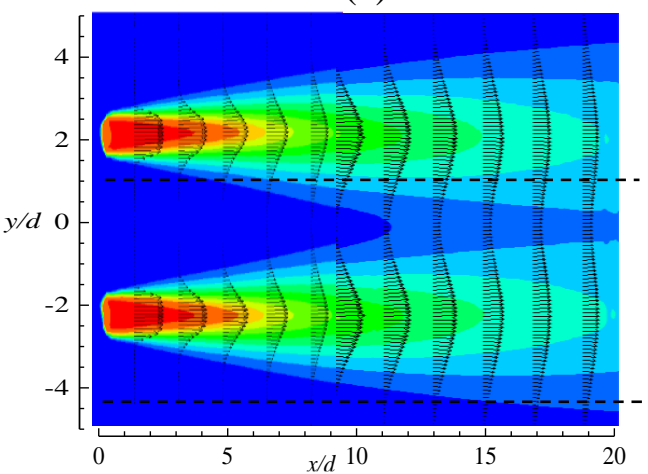

(e)

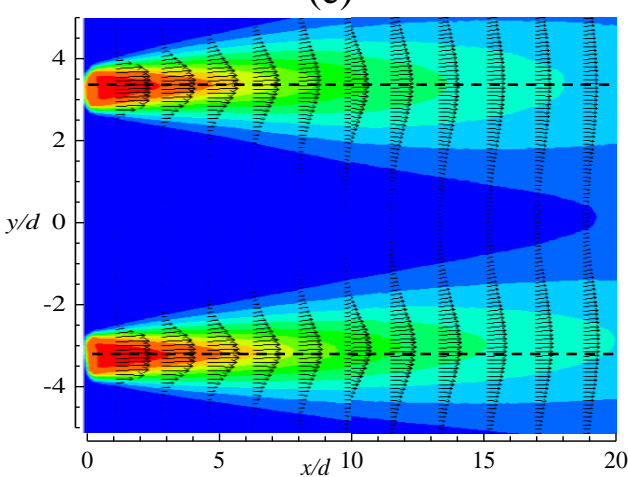

(g)



$\begin{array}{llllllll}V^{*}: & -0.02 & -0.015 & -0.01 & 0 & 0.01 & 0.015 & 0.02\end{array}$

(b)

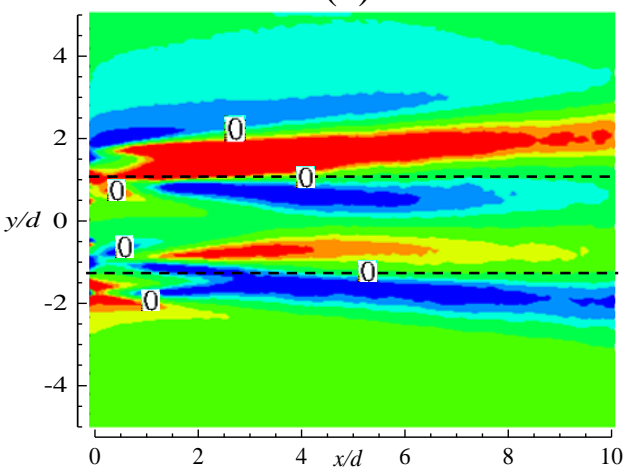

(d)

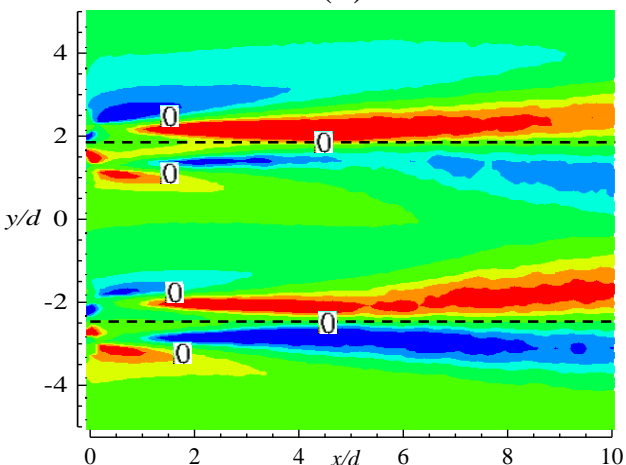

(f)

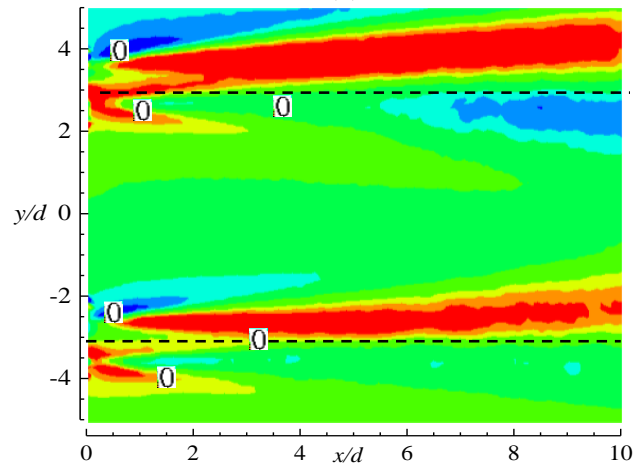

(h)

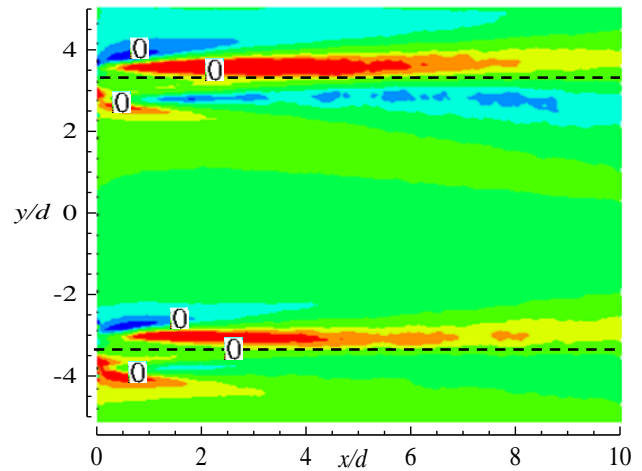

Figure 4.1: Contours of streamwise and transverse mean velocity for $S / d=2.8$ (a, b ), 4.2 (c, d ) and $5.5(\mathrm{e}, \mathrm{f})$ and 7.1 (g, h ) 
The topology of the streamwise mean velocity ( Figure 4.1a, 4.1c, 4.1e and 4.1g) provides an indication that the streamwise velocity initially increased from the exit value reaching a peak value, $U_{\max }$, at $x \approx 0.5 d$ from the nozzle exit, irrespective of spacing ratio. This phenomenon, which has been reported in previous free jets issued from OP nozzles $[9,10,15]$, is attributed to the vena contracta effect caused by the abrupt contraction of the jet. The two jets, which preserve their distinct identity initially, interact and eventually merge on the symmetry plane after a certain distance from the exit plane. The velocity magnitudes decrease in both streamwise and transverse directions as the individual jets spread outwards into the unconfined ambient fluid, and inward into the confined region between the two jets. The individual jets are remarkably similar, and the effect of reducing nozzle spacing is to enhance the mutual interaction between the inner layers of the two jets and promote an earlier merging.

The transverse mean velocity (Figure 4.1b, 4.1d, 4.1f and 4.1h), on the other hand, shows an anti-symmetrical flow pattern where the velocity across an individual jet is opposite in sign to its twin counterpart. It is observed that the dominant mechanism in the immediate vicinity of the nozzle exit is entrainment of the ambient fluid into the core jet. In this region, the outward expansion of the jets is limited, and their centerline velocities do not decay appreciably. In the inner shear layers, entrainment of fluid between the jets into the core jets, represented by positive transverse velocity for the upper jet and negative velocity for the lower jet, intensifies at a larger spacing ratio, indicated by the larger magnitude of $V^{*}$. Further downstream, the jets expand towards the outer ambient fluid, indicated by positive and negative velocities, respectively, for the upper and lower jets, and inwards, indicated by negative and positive velocities for the upper and lower jets, respectively. The enhanced magnitude of $V^{*}$ in the inner shear layers at lower spacing ratios is an indication that a reduction in nozzle spacing leads to a more intense interaction between the jets. 
The effect of nozzle spacing on the twin jets' mixing can be examined by investigating the evolution of the mean flow along the jets' centerline and symmetry plane. The streamwise mean velocity along the centerline of the lower jet and the symmetry plane are shown in Fig. 4.2a. The centerline velocity of the round single jet performed by [9] in the same facility and similar Reynolds number are shown in this figure for comparison. The mean velocities are normalized by $U_{\max }$ while streamwise distance, measured from jet exit, is normalized by $d$. Note that only the lower jet centerline velocity is included in the figure due to the symmetric pattern of the twin jets observed in Fig. 4.1.
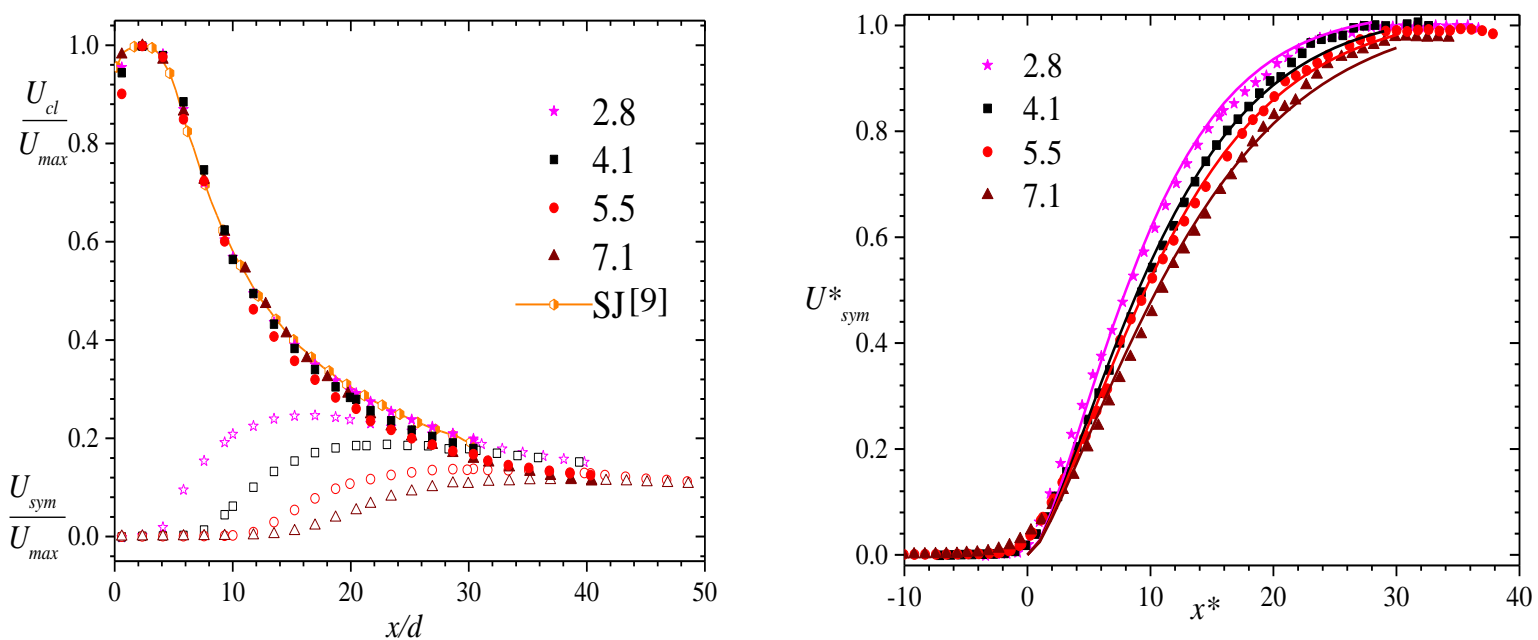

Figure 4.2: (a) Symmetry plane and jet centerline streamwise velocity downstream evolution, (b) Symmetry plane streamwise velocity normalized by $U_{c l}$ and $x_{m p}$

Along the centerline, there is an initial increase in the mean velocity (as observed in Fig. 4.1), followed by a region of near constant $U_{c l}$ and then a monotonic decrease with streamwise distance. The region where $U_{c l}$ is constant is known as the potential core. For the twin jets, the mean velocities along the centerline are independent of nozzle spacing, and are also in good agreement with the single jet data. Along the symmetry plane, the streamwise mean velocity is initially zero, followed by a rise to a maximum value where it remains invariant with streamwise distance until it merges with the centerline velocity. Unlike the 
velocity distribution along the centerline, significant effects of nozzle spacing are clearly evident along the symmetry plane. For example, more rapid rise and higher peak values are observed at lower spacing ratios. The values of the dimensionless maximum mean velocity along the symmetry plane $\left(U^{*}{ }_{s y m}\right)_{\max }$ and their corresponding streamwise location $(x / d \mid$ $\left(U^{*}\right.$ sym)max $)$ for the various spacing ratios are summarized in

Table 4.1.

Table 4.1: Streamwise mean velocity peak values and locations along the symmetry plane

\begin{tabular}{ccc}
\hline$S / d$ & $\left(\boldsymbol{U}_{\text {sym }}\right)_{\max }$ & $\boldsymbol{x} / \boldsymbol{d}_{\mid\left(\boldsymbol{U}_{\text {sym }}\right) \max }$ \\
\hline 2.8 & 0.25 & 16.1 \\
\hline 4.1 & 0.19 & 23.6 \\
\hline 5.5 & 0.14 & 30.6 \\
\hline 7.1 & 0.12 & 40.0 \\
\hline
\end{tabular}

Compared to the smallest spacing $(S / d=2.8)$, the peak values for $S / d=4.1,5.5$ and 7.1 are reduced by $24 \%, 44 \%$ and $52 \%$, and their respective streamwise locations from the jet exit are $47 \%, 90 \%$ and $148 \%$ longer. These results are in qualitative agreement with the trend reported by Harima et al. [24] for round twin jets at similar spacing ratios but at a relatively higher Reynolds number.

Fig. $4.2 \mathrm{~b}$ shows the distributions of $U^{*}$ sym normalized by $U_{c l}$, where the streamwise distance is measured relative to the merging point, $x_{m p}$, and normalized by $d$. The plots show a much better collapse (compared to Fig. 4.2a), demonstrating that the scaling adopted herein is more appropriate than $U_{\max }$. The profiles in the region $0 \leq x^{*} \leq 30$ (representing the merging region) follow a modified exponential-Gaussian function of the form [40]:

$$
U_{\text {sym }}^{*}=\frac{1}{A+B \exp \left(\frac{-x^{* 2}}{C+E x^{*}}\right)}-D
$$


where $x^{*}=\left(x-x_{m p}\right) / d$. The function fits followed the data sets very well with $R^{2}$ value of 0.98-.0.99 with the fitting coefficients summarized in Table 4.2:

Table 4.2: Summary of fitting coefficients for streamwise mean velocity scaling along the symmetry plane according to Eqn. 4.1

\begin{tabular}{|c|c|c|c|c|c|}
\hline$S / d$ & A & B & C & D & $\mathbf{E}$ \\
\hline 2.8 & \multirow{4}{*}{0.492} & \multirow{4}{*}{0.508} & \multirow{4}{*}{12} & \multirow{4}{*}{1} & 6 \\
\hline 4.1 & & & & & 7.1 \\
\hline 5.5 & & & & & 7.7 \\
\hline 7.1 & & & & & 8.8 \\
\hline
\end{tabular}

Following [25], the potential core length, $P_{c}$, was defined as the streamwise distance from the jet exit plane to the point where $U_{c l}=0.98 U_{\max }$. The potential core lengths for all the nozzle spacing ratios are within $P_{c} / d=4.1 \pm 0.1$. These values are also in good agreement with $P_{c} / d$ $=3.9$ reported for single round jets $[9,23]$ and $P_{c} / d=4.0$ for twin plane and round jets $[19,24]$. These results imply that, near the nozzle exit, the twin jets are isolated from each other and behave like a single jet, leading to a potential core length that is independent of nozzle spacing.

Consideration is now turned to the merging and combined points. For plane jets, the merging point is defined as the end of the recirculation region between the jets. However, due to the absence of a recirculation region in round twin jets, an alternate method was proposed by $[23,28,41]$ which defined the merging point as the streamwise location where $U_{s y m}=0.1 U_{c l}$. Okamoto et al. [22] also argued that the initial rise of $U_{\text {sym }}$ above zero is indicative of the onset of inner shear layer mixing. Thus, the merging point in the present study is defined as the streamwise location where $U_{\text {sym }}$ rises above zero. Since the dynamic velocity range (ratio of maximum resolvable velocity to minimum resolvable velocity) in the present study is $80, x_{m p}$ was determined as the streamwise location where $U_{s y m}=0.015 U_{\max }$, which is slightly above the theoretical minimum resolvable $U_{s y m}=0.0125 U_{\max }$. A sensitivity analysis was performed by comparing these values to those obtained from, say, $U_{s y m}=0.01 U_{\max }$ and $0.02 U_{\max }$. Variations were observed, but they were within $\pm 0.2 d$, which are within measurement 
uncertainties for the potential core length. When compared with values estimated from $U_{\text {sym }}=$ $0.1 U_{c l}$, the maximum difference between the two methods was $0.3 d$, which is also comparable to measurement uncertainty. The combined point, $x_{c p}$, was determined as the streamwise location where $U_{c l}$ and $U_{\text {sym }}$ coincide in Fig. 4.2a. As shown in Fig. 4.3, the merging and combined points for the present and previous studies increase linearly with spacing ratio.
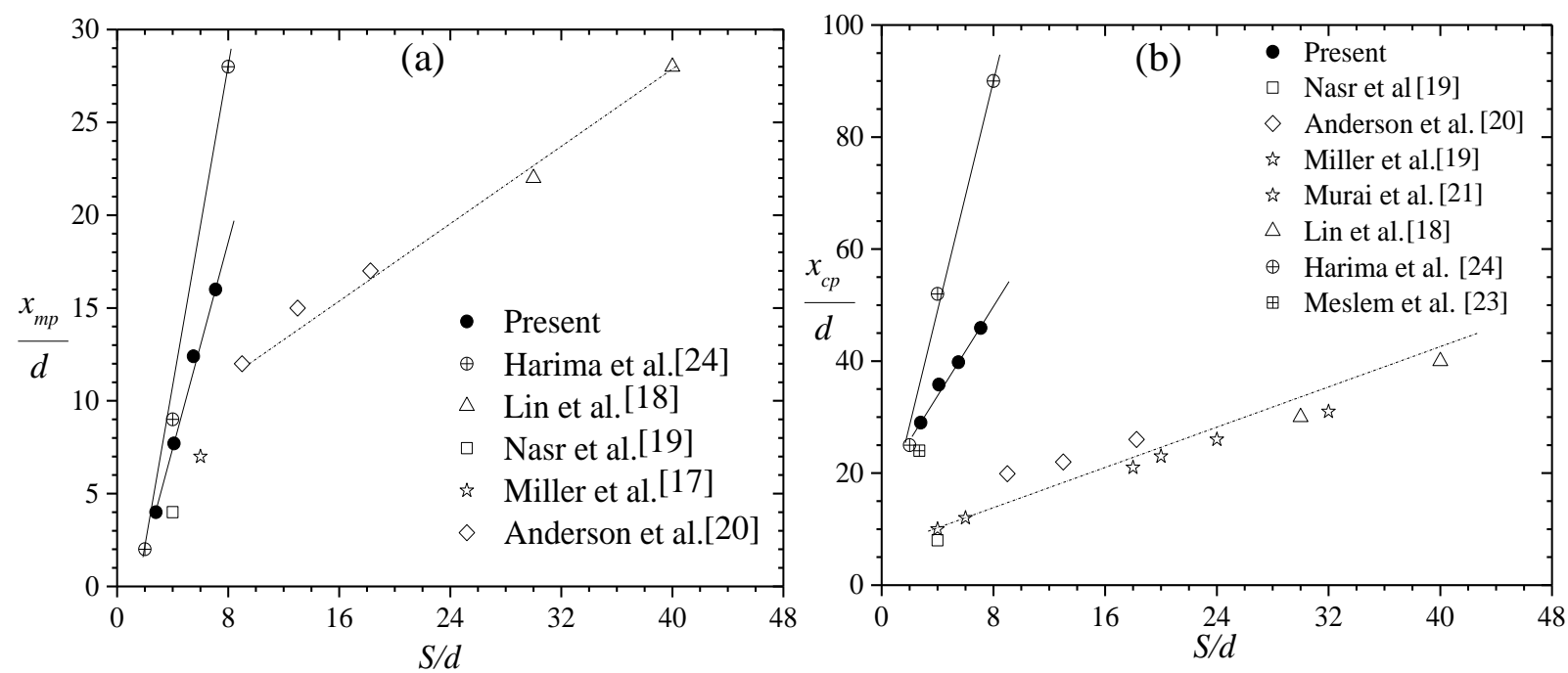

Figure 4.3: (a) Merging point, (b) combined point locations for present and previous twin jet studies

However, the merging and combined points as well as slopes vary considerably from one study to the other, which is a clear indication that the mutual interaction between the inner shear layers of the jets are strongly dependent on initial and boundary conditions. For example, the rates at which $x_{m p}$ and $x_{c p}$ for the twin round jets issued from SC nozzles [24] (cross symbols) increased with nozzle spacing are 1.5 and 2.8 times lower than the corresponding values reported in the present study (solid symbols). Moreover, the rates at which $x_{m p}$ and $x_{c p}$ increased with nozzle spacing for the present round twin jets are approximately 1.2 and 5.6 times, respectively, lower than those of plane twin jets (open symbols). 
To investigate the effects of nozzle spacing on the velocity decay, $U_{\max } / U_{c l}$ is plotted against $x / d$, as shown in Fig. 4.4. The datasets show a linear increase beyond the potential core but with different decay rates $K_{u}$ in different streamwise regions.

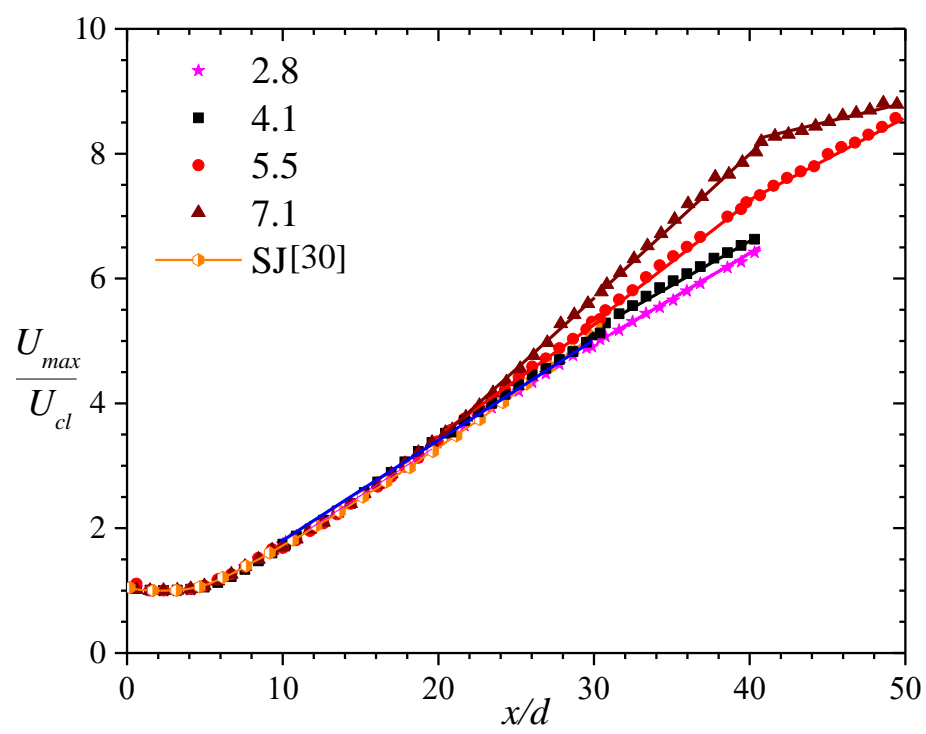

Figure. 4.4: Streamwise evolution of jet centerline velocity normalized by the maximum exit velocity

The decay rates are summarized in Table 4.3 for the twin jets and the reference single jet [30]. In the region $5 \leq x / d \leq x_{c p}, S / d=2.8$ and 4.1 have similar decay rates, however, the rate increased by approximately $11 \%$ for $S / d=5.5$ and $23 \%$ at $S / d=7.1$, indicating that a larger spacing ratio experiences enhanced fluid entrainment into inner shear layers which results in an expedited decay of the core jet velocity. In the combined region, the decay rates are reduced in comparison to values obtained upstream of the combined point by $18 \%, 35 \%, 33 \%$ and $80 \%$ for $S / d=2.8,4.1,5.5$ and 7.1, respectively. A similar observation was reported by [19] that showed that while the centerline velocity of the twin plane jets decayed at a higher rate than the single jet before $x_{c p}$, their decay rate was considerably lower in the combined region. 
Table 4.3 Summary of jet centerline decay rates before and after the combined point

\begin{tabular}{ccc}
\hline S/d & \multicolumn{2}{c}{$\boldsymbol{K}_{\boldsymbol{u}}$} \\
\cline { 2 - 3 } & $\boldsymbol{x} / \boldsymbol{d} \leq \boldsymbol{x}_{\boldsymbol{c} p}$ & $\boldsymbol{x} / \boldsymbol{d} \geq \boldsymbol{x}_{\boldsymbol{c} p}$ \\
\hline 2.8 & 0.17 & 0.14 \\
\hline 4.1 & 0.17 & 0.11 \\
\hline 5.5 & 0.19 & 0.14 \\
\hline 7.1 & 0.21 & 0.05 \\
\hline SJ [30] & \multicolumn{2}{c}{0.17} \\
\end{tabular}

Fig. 4.5 shows the half-velocity width in the outer and inner shear layers, respectively, measured from the jet centerline, while the spread rates, $\left(K_{s+0.5}, K_{s-0.5}\right)$ evaluated by fitting a linear curve to the datasets are summarized in Table 4.4.
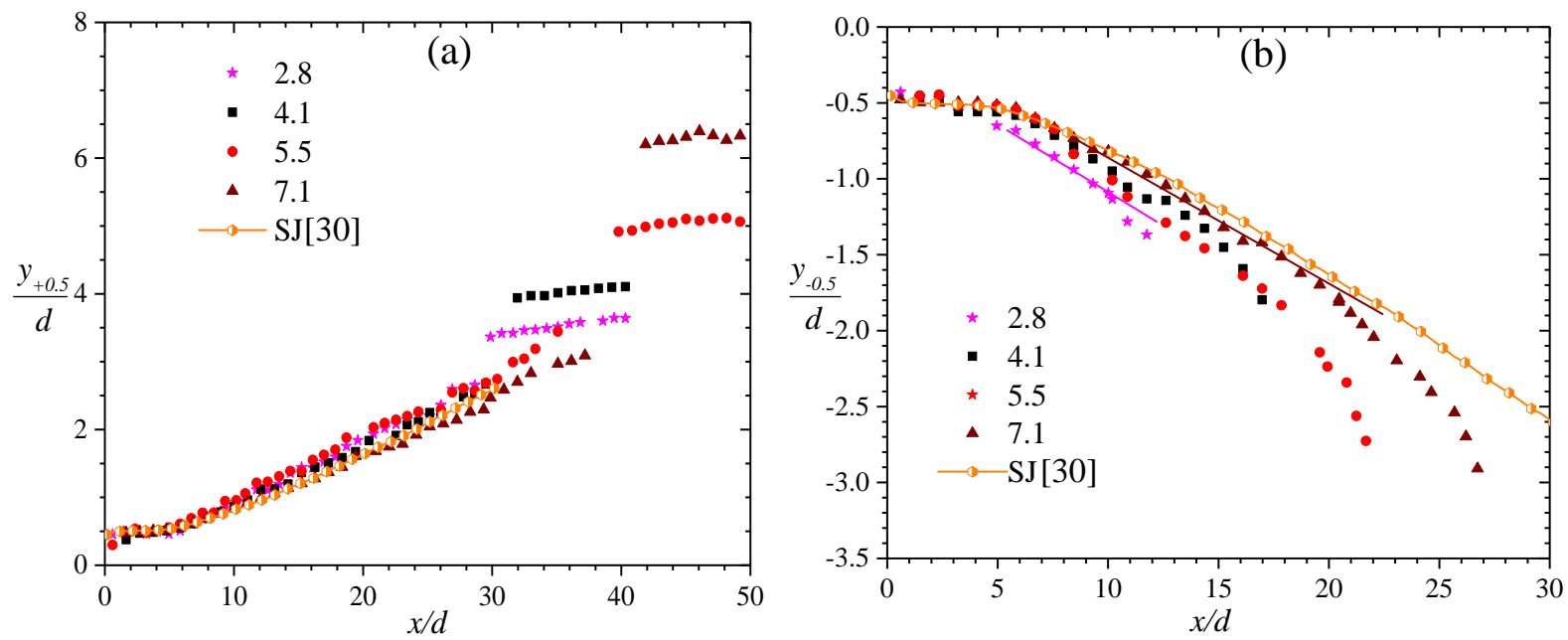

Figure 4.5: (a) Outer shear layer half-velocity spread (b) inner shear layer half-velocity spread 
Table 4.4: Summary of half-velocity width spread rates along the jet's outer and inner shear layers

\begin{tabular}{ccc}
\hline S/d & $\boldsymbol{K}_{\boldsymbol{s}+0.5}$ & $\boldsymbol{K}_{\boldsymbol{s}-0.5}$ \\
\hline 2.8 & 0.09 & 0.10 \\
\hline 4.1 & 0.08 & 0.09 \\
\hline 5.5 & 0.09 & 0.10 \\
\hline 7.1 & 0.09 & 0.10 \\
\hline SJ [30] & \multicolumn{2}{c}{0.09} \\
\hline
\end{tabular}

Beyond the potential core region, the spread rates of both the outer and inner regions are similar, and independent of spacing ratio. The results for the twin jets are also in very good agreement with the single jet value. Unlike the outer shear layer, however, the half-velocity width in the inner layer gradually deviates from the linear region, and the location where the deviation commences moved upstream with decreasing nozzle spacing, approximately about $5 d$ after $x_{m p}$. This observed deviation is consistent with the trend reported for round twin jets by [22], and shows that the jets' expansion in the inner region is enhanced due to the stronger interaction of the jets at a lower spacing ratios.

The vorticity thickness has been used to assess the growth of plane mixing layers [42] as well as separated and reattached turbulent shear flows such as backward facing step [43], forward facing step [44] and 3D surface-mounted bluff bodies [45]. Since the inner shear layers of twin jets bear some resemblance to these shear flows, their growth is also assessed using the vorticity thickness. The vorticity thickness $\delta_{w}$ is defined as follows:

$$
\delta_{w}=\frac{\Delta U}{\left(\frac{\partial U}{\partial y}\right)_{\max }}
$$

where $\Delta U=U_{c l}-U_{s y m}$ is the local maximum velocity difference and $\left(\frac{\partial U}{\partial y}\right)_{\max }$ is the local maximum velocity gradient evaluated in the inner shear layer. The influence of nozzle spacing 
on the distribution of $\delta_{w} / d$ is examined in Fig. 4.6. Similar to other shear layers, $\delta_{w} / d$ increases linearly with streamwise distance initially followed by a near-constant $\delta_{w} / d$ region. The streamwise location corresponding to the onset of the constant region increases with spacing ratio, and it is interesting to note that it is quite close to the merging point of the respective spacing ratio.

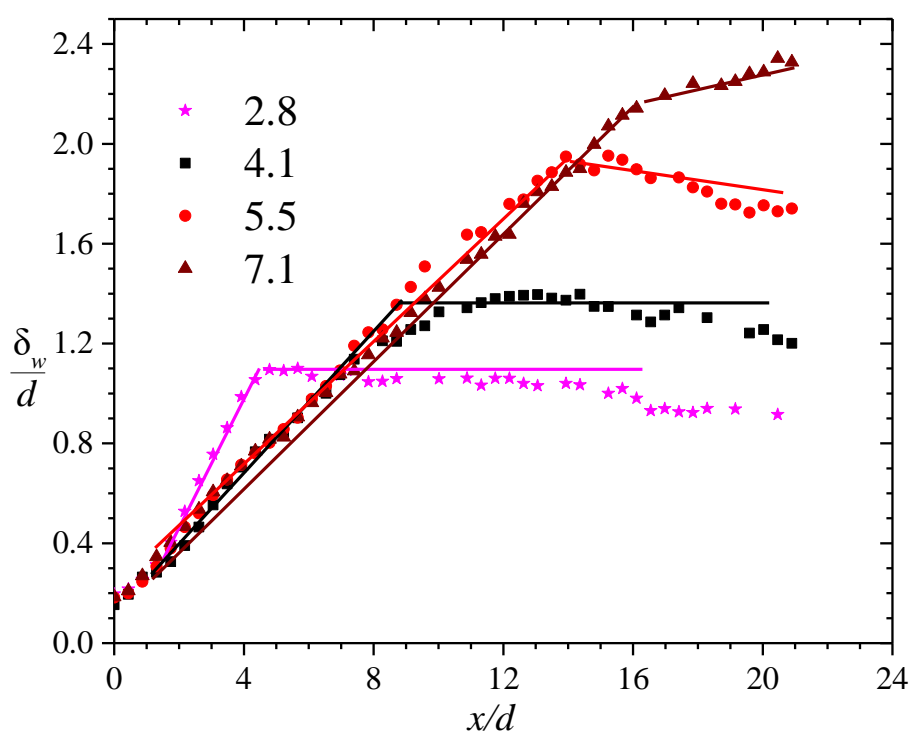

Figure 4.6: Local vorticity thickness along the jet's inner shear layer

Figure 4.6 clearly shows that the growth rate, $d \delta_{w} / d x$, diminishes as nozzle spacing is increased. The present growth rates for $S / d=2.8$ and 4.1 compare reasonably well with typical values of $0.14-0.22$ reported for plane mixing layers and separated and reattached shear flows.

Table 4.5 Vorticity thickness growth linear growth rate along the jet's inner shear layer

\begin{tabular}{cc}
\hline $\boldsymbol{S} / \boldsymbol{d}$ & $\boldsymbol{d} \boldsymbol{\delta}_{w} / \boldsymbol{d} \boldsymbol{x}$ \\
\hline 2.8 & 0.20 \\
\hline 4.1 & 0.14 \\
\hline 5.5 & 0.13 \\
\hline 7.1 & 0.11 \\
\hline SJ & 0.11 \\
\hline
\end{tabular}




\subsection{Reynolds Stresses, Turbulent Intensities and Turbulent Kinetic Energy}

Contour plots of Reynolds shear stress as well streamwise and transverse Reynolds normal stresses are shown in Fig. 4.7. Due to the remarkable similarity observed between the lower and upper jets (Figure 4.1), only the lower half of the flow domain is shown here.

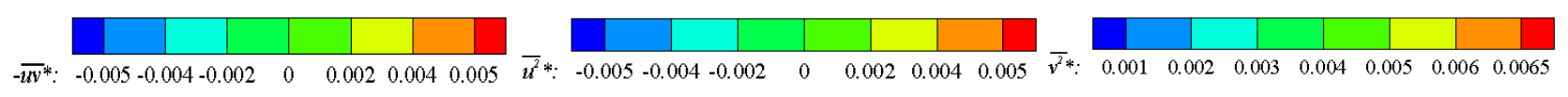

(a)

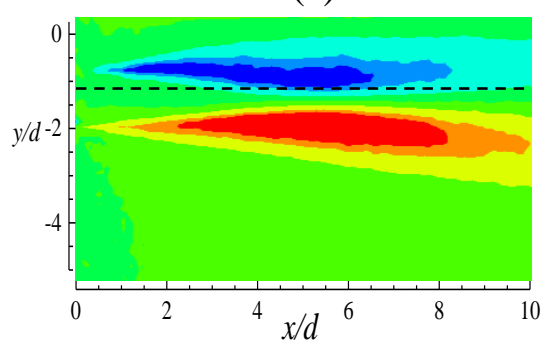

(d)

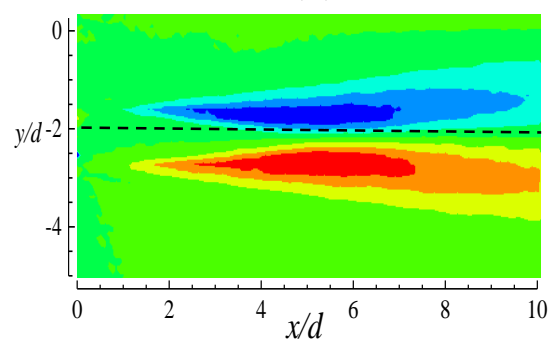

$(\mathrm{g})$

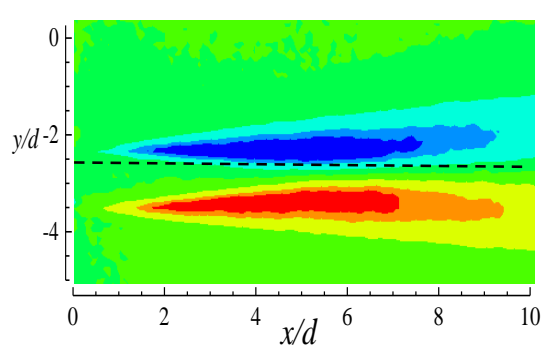

(j)

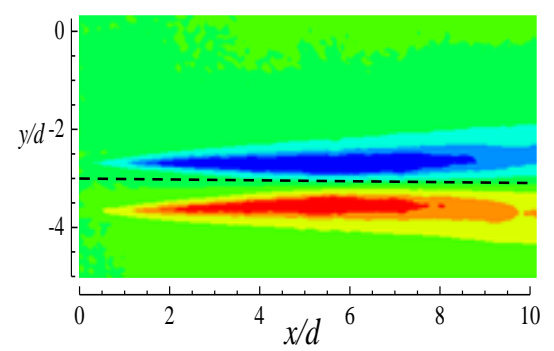

(b)

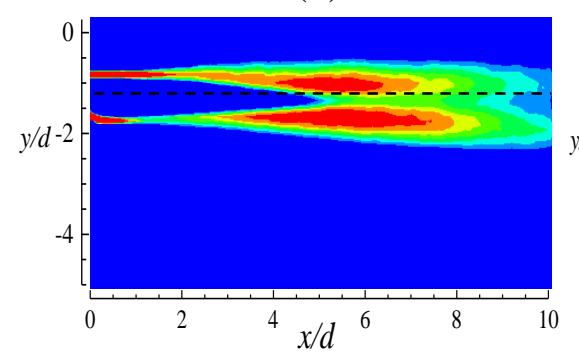

(e)

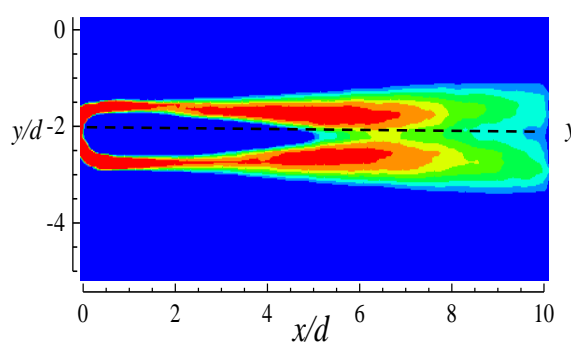

(h)

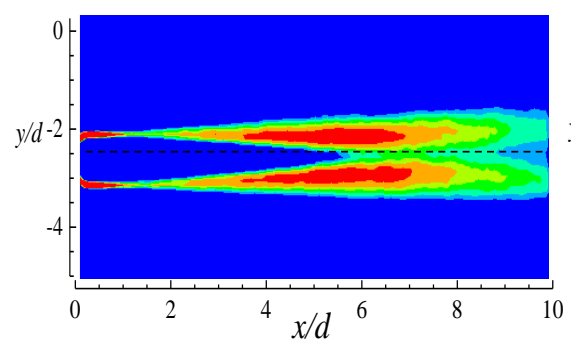

$(\mathrm{k})$

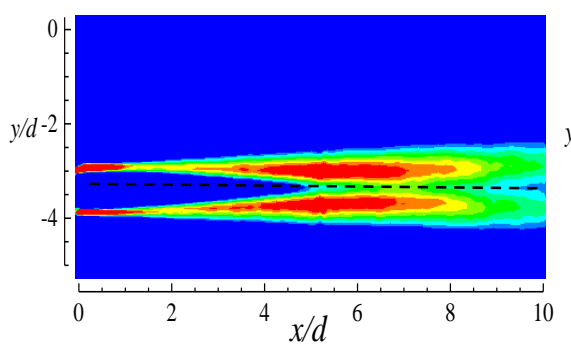

(c)

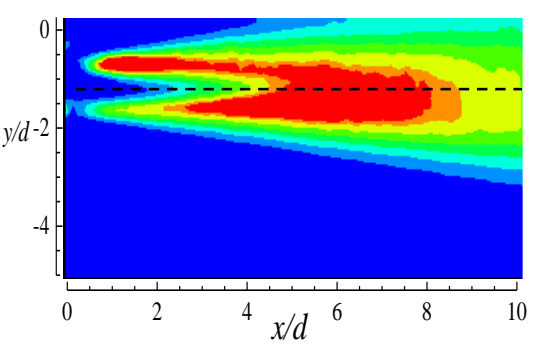

(f)

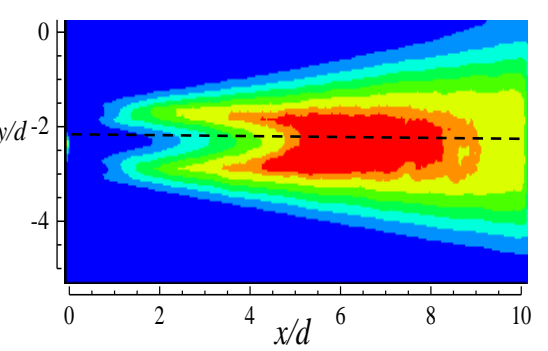

(i)

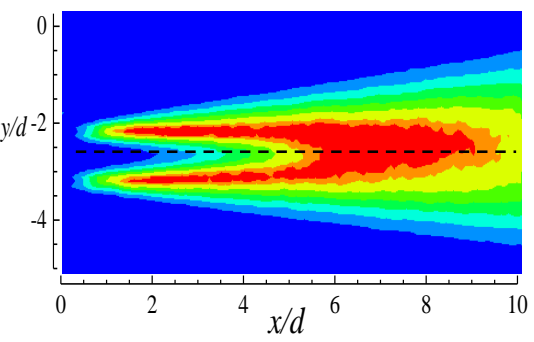

(1)

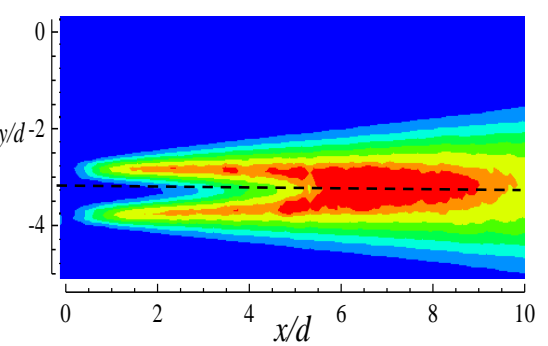

Figure 4.7: Contours of Reynolds shear stress, streamwise Reynolds normal stress and transverse Reynolds normal stress for $S / d=(\mathrm{a}, \mathrm{b}, \mathrm{c}) 2.8$, (d, e, f) 4.1, (g, h, i) 5.5 and (j, $\mathrm{k}, 1) 7.1$ 
It is noted that the Reynolds shear stress rises across the shear layers where the mean velocity shear is maximum and vanishes along the jet centerline where the mean velocity shear is zero. Also the opposite signs of the Reynolds shear stress in the inner and outer shear layers are because the Reynolds shear stress is negatively correlated to the mean velocity gradient. The Reynolds normal stress contours, on the other hand, exhibit a rise in magnitude that commences along the shear layers and gradually propagates towards the jet centerline. The level of the Reynolds shear and normal stresses in the outer shear layer is remarkably similar at all spacing ratios. This observation further shows that even though the proximity of the two jets may alter the extent of interaction between their inner shear layers, there exists no significant communication between the inner and outer layers, irrespective of nozzle spacing. At $S / d \geq 4.1$, the magnitudes of the Reynolds stresses in the inner shear layer are also not significantly different from the level observed in the outer shear layer. This is similar to a free single jet, and suggests that at larger spacing ratios, neighboring jets have negligible effect on each other across the shear layers. At $S / d=2.8$, however, the Reynolds normal stresses, particularly $\overline{v^{2}}$, in the inner layer upstream of $x_{m p}(x / d \leq 4)$ are relatively higher than observed in the outer shear layer. Downstream of $x_{m p}$, the opposite trend is observed where the normal and shear stresses in the inner layer are suppressed relative to outer shear layer levels. The relative suppression of normal stresses in the inner shear layers was also observed in twin plane jets [19].

The turbulent kinetic energy $(k)$ and its budget terms were examined to provide further insight into the observed enhancement in the Reynolds normal stress in the inner shear layer at $S / d=2.8$. Since a two-component planar PIV was used in the present experiments, the spanwise mean and fluctuating velocities were not available. Therefore, the production term $\left(P_{k}\right)$, convection by the mean flow $\left(-C_{k}\right)$ and turbulent diffusion term $\left(D_{k}\right)$ were approximated as follows: 


$$
\begin{gathered}
P_{k}=-\overline{u^{2}} \frac{\partial U}{\partial x}-\overline{v^{2}} \frac{\partial V}{\partial y}-\overline{u v}\left(\frac{\partial V}{\partial x}+\frac{\partial U}{\partial y}\right) \\
-C_{k}=-U \frac{\partial k}{\partial x}-V \frac{\partial k}{\partial y} \\
D_{k}=-\frac{\partial \overline{k u}}{\partial x}-\frac{\partial \overline{k v}}{\partial y}
\end{gathered}
$$

where $k$ is the turbulent kinetic energy and is approximated as $k \approx \frac{1}{2}\left(\overline{u^{2}}+2 \overline{v^{2}}\right)$. The dissipation rate was not evaluated because the spatial resolution is not adequate to sufficiently resolve the Kolmogorov scales, which are largely responsible for dissipation. Contour plots of $k^{*}, P_{k}^{*},-C_{k}^{*}$ and $D_{k}^{*}$ for $S / d=2.8$ and 7.1 are shown in Fig. 4.8. Figures $4.8 \mathrm{a}$ and $4.8 \mathrm{~b}$ show that the turbulent kinetic energy displays distinct offset peak values in the vicinity of the nozzles, but as the jets develop further downstream and the shear layers spread, the kinetic energy is transported towards the centerline and the double peaks tend to disappear. For $S / d=2.8$, there is a noticeable enhancement of turbulent kinetic energy in the inner shear layer upstream of $x_{m p}$ relative to the outer shear layer levels followed by a suppression, which are not apparent in the case of $S / d=7.1$. This should be expected in view of the trends observed in the Reynolds normal stresses in Fig. 4.7. It is clear from Fig. 4.8c and 4.8d that in the region $x / d \leq 4$, turbulence production levels in the inner and outer shear layers for the smallest spacing ratio, $S / d=2.8$, are comparable. Turbulent diffusion (Fig. 4.8e and 4.8f), although positive in the inner shear layers, is an order of magnitude lower than the production levels so that its contribution to the overall budget is negligible. 


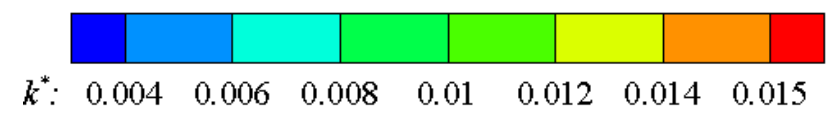

(a)

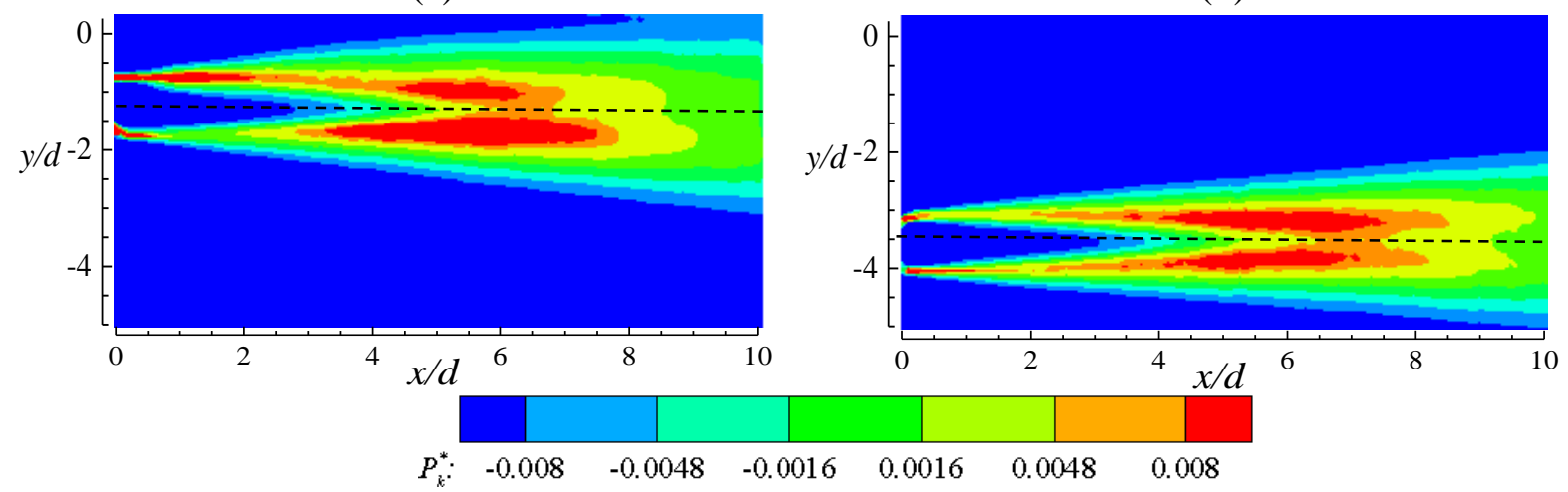

(c)

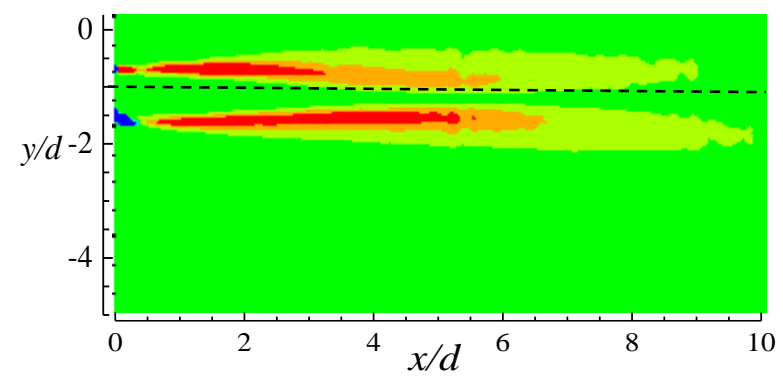

(d)

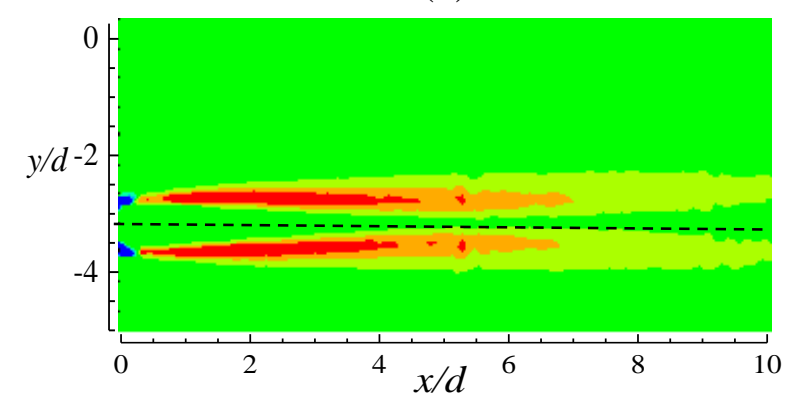

$\begin{array}{lllllll}D_{k}^{*}: & -0.0004 & -0.00024 & -8 \mathrm{E}-05 & 8 \mathrm{E}-05 & 0.00024 & 0.0004\end{array}$

(e)

(f)
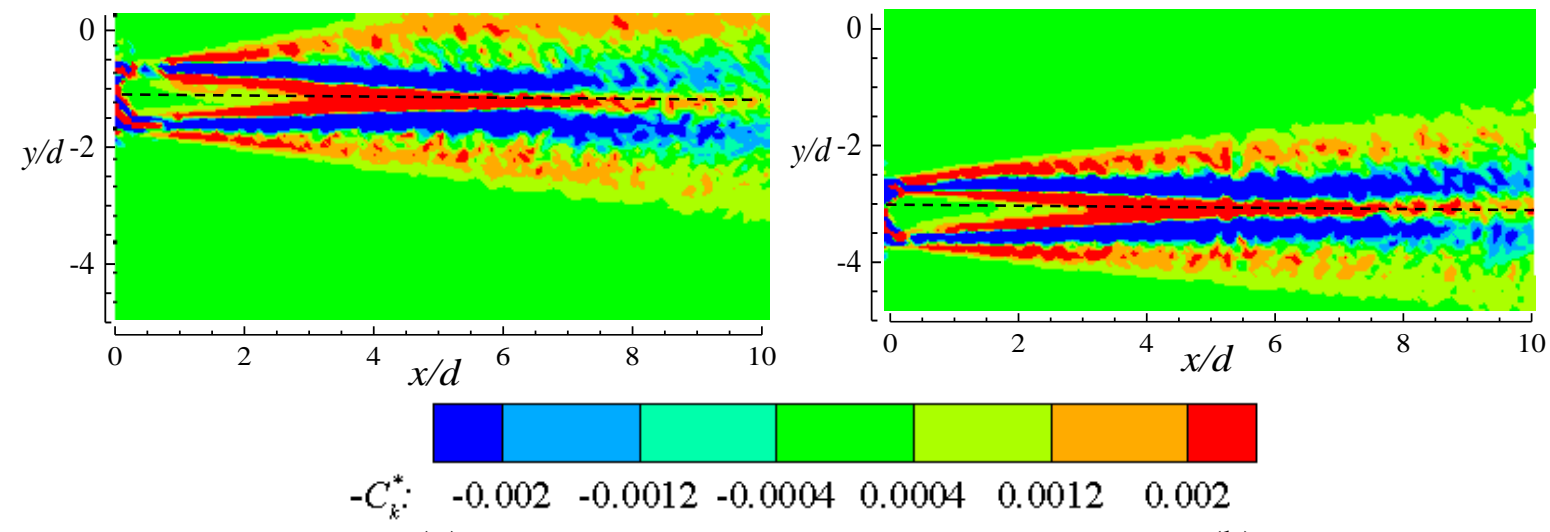

(g)

(h)
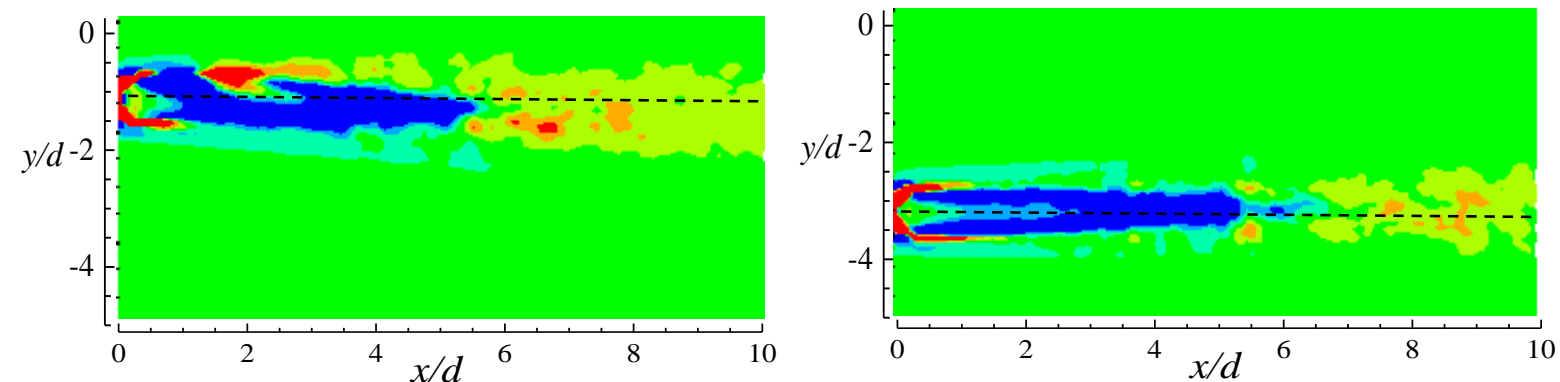

Figure 4.8: Contours of turbulent kinetic energy, production, convection and turbulent diffusion for $S / d=2.8\left(\mathrm{a}, \mathrm{c}, \mathrm{e}, \mathrm{g}\right.$ ) and $7.1(\mathrm{~b}, \mathrm{~d}, \mathrm{f}, \mathrm{h})$ normalized by $U_{\max } / d$ 
The convection term (Fig. 4.8g and 4.8h), however, is of the same order of magnitude as the production term, and contributes negatively to the overall $k$ budget except for $S / d=2.8$, where a pocket of positive contribution that is of comparable magnitude to the production term is observed. This positive contribution from the convection will provide additional source of energy to reinforce the production term. Thus, the relatively higher $k$ levels and subsequently high Reynolds normal stress levels observed in the inner shear layers for $S / d=2.8$ in the converging region are likely explained by the convective term.

Distributions of streamwise and transverse turbulent intensities along the jet centerline $\left(u_{r m s . c l}, v_{r m s, c l}\right)$ and symmetry plane $\left(u_{r m s . s y m}, v_{r m s, s y m}\right)$, normalized by $U_{\max }$ are shown in Fig. 4.9. For all spacing ratios, the turbulent intensities normalized by $U_{\max }$ increased rapidly and reached peak values of $u_{r m s, c l} / U_{\max }=0.12$ and $v_{r m s, c l} / U_{\max }=0.09$ at $x / d=7.5$. The location and magnitude of the maximum intensity values are also in very good agreement with the single jet case [9]. Subsequent to the rapid rise, the turbulent intensities monotonically decay and tend towards the symmetry plane values.
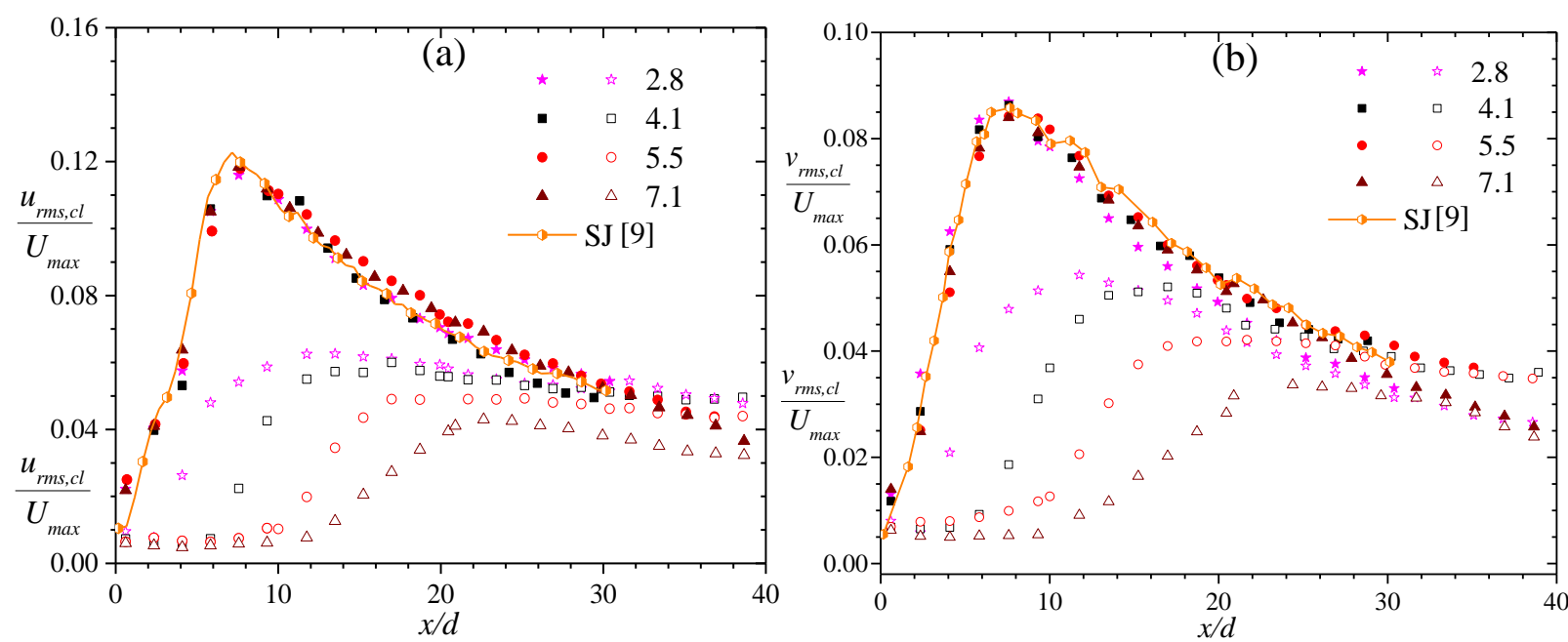

Figure 4.9: Development of (a) streamwise turbulent intensity, $u_{r m s} / U_{\max }$ and (b) transverse turbulent intensity, $v_{\text {rms }} / U_{\max }$ along symmetry plane and jet centerline 
It is interesting to note that the peak values of $u_{r m s, c l} / U_{\max }$ and $v_{r m s, c l} / U_{\max }$ for the present study are generally lower than $u_{r m s, c l} / U_{\max }=0.15-0.17$ and $v_{r m s, c l} / U_{\max }=0.12$ 0.14 reported for single round jets issued from OP nozzles [10,46], but compare quite favorably with those issued from SC nozzles [10,25]. The turbulent intensities along the symmetry plane, on the other hand, are initially zero, followed by a rise to a maximum value near the merging point and a subsequent decay towards the centerline values at the combined point, in a similar fashion to $U_{\text {sym }} / U_{\max }$ (Fig. 4.2a). Unlike the centerline turbulent intensities, significant effects of nozzle spacing are clearly evident along the symmetry plane. For example, the peak values of the streamwise turbulent intensity for $S / d=2.8,4.1,5.5$ and 7.1 are $0.062,0.057$, 0.049 and 0.043 (Fig. 4.9a), respectively, and the corresponding peak values of the transverse turbulent intensities are $0.054,0.052,0.043$ and 0.033 (Fig. 4.9b), respectively. The location of the peak values for both streamwsie and transverse intensities are $x / d=12,16,18$ and 24 for $S / d=2.8,4.1,5.5$ and 7.1, respectively. These results indicate a more rapid rise and higher peak value of turbulent intensities at lower spacing ratios which is similar to the trend reported by [25]. Also, the turbulent intensities along the symmetry plane attained their peak values sooner than the streamwise mean velocity did which is consistent with the claim made by [25] that the mutual interaction between the inner layers appears faster in the turbulent intensities than in the mean velocity.

The turbulent intensities along the jet centerline $\left(u_{r m s . c l}^{*}, v_{r m s, c l}^{*}\right)$, normalized by $U_{c l}$, are shown in Fig. 4.10. Both the streamwise and transverse turbulent intensities increase monotonically along the centerline to an asymptotic value. The rate of increase of the streamwise intensities is independent of nozzle spacing, while for the transverse intensities, the rate of increase for $S / d=2.8$ is approximately $31 \%$ higher than that of $S / d=4.1,5.5$ and 7.1. 

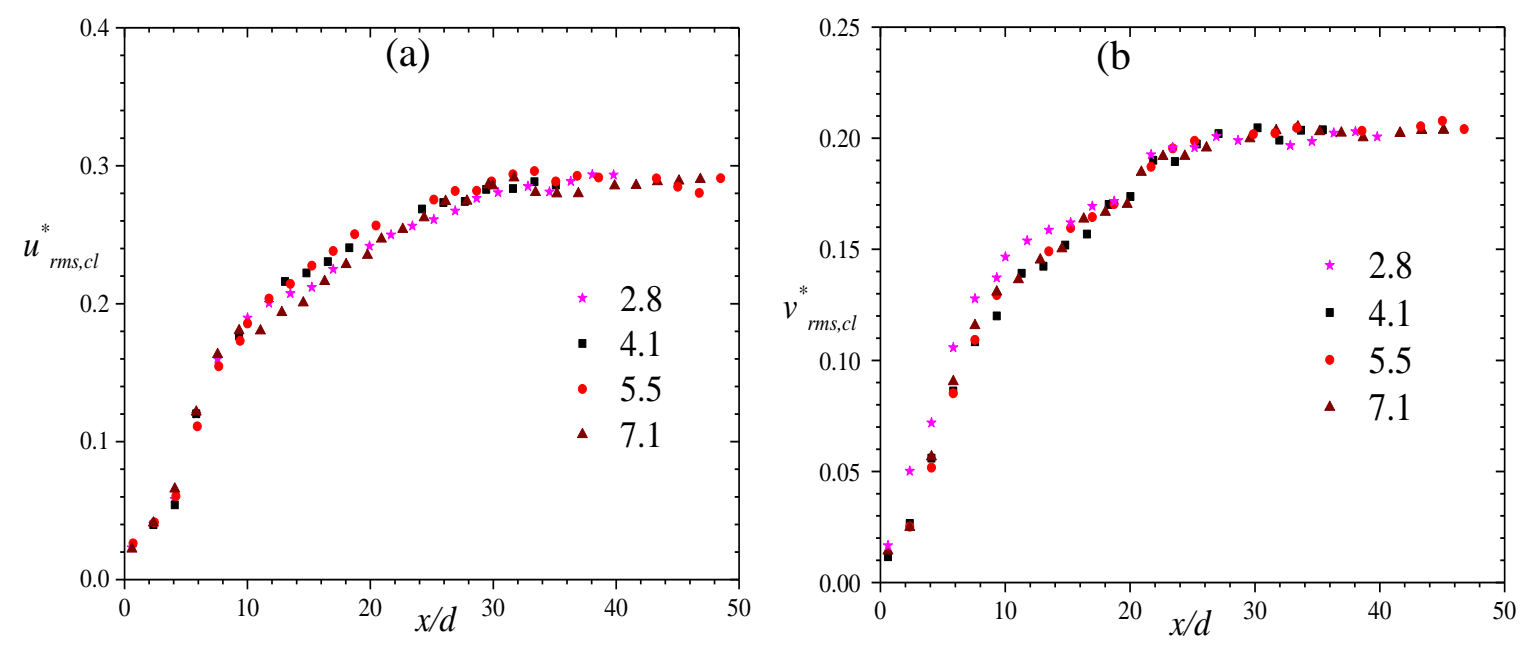

Figure 4.10: (a) Development of $u_{r m s}$ and (b) $v_{r m s}$ normalized by $U_{c l}$ along the jet centerline

In both cases, the turbulent intensities increase to self-similar values of $u_{r m s, c l}^{*}=0.28$ and $v_{r m s, c l}^{*}=0.20$. These values are higher than $u_{r m s, c l}^{*}=0.21-0.26$ and $v_{r m s, c l}^{*}=0.15$ reported for single round jets issued from OP nozzles $[11,15,47,48]$ but compare favorably with $u_{r m s, c l}^{*}=$ $0.25-0.28$ and $v_{r m s, c l}^{*}=0.18-0.20$ for jets issued from SC nozzles $[14,49,50]$. Moreover, the present results do not show a localized hump upstream of the self-similar state. The absence of the hump reflects the absence of two phenomena. The first phenomenon is axis-switching which is otherwise apparent for geometries such as rectangular, elliptic and triangular nozzles [15]. The second phenomenon is the collision of large-scale vortical structures along the inner shear layers downstream of the potential core region, which would produce large-scale entrainment of ambient fluid and result in higher velocity fluctuations [50,51].

The turbulent intensities along the symmetry plane ( $\left.u_{r m s . s y m}^{*}, v_{r m s, s y m}^{*}\right)$ (Fig. 4.11) collapsed onto universal curves and again demonstrate the suitability of the scaling adopted herein. The profiles in these figures follow Eqn. (4.1) in the merging region with the fitting coefficients summarized in

Table 4.6. 

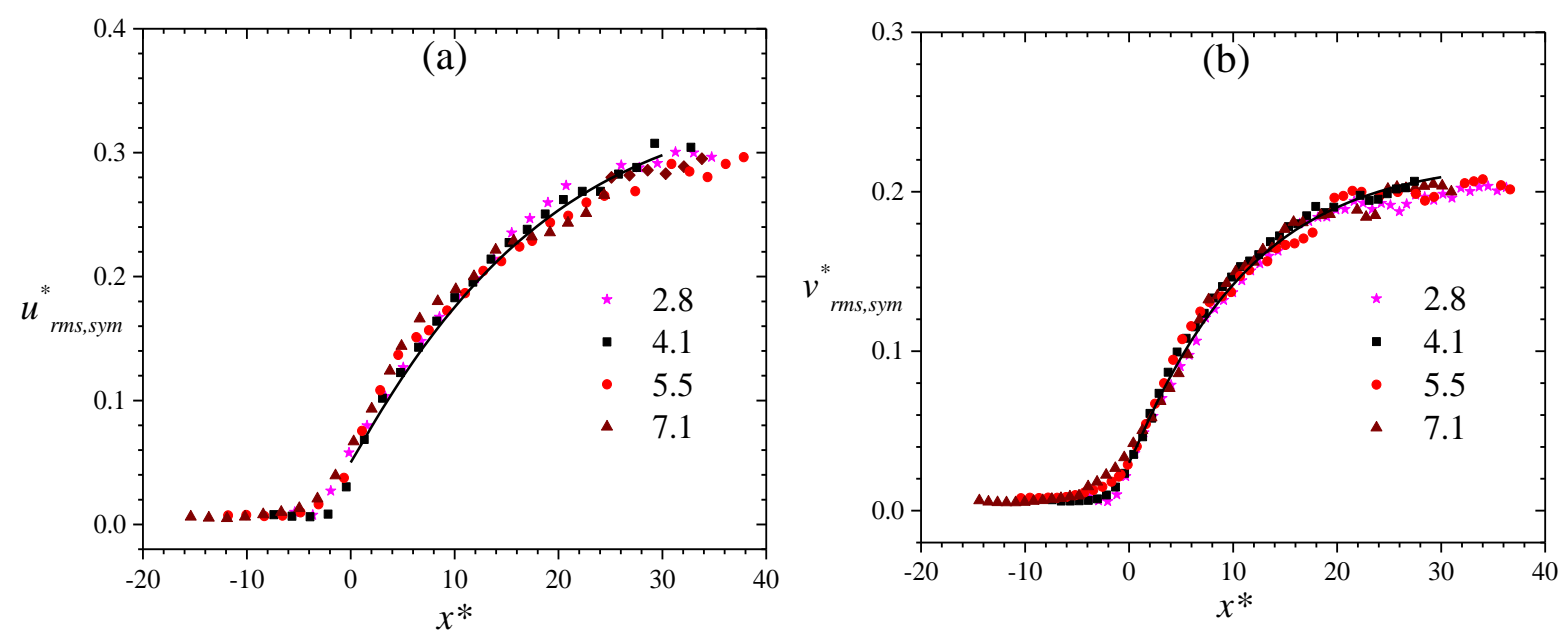

Figure 4.11: Scaling of (a) $u_{r m s}$ and (b) $v_{r m s}$ along the symmetry plane

Table 4.6 Summary of fitting coefficients for streamwise and transverse turbulent intensities' scaling along the symmetry plane according to Eqn. (4.1)

\begin{tabular}{lccccc}
\hline & $\mathbf{A}$ & $\mathbf{B}$ & $\mathbf{C}$ & $\mathbf{D}$ & $\mathbf{E}$ \\
\hline $\boldsymbol{u}_{\boldsymbol{r m s}, \boldsymbol{s y m}}^{*}$ & 0.77 & 0.23 & 1 & 15 & 0.95 \\
& & & & & \\
\hline $\boldsymbol{v}_{\boldsymbol{r m s , s y m}}^{*}$ & 0.84 & 0.16 & 1 & 10 & 0.97 \\
\hline
\end{tabular}

The ratio of Reynolds normal stresses $\left(\overline{u^{2}} / \overline{v^{2}}\right)$ is routinely used to assess the large-scale anisotropy of turbulent shear flows. For isotropic turbulence, $\overline{u^{2}} / \overline{v^{2}}=1$. The stress ratio along the jet centerline is presented in Fig. 4.12. In the vicinity of the nozzle, it is observed that the anisotropy is at its highest level for all spacing ratios due to the low $\overline{v^{2}}$ levels in that region relative to $\overline{u^{2}}$. In the potential core region, $(2 \leq x / d \leq 4), \overline{v_{c l}^{2}}$ levels rise at approximately $29 \%$ faster rate than $\overline{u_{c l}^{2}}$ for $S / d=2.8$ (Figs. 4.10a and 4.10b), and as a consequence, $\overline{u_{c l}^{2}} / \overline{v_{c l}^{2}}$ decreases from 3 to a minimum value of 0.9 at $x / d \approx 4$. At higher spacing ratios, the difference in rates of increase between $\overline{v_{c l}^{2}}$ and $\overline{u_{c l}^{2}}$ reduces leading to minimum values of 1,2 and 4 for $S / d=4.1,5.5$ and 7.1. Downstream of potential core region, the stress ratio reaches a nearconstant values of $1.8 \pm 0.2$ for all spacing ratios. This value is comparable to a value of 1.3 reported by free single jet studies [46,52] and 1.4 reported for a plane twin jets [18]. 


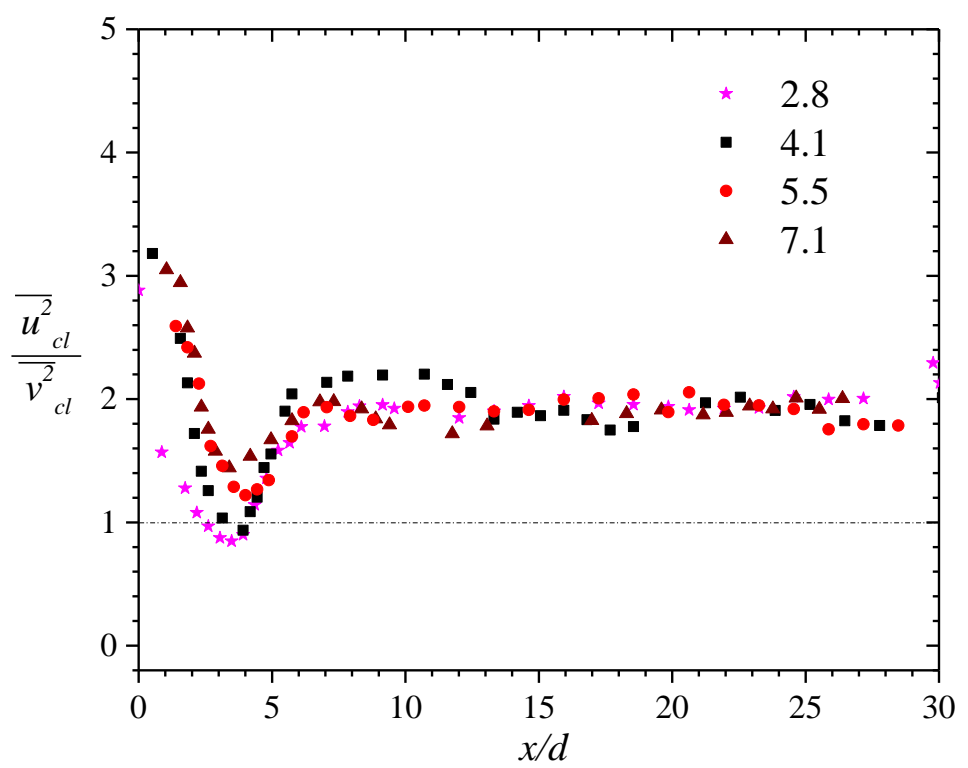

Figure 4.12: Reynolds normal stresses ratio along jet centerline

\subsection{Skewness and Flatness Factors}

The skewness, $S u=\overline{u^{3}} / u_{r m s}^{3}$ and flatness factor, $F u=\overline{u^{4}} / u_{r m s}^{4}$ provide a measure of the asymmetry and flatness, respectively, of the probability density function. In turbulent shear flows, non-Gaussian skewness $(S u \neq 0)$ indicates that larger velocity fluctuations in one direction are more probable than in the other, while non-Gaussian flatness $(F u \neq 3)$ generally aids in identifying regions of high turbulent intermittency, for example, occasional intrusions of low-velocity ambient fluid into the jet due to the growth of more coherent and large scale roller-like structures through the shear layers $[15,53]$. The skewness and flatness factors of the streamwise and transverse fluctuations along the jet centerline are shown in Fig. 4.13. The skewness and flatness along the centerline of a round single jet performed under the same test conditions is also included for comparison. 

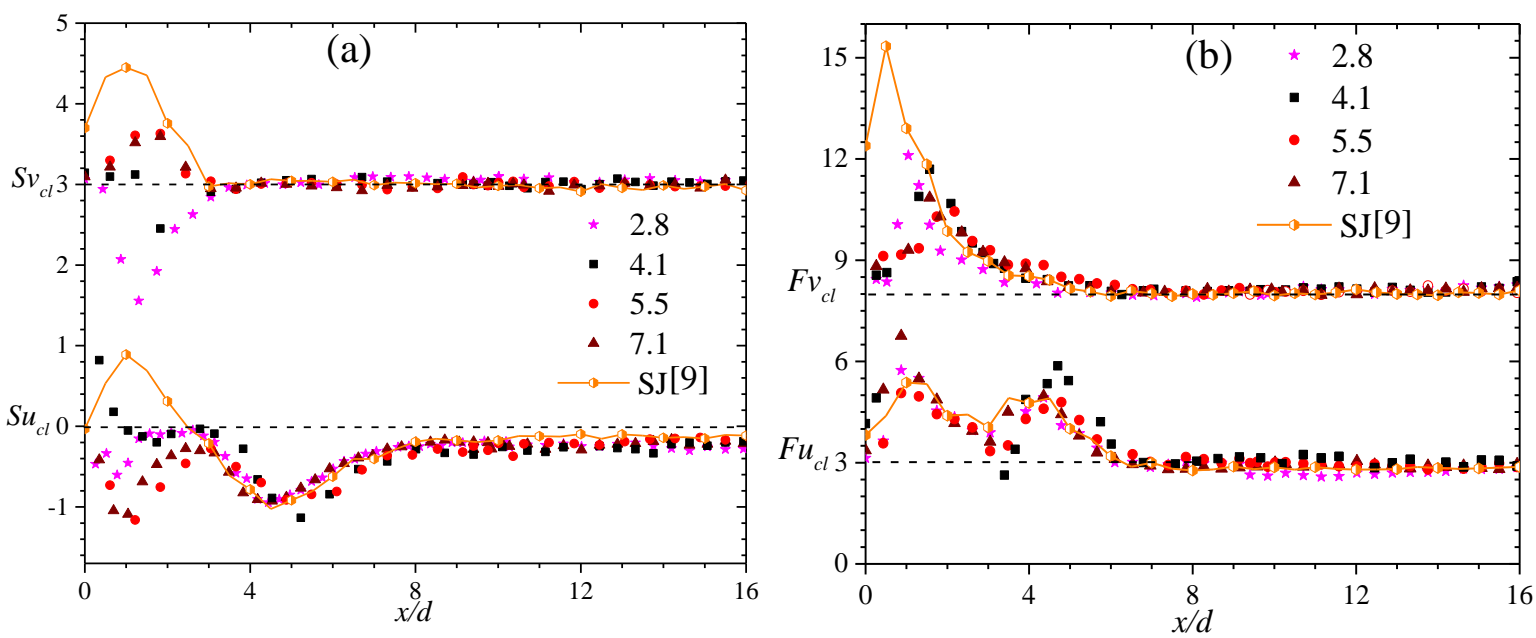

Figure 4.13: Streamwise and transverse mean velocity (a) skewness ( $S u$ and $S v)$ and (b) flatness $(F u$ and $F v)$ along the jet centerline

Along the centerline, Su drifts away from the Gaussian value within the potential core region $(0 \leq x / d \leq 4)$, exhibiting negative values. Negative skewness in this region suggests that either low speed or momentum fluctuations are more probable than high momentum fluctuations or the presence of rare but large low momentum spikes. This behavior, together with the highly non-Gaussian flatness values is a clear evidence of high turbulent intermittency in the potential core region. Beyond the potential core, $S u$ and $F u$ tend to asymptotic values that are comparable to Gaussian distributions, and suggests a breakdown of the large scale turbulence motions due to entrainment of ambient fluid into the jet core. No noticeable effects of nozzle spacing ratio are found in the distributions of $S u$ and $F u$ along the centerline, and the present results are also in good agreement with the single jet, expect in the vicinity of the nozzle exit.

The skewness and flatness of the transverse velocity fluctuations along the jet centerline also reveal highly non-Gaussian distribution in the potential core region followed by Gaussian distributions at $x / d \geq 4$. Unlike $S u$, however, the magnitude of $S v$ varies with spacing ratio in the potential core region. Particularly, in the region $x / d \leq 2, S v$ exhibits highly negative skewness for $S / d=2.8$, which suggests that outflow fluctuations have larger magnitudes than 
the inflows. The outflow fluctuations become milder for $S / d=4.1$ and eventually the inflow fluctuations grow larger than the outflows for $S / d=5.5$ and 7.1 and the single jet case. The inflow/outflow fluctuation magnitude difference, however, does not significantly affect the intermittency of the transverse velocity fluctuations, as reflected in $F v$ profiles. It is also observed that $F v$ for the twin jets is comparatively lower than its single jet counterpart, suggesting that the formation of coherent structures through the shear layers is suppressed by the proximity of the neighboring jet.

Figure 4.14 shows the skewness and flatness factors of the streamwise and transverse fluctuations along the symmetry plane, where the abscissa $x^{*}$ is measured relative to $x_{m p}$. As shown, the skewness and flatness factors are sensitive to nozzle spacing in the vicinity of $x_{m p}$. In this region, $S / d=2.8$ exhibits approximately $50 \%, 70 \%$ and $100 \%$ higher skewness $\left(S u_{s y m}\right)$ than its counterparts for $S / d=4.1,5.5$ and 7.1 , respectively. The highest flatness $\left(F u_{s y m}\right)$ is also observed for $S / d=2.8$. A similar trend is observed for $S v_{s y m}$ and $F v_{s y m}$. Beyond the merging point, all the profiles tend to Gaussian values reflecting the breakdown of the large-scale coherent structures upon the mixing of the jets' shear layers.
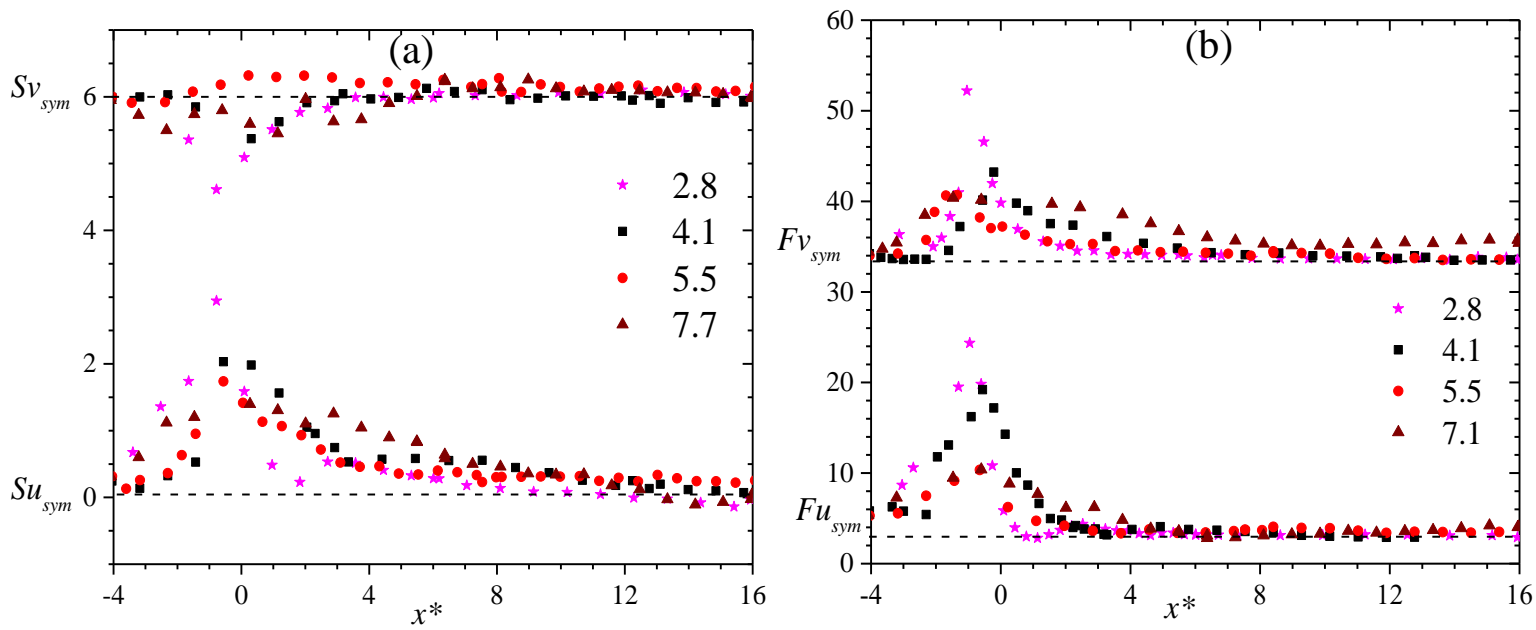

Figure 4.14: Streamwise and transverse mean velocity (a) skewness ( $S u$ and $S v)$ and (b) flatness $(F u$ and $F v)$ along the symmetry plane 


\subsection{Mean Velocities and Reynolds Stresses across the Shear Layers}

One-dimensional profiles of the streamwise mean velocity $U^{*}$, Reynolds normal stresses $\overline{u^{2 *}}$ and $\overline{v^{2 *}}$, and Reynolds shear stress $\overline{u v^{*}}$ across the shear layers within the converging (open symbols), merging (solid symbols) and combined (shaded symbols) regions are plotted in Fig. 4.15. The single jet profiles [9] at $x / d=25$, where the mean velocity has reached a self-similar state, are also shown for comparison. The ordinate $y$ ' is measured relative to the jet centerline and is normalized by $y_{+0.5}$, while the abscissa is normalized by $U_{c l}$.
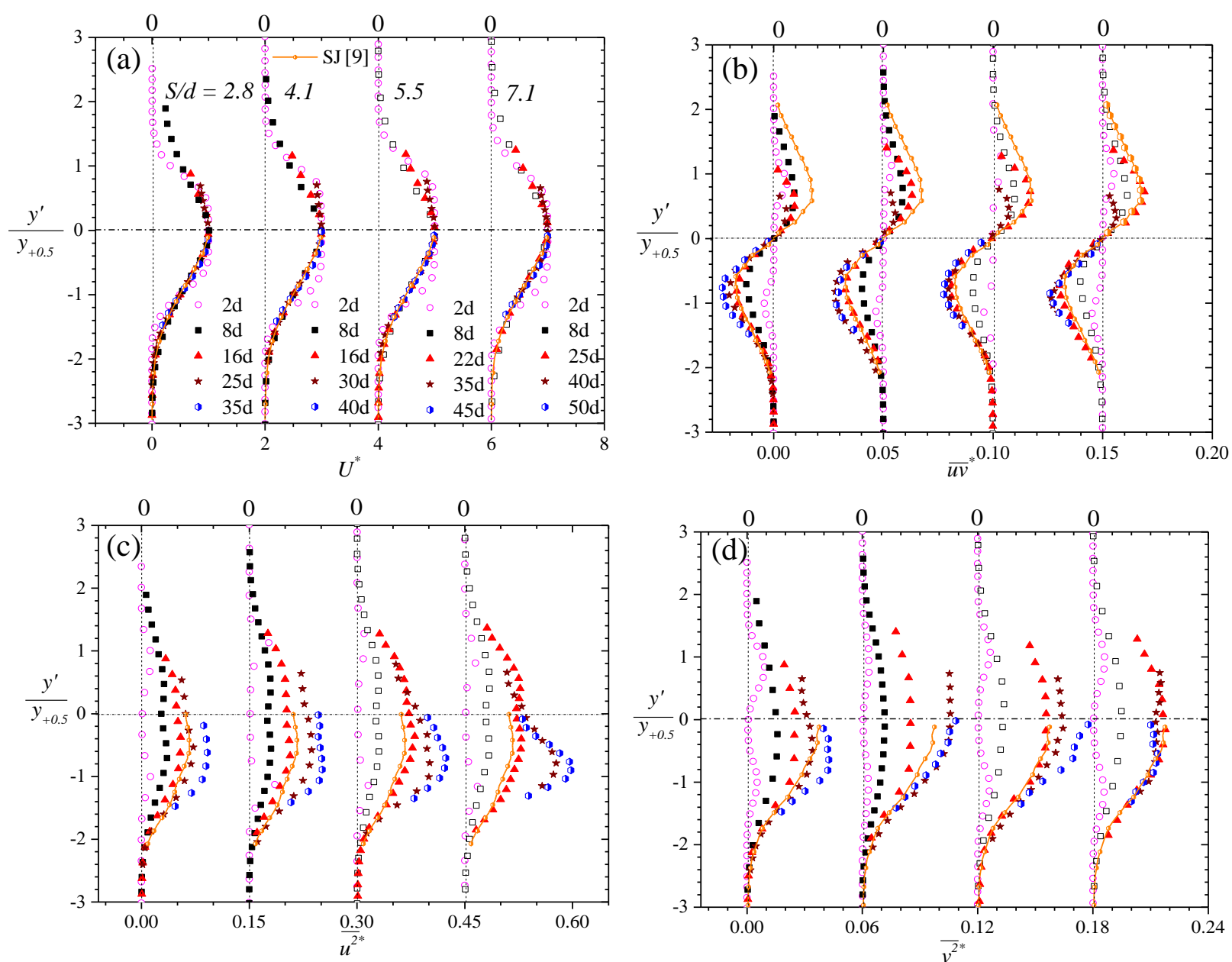

Figure 4.15: One-dimensional profiles of dimensionless (a) streamwise velocity (b) Reynolds shear stresses (c) streamwise normal stresses and (d) transverse normal stresses across select streamwise locations

At $x / d=2$, the mean velocity profiles (Fig. 4.15a) show a top-hat profile, similar to previous SC nozzle studies, and extend only a narrow region, irrespective of spacing ratio. At $x / d \geq 8$, 
the outer shear layer expands at a similar rate for all spacing ratios. However, higher velocities are observed in the inner shear layer at lower spacing ratios due to a faster spreading in that region. This is consistent with the half-velocity widths observed in Figs. 4.5a and 4.5b. In the combined region, the distinct twin jet profiles merged into a combined profile and collapse very well with the self-similar single jet profile.

The Reynolds shear stress profiles (Fig. 4.15b) show distinct peaks around the half-velocity width locations where the momentum exchange between the jet and ambient fluid is at a maximum and zero values along the centerline. As the jets evolve, $\overline{u v^{*}}$ levels grow indicating a higher rate of momentum exchange. However, the Reynolds shear stress grows at a slower rate in the inner layer than in the outer shear layer, and the suppression is more prominent at lower spacing ratios. The relative suppression of shear stress in the inner shear layers was also observed by [25]. In spite of this observation, the distributions of the Reynolds shear stress are akin to single jets, where the transverse location of $\overline{u v^{*}}=0$ coincides with location of the maximum mean velocity (i.e., $d U / d y=0$ ). An important implication of this observation is that, the dissimilarity between the Reynolds shear stress in the inner and outer shear layers does not preclude simple eddy viscosity models, $-u v=v_{t}(\partial U / \partial y+\partial V / \partial x)$ where $v_{t}$ is the kinematic eddy viscosity, to predict the mean flow behavior. This is in contrast with wall jets [54-56] where the wall-normal locations of $\overline{u v^{*}}=0$ and $d U / d y=0$ do not coincide due to the varying strength between the more turbulent outer shear layer and the less turbulent inner shear layer of the wall jet, and as a consequence, a region of counter-gradient diffusion or negative eddy viscosity occurs.

The Reynolds normal stress profiles (Figs. $4.15 \mathrm{a}$ and $4.15 \mathrm{~b}$ ) at $x / d=2$ exhibit distinct peaks around the half-velocity width locations. As the jets evolve further downstream $(x / d \geq 8)$, the peaks become less distinct partly due to the growth of the stresses along the centerline. Note that the peak levels of $\overline{v^{2 *}}$ are almost half of the corresponding $\overline{u^{2 *}}$ levels, and tend to 
propagate towards the jet centerline at a faster rate than $\overline{u^{2 *}}$. At $x / d=2$, the peak levels of $\overline{v^{2 *}}$ in the inner shear layer are in reasonable agreement with those in the outer shear layer for $S / d$ $=4.1,5.5$ and 7.1 but are $73 \%$ higher than its corresponding outer layer values for $S / d=2.8$ due to the contribution of the convective term described in Section 4.2. In the merging region, the suppression of the streamwise Reynolds normal stresses in the inner shear layer relative to the outer layer commences at $x / d=8,16,22$ and 40 for $S / d=2.8,4.1,5.5$ and 7.1 , and persists until the outer and inner stress peaks combine at the jet centerline. In the combined region, it is observed that $\overline{u^{2 *}}$ maintains its peak levels away from the jet centerline, especially at higher spacing ratios, in qualitative agreement with plane twin jets [18]. This behavior is a result of high stress production levels in the combined jet's shear layers that persist well beyond the combined point.

\subsection{Two-point Correlation and Integral Length Scales}

Two-point correlations of the streamwise and transverse velocity fluctuations ( $u$ and $v$ ) are used to characterize the spatial coherence of the large-scale turbulence motions and the integral length scales. The two-point auto-correlation function $\left(R_{A B}\right)$ between any two arbitrary quantities $A(x, y)$ and $B(x, y)$ is evaluated as follows:

$$
R_{A B}\left(x_{r e f}+\Delta x, y_{r e f}+\Delta y\right)=\frac{A\left(x_{r e f}, y_{r e f}\right) B\left(x_{r e f}+\Delta x, y_{r e f}+\Delta y\right)}{\sigma_{A}\left(x_{r e f}, y_{r e f}\right) \sigma_{B}\left(x_{r e f}+\Delta x, y_{r e f}+\Delta y\right)}
$$

where $\left(x_{r e f}, y_{r e f}\right)$ is the reference location, $\Delta x$ and $\Delta y$ are the spatial separations between $A$ and $B$ in the streamwise and transverse directions, respectively, and $\sigma_{\mathrm{A}}$ and $\sigma_{B}$ are the root mean square values of $A$ and $B$ at $\left(x_{r e f}, y_{r e f}\right)$ and $\left(x_{r e f}+\Delta x, y_{r e f}+\Delta y\right)$, respectively. The two-point correlation functions were calculated for each PIV interrogation area and then ensembleaveraged point by point. Figure 4.16 shows contours of the two-point auto-correlation function for the streamwise and transverse velocity fluctuations $\left(R_{u u}\right.$ and $\left.R_{v v}\right)$ at the merging point. 


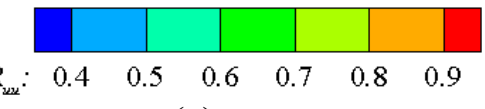

(a)

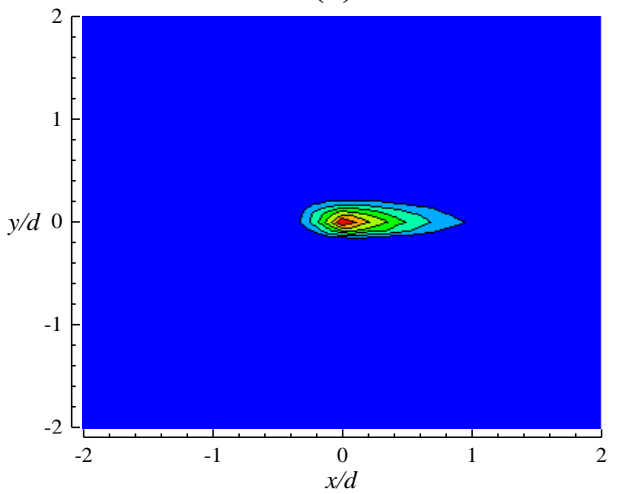

(c)

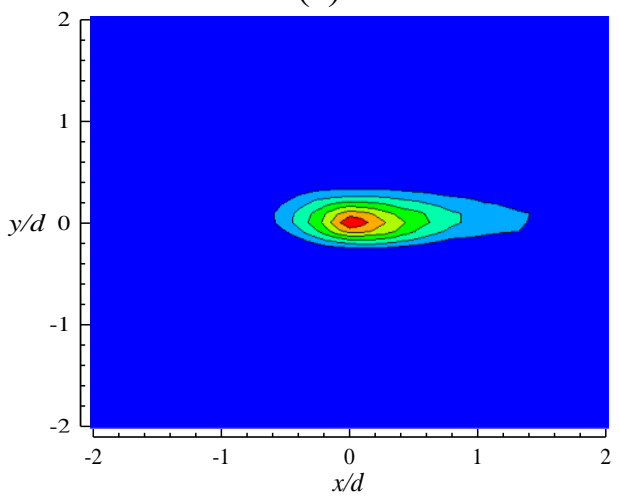

(e)

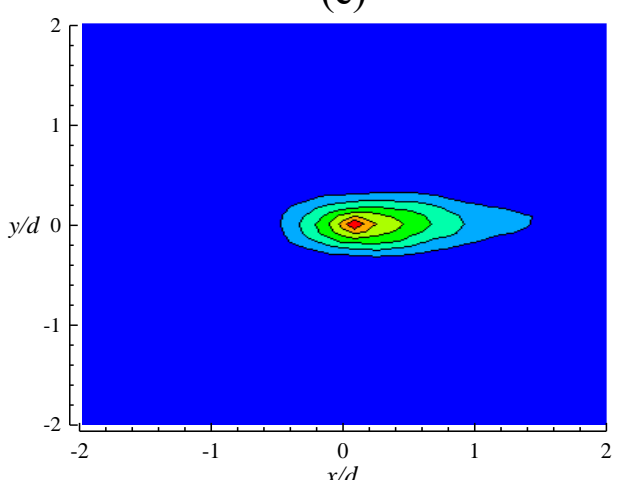

$(\mathrm{g})$

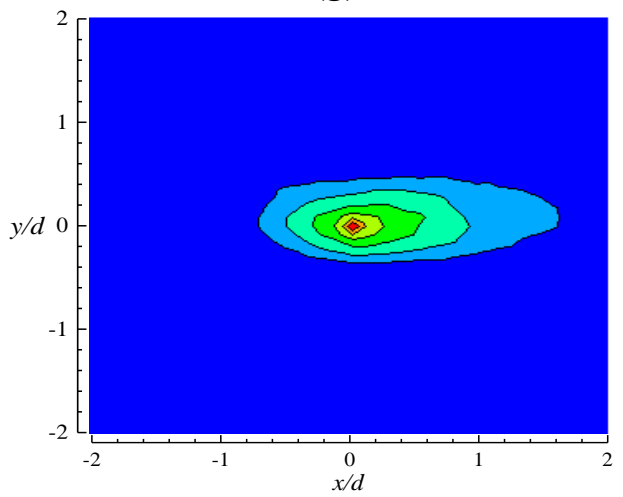

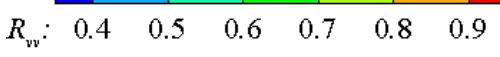

(b)

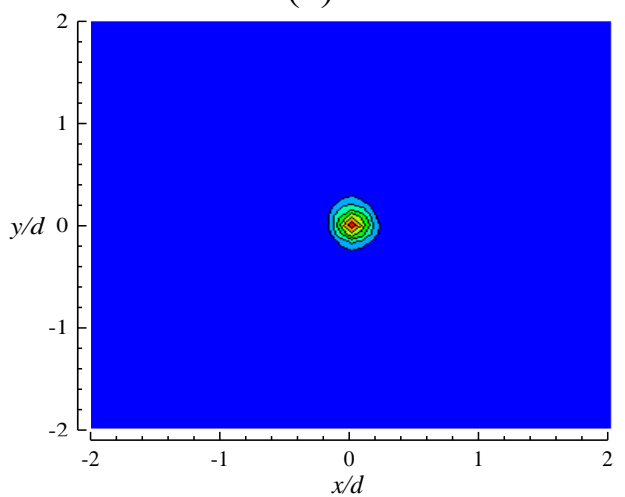

(d)

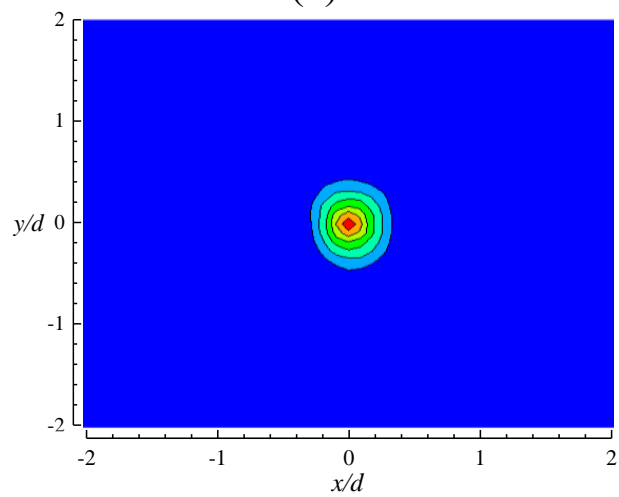

(f)

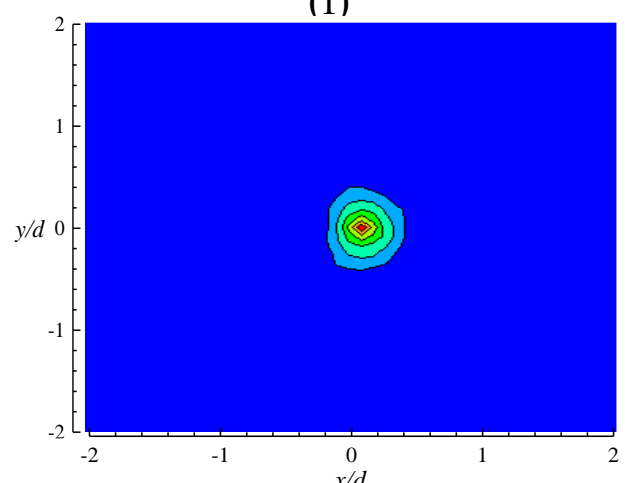

(h)

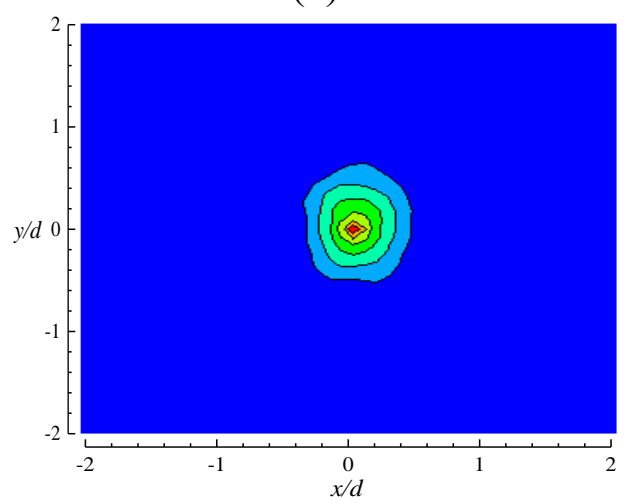

Figure 4.16: Contours of the two-point correlation of streamwise velocity fluctuation and transverse velocity fluctuation at the merging point for $S / d=$ $2.8(\mathrm{a}, \mathrm{b}), 4.1(\mathrm{c}, \mathrm{d}), 5.5(\mathrm{e}, \mathrm{f})$ and $7.1(\mathrm{~g}, \mathrm{~h})$ 
It is noted that the contours of $R_{u u}$ are elliptical, and elongated in the streamwise direction while $R_{v v}$ contours are more compact and rounded than those of $R_{u u}$. The contours also show that the physical size of turbulence structures embodied in $R_{u u}$ increase with spacing ratio, and also serves as an indication that the vortical structures become more coherent with increasing spacing ratio at the onset of shear layer mixing.

While the iso-contours provide a qualitative assessment of the size of large-scale structures, the size can be quantified by determining the streamwise $\left(L_{T x}\right)$ and transverse $\left(L_{T y}\right)$ integral length scales which are defined as follows [3]:

$$
\begin{aligned}
& L_{T x}=\int_{x_{r e f}}^{x_{0}^{\prime}} R_{u u}(x) d x \\
& L_{T y}=\int_{y_{r e f}}^{y_{0}^{\prime}} R_{u u}(y) d y
\end{aligned}
$$

where $x_{r e f}$ and $y_{r e f}$ coordinates of the self-correlation point, while $x_{0}$ and $y_{0}$ are the locations where the correlation takes a zero value downstream and above of the self-correlation point, respectively. Figure 4.17 shows one-dimensional plots of $R_{u u}(x)$ and $R_{v v}(y)$ at the merging point locations. As can be observed, there are instances where $R_{u u}(x)$ and $R_{v v}(y)$ do not reach zero correlation, therefore, following [57-59], Equations 12 and 13 can be modified to:

$$
\begin{gathered}
L_{T x}=x_{0.5}^{\prime}-x_{-0.5}^{\prime} \\
L_{T y}=y_{0.5-}^{\prime}-y_{-0.5}^{\prime}
\end{gathered}
$$

where $x^{\prime} 0.5$ and $x_{-0.5}^{\prime}$ are the locations where the correlation is 0.5 downstream and upstream of the self-correlation point, respectively, while $y_{0.5}^{\prime}$ and $y_{-0.5}^{\prime}$ are the locations where the correlation is 0.5 above and below the self-correlation point, respectively, indicated by the dashed lines. 


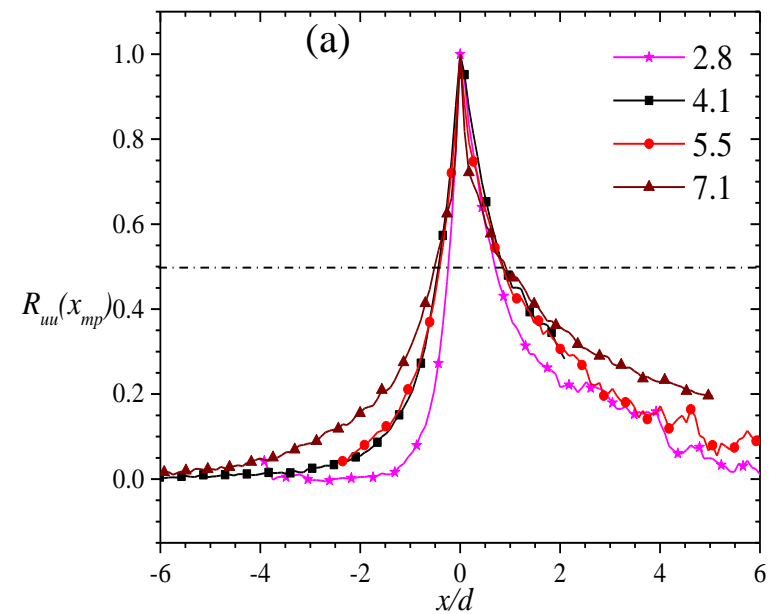

Figure 4.17: One-dimensional profiles of (a) $R_{u u}(x)$ and (b) $R_{v v}(y)$ at the merging point locations.

The results show that for all spacing ratios, $L_{T x}$ is approximately twice the size of $L_{T y}$, and increases with spacing ratio. Although $L_{T y}$, does not exhibit the same steady increase that $L_{T x}$ does, it is apparent that the transverse length scales also increase with increasing spacing ratio. Table 4.7 summarizes the $L_{T x}$ and $L_{T y}$ values obtained from Fig. 4.17 according to Eqns. (4.9) and (4.10).

Table 4.7 Streamwise and transverse integral length scales at merging point locations

\begin{tabular}{ccc}
\hline $\boldsymbol{S} / \boldsymbol{d}$ & $\boldsymbol{L}_{\boldsymbol{T x}}\left(\boldsymbol{x}_{\boldsymbol{m} \boldsymbol{p}}\right)$ & $\boldsymbol{L}_{\boldsymbol{T y}}\left(\boldsymbol{x}_{\boldsymbol{m} \boldsymbol{p}}\right)$ \\
\hline 2.8 & 0.95 & 0.41 \\
\hline 4.1 & 1.27 & 0.64 \\
\hline 5.5 & 1.29 & 0.58 \\
\hline 7.1 & 1.43 & 0.82 \\
\hline
\end{tabular}

The variation of streamwise and transverse integral length scales along the upper jet centerline is presented in Fig. 4.18. Results from the round single jet obtained at $\operatorname{Re}=10,000$ in the same facility [30] are also included for comparison. The integral length scales are relatively small near the jet exit and become larger with increasing streamwise distance. In the present study, the values of $L_{T x(c l)}$ increase linearly with an average slope of $0.092 \pm .005$ up to the combined point. This value is approximately two times higher than the single jet linear 
growth rate $(0.042)$, which indicates that the vortical structure along the twin jet centerline are more coherent in the streamwise direction than that of the single jet. Beyond the combined point, the growth rate drops to 0.025 for $S / d=7.1$, while for the lower spacing ratios, the structures actually shrink in size at a rate of $0.02-0.03$.
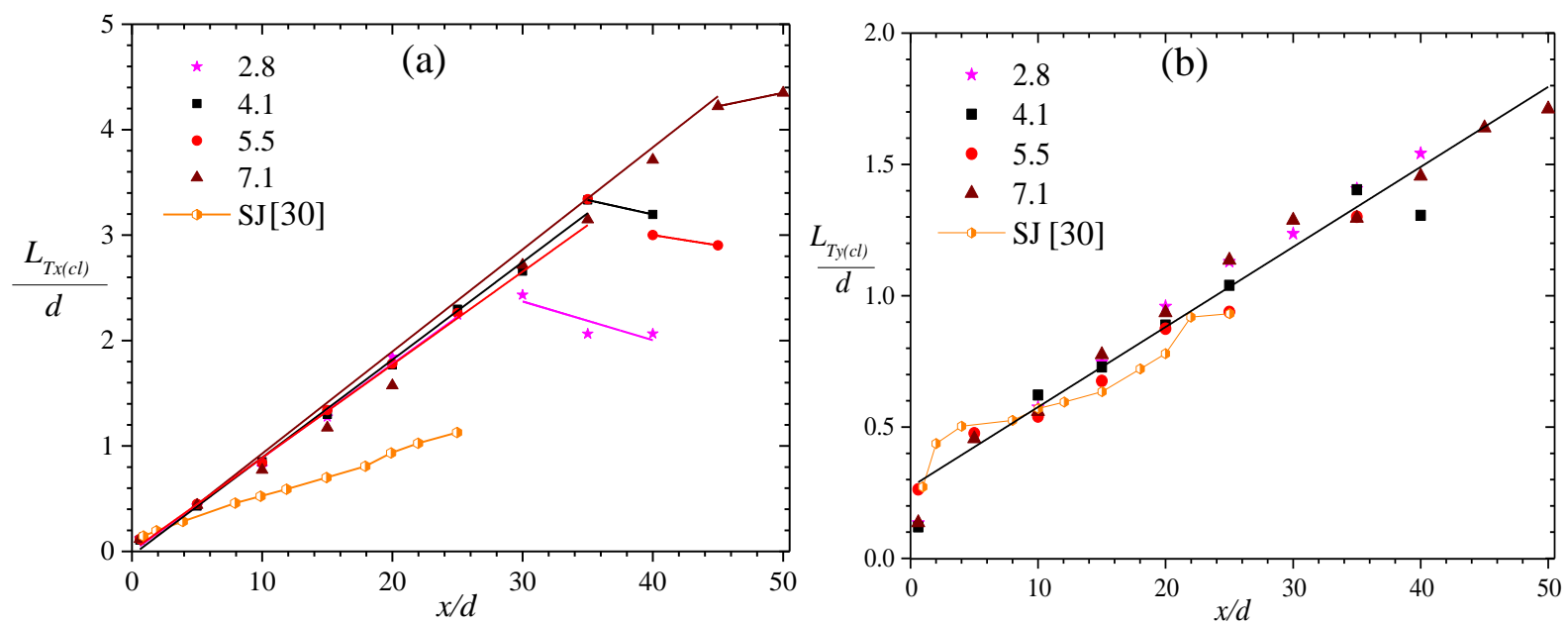

Figure 4.18: Variation of the (a) streamwise and (b) transverse integral length scales along the jet centerline

This behavior coincides with the centerline decay observed in Fig. 4.4, which shows the rate of jet centerline decay is significantly suppressed beyond the combined point. These results support the argument that highly coherent large-scale structures (indicated by large integral length scales) play a significant role in the engulfment of the surrounding ambient fluid, which in turn reduces the jet core velocity. The values of $L_{T y(c l)}$ also exhibit a linear growth, but with a $68 \%$ lower slope than that of $L_{T x(c l)}$. The growth rate of $L_{T y(c l)}$ coincides with the single jet growth rate, suggesting the large-scale structures grow at a similar rate in the transverse direction as that of the single jet.

The variation of streamwise and transverse integral length scales along the upper jet's outer (solid symbols) and inner (open symbols) half-velocity widths is presented in Fig. 4.19. It is shown that the values of $L_{T x(y 0.5)}$ and $L_{T y(y 0.5)}$ increase linearly with a slope of 0.157 and 0.040 , 
respectively, irrespective of shear layer and spacing ratio, noting that the differences in $L_{T}$ are within experimental uncertainty. These results suggest that the size of the large-scale structures along the half-velocity widths are independent of nozzle spacing. However, it is observed that the linear growth rate along the half-velocity widths is approximately $64 \%$ and $20 \%$ higher than its counterpart along the jet centerline for $L_{T x}$ and $L_{T y}$, respectively, indicating a stronger large-scale structure coherence along the shear layers than along the jet core.
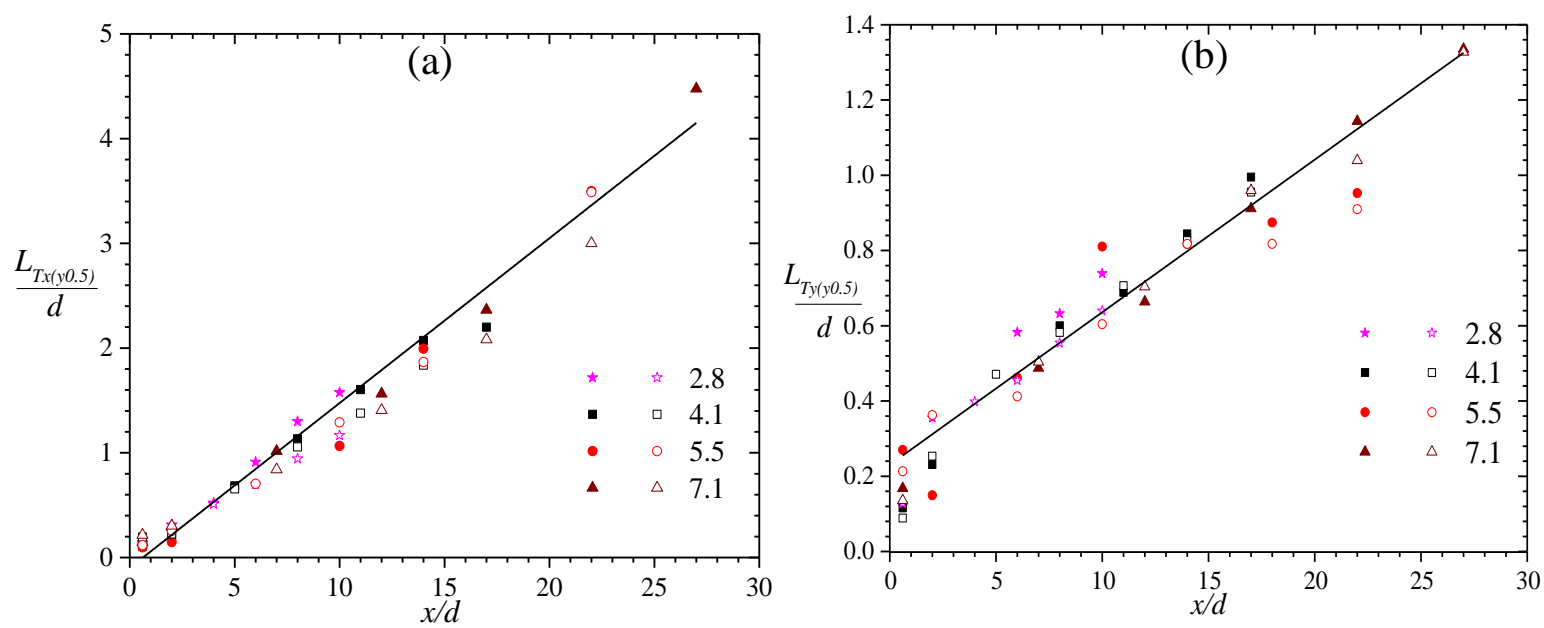

Figure 4.19: Variation of the (a) streamwise and (b) transverse integral length scales along the outer (solid symbols) and inner (open symbols) half-velocity widths 


\section{CHAPTER 5}

\section{CONCLUSIONS AND RECOMMENDATION FOR FUTURE WORK}

\subsection{Conclusion}

In summary, the effect of nozzle spacing on mean flow and turbulent characteristics of free round twin jets was investigated using a planar PIV. The experiments were performed at a fixed Reynolds number of 10,000 and for four different nozzle spacing ratios of $S / d=2.8,4.1,5.5$ and 7.1. The main conclusions of this study are as follows:

- Near the nozzle exit, the twin jets are isolated from each other and behave like a single jet, leading to a potential core length that is independent of nozzle spacing and similar to that of a free single round jet. Downstream of the potential core, a reduction in nozzle spacing leads to enhanced transverse mean velocity in the inner shear layers, and a significant rise of streamwise and transverse turbulent intensities and vorticity thickness along the symmetry plane. The development of streamwise mean velocity and turbulent intensities along the jet centerline is independent of nozzle spacing. However, the rise of transverse turbulent intensities along the centerline is enhanced for the smallest spacing ratio, $S / d=2.8$, relative to the larger spacing ratios.

- Both the merging and combined points' locations increase linearly with increasing nozzle spacing. Although this trend is similar to those found in previous round and plane twin jets, it was shown that the variation of $x_{m p}$ and $x_{c p}$ with streamwise distance is quite sensitive to initial conditions such as nozzle geometry and Reynolds number.

- In the converging region, the levels of the Reynolds normal stresses (particularly the transverse Reynolds normal stresses) in the inner shear layer are relatively higher than in the outer shear layer for the smallest spacing ratio $(S / d=2.8)$ that was not present in the higher spacing ratios. The enhancement of the Reynolds normal stresses was 
followed by their suppression in the inner shear layer, and the onset of suppression was delayed as the spacing ratio increased. The Reynolds shear stresses were also suppressed along the inner shear layer, particularly at lower spacing ratios. Even though the proximity of the two jets alters the turbulence characteristics in the inner shear layer, the levels of the Reynolds stresses in the outer shear layers are remarkably similar at all spacing ratios, which suggests that there exists no significant communication between the inner and outer layers, irrespective of nozzle spacing. Moreover, the interaction between the inner and outer shear layers did not displace the locations where the mean and Reynolds shear stress vanish relative to each other, and as a consequence, simple eddy viscosity models can be used to reliably predict twin round jets.

- In the immediate vicinity of the nozzle exit, the large-scale anisotropy along the jet centerline $\left(\overline{u_{c l}^{2}} / \overline{v_{c l}^{2}}\right)$ was at its highest level due to much lower $\overline{v_{c l}^{2}}$ levels relative to $\overline{u_{c l}^{2}}$. Subsequently, $\overline{v_{c l}^{2}}$ levels rise at a faster rate than $\overline{u_{c l}^{2}}$ leading to a reduction in $\overline{u_{c l}^{2}} / \overline{v_{c l}^{2}}$ to values close to unity at the end of the potential core length. Further downstream, $\overline{u_{c l}^{2}} / \overline{v_{c l}^{2}}$ stabilizes towards a value of $1.8 \pm 0.2$ for all spacing ratios.

- The skewness and flatness factor of the streamwise and transverse velocity fluctuations along the jet centerline show non-Gaussian distributions in the potential core region, but tend to Gaussian values beyond the potential core. While the skewness of the streamwise velocity fluctuations along the centerline is independent of spacing ratio, those of transverse velocity fluctuations are affected by nozzle spacing ratio. Along the symmetry plane, the streamwise and transverse skewness and flatness factors are sensitive to spacing ratio before the merging point, reflected by higher skewness and flatness at lower spacing ratios. Beyond the merging point, all profiles gradually approach Gaussian values. 
- Two-point correlation contours at the merging point showed that the spatial coherence of turbulence structures embodied in $R_{u u}$ increase with spacing ratio, serving as an indication that the vortical structures become more coherent with increasing spacing ratio at the onset of shear layer mixing. Along the jet centerline, the integral length in the streamwise direction increase linearly at two times the slope as the single jet linear growth rate, indicating that the vortical structures along the twin jet centerline are more coherent in the streamwise direction than that of the single jet. Beyond the combined point, the length scale growth rate drops and shrinks in a similar fashion to the centerline decay observed, supporting the argument that highly coherent large-scale structures play a significant role in the engulfment of the surrounding ambient fluid, which in turn reduces the jet core velocity. Along the half-velocity widths, the spatial coherence of the large-scale structures are independent of nozzle spacing. However, it is observed that the linear growth rate along the half-velocity widths is relatively higher than its counterpart along the jet centerline, indicating a stronger large-scale structure coherence along the shear layers than along the jet core.

\subsection{Recommendation for Future Work}

Some recommendations for future work on 3D free twin jets are summarized below:

- Due to limitations of the test facilities, the present study performed measurements in planes that are only parallel to the 3D flow, therefore, it was not possible to quantify the ambient fluid entrainment across the flow. Extracting data in planes that are also normal to the flow would allow an accurate quantification of the entrainment as it records the rate of change of the jet's mass flow rate.

- A proper orthogonal decomposition can be employed to extract the energetically dominant modes in the flow. The contribution of the extracted structures to the 
Reynolds stresses and their importance to turbulence production can then be examined.

- The planar PIV could not resolve the temporal evolution of the flow. A timeresolved PIV technique which is capable of providing the temporal evolution of the velocity field and the time-space correlations could be employed to study the time scales and other temporal characteristics of the coherent structures. 


\section{REFERENCES}

[1] Price Industries Ltd, 2011, Engineering Guide - Active and Passive Beams.

[2] Ball, C. G., Fellouah, H., and Pollard, A., 2012, “The Flow Field in Turbulent Round Free Jets,” Prog. Aerosp. Sci., 50, pp. 1-26 DOI: 10.1016/j.paerosci.2011.10.002.

[3] Pope, S. B., 2000, Turbulent Flows, Cambridge University Press, Cambridge.

[4] Deo, R. C., Mi, J., and Nathan, G. J., 2008, “The Influence of Reynolds Number on a Plane Jet,” Phys. Fluids, 20(7) DOI: 10.1063/1.2959171.

[5] Doglia, G., Romano, G. P., De Michele, G., and Tanzini, G., 1994, "LDA and PIV Velocity Measurements in Free Jets," Exp. Therm. Fluid Sci., 9(2), pp. 125-134 DOI: 10.1016/0894-1777(94)90105-8. [Accessed: 20-Jan-2017].

[6] Aleyasin, S. S., Fathi, N., Tachie, M. F., Koupriyanov, M., and Limited, P. I., 2017, "Experimental-Numerical Analysis of Turbulent Incompressible Isothermal Jets," ASME 2017 J. Fluids Eng. Division Summer Meeting, Waikoloa, Hawaii.

[7] Xu, M., Pollard, A., Mi, J., Secretain, F., and Sadeghi, H., 2013, "Effects of Reynolds Number on Some Properties of a Turbulent Jet from a Long Square Pipe,’ Phys. Fluids, 25(3) DOI: 10.1063/1.4797456.

[8] Fellouah, H., Ball, C. G., and Pollard, A., 2009, "Reynolds Number Effects within the Development Region of a Turbulent Round Free Jet,” Int. J. Heat Mass Transf., 52(1718), pp. 3943-3954.

[9] Aleyasin, S. S., Tachie, M. F., and Koupriyanov, M., 2017, "PIV Measurements in the near and Intermediate Field Regions of Jets Issuing from Eight Different Nozzle Geometries,” Flow, Turbul. Combust., 99(2), pp. 329-351 DOI: 10.1007/s10494-0179820-3.

[10] Quinn, W. R., 2006, "Upstream Nozzle Shaping Effects on near Field Flow in Round Turbulent Free Jets,” Eur. J. Mech. B/Fluids, 25(3), pp. 279-301 DOI: 
10.1016/j.euromechflu.2005.10.002.

[11] Mi, J., Kalt, P., Nathan, G. J., and Wong, C. Y., 2007, "PIV Measurements of a Turbulent Jet Issuing from Round Sharp-Edged Plate,” Exp. Fluids, 42(4), pp. 625637 DOI: 10.1007/s00348-007-0271-9.

[12] Cairns, C., Whitson, R. J., Strachan, P., and Wheel, M., 1994, "Prediction of Noise Generated by Orifice Plates in Liquid Systems Using a Modified Form of IEC,” Adv. Fluid Mech.

[13] Testud, P., Auré Gan, Y., Moussou, P., and Hirschberg, A., 2009, “The Whistling Potentiality of an Orifice in a Confined Flow Using an Energetic Criterion,” Jounral Sound Vib., 325, pp. 769-780 DOI: 10.1016/j.jsv.2009.03.046.

[14] Xu, G., and Antonia, R. A., 2002, "Effect of Different Initial Conditions on a Turbulent Round Free Jet,” Exp. Fluids, 33(5), pp. 677-683 DOI: 10.1007/s00348002-0523-7.

[15] Mi, J., and Nathan, G. J., 2009, "Statistical Properties of Turbulent Free Jets Issuing from Nine Differently - Shaped Nozzles," Flow Turbul. Combust, 84(4), pp. 583-606 DOI: 10.1007/s10494-009-9240-0.

[16] Aleyasin, S. S., Tachie, M. F., and Koupriyanov, M., 2016, “The Effects of Nozzle Geometry on the Behavior of Free Jets at Low Reynolds Number," ASME 2016 Fluids Engineering Division Summer Meeting, pp. 1-10.

[17] Miller, D. R., and Comings, E. W., 1959, "Force-Momentum Fields in a Dual-Jet Flow,” J. Fluid, 7(2), pp. 237-256 DOI: 10.1017/S0022112060001468.

[18] Lin, Y. E., and Sheu, M. J., 1990, “Investigation of Two Plane Parallel Unventilated Jets,” Exp. Fluids, 10, pp. 17-22.

[19] Nasr, A., and Lai, J. C. S., 1997, "Two Parallel Plane Jets: Mean Flow and Effects of Acoustic Excitation,” Exp. Fluids, 22(3), pp. 251-260 DOI: 10.1007/s003480050044. 
[20] Anderson, E. A., and Spall, R. E., 2001, "Experimental and Numerical Investigation of Two-Dimensional Parallel Jets,” ASME J. Fluids Eng., 123(June 2001), pp. 401-406 DOI: $10.1115 / 1.1363701$.

[21] Murai, K., Taga, M., and Akagawa, K., 1976, “An Experimental Study on Confluence of Two-Dimentional Jets,” Japanese Soc. Mech. Eng., 19(134), pp. 958-964.

[22] Okamoto, T., and Yagita, M., 1985, "Interaction of Twin Turbulent Ciruclar Jet," JSME, 28(238), pp. 617-622 DOI: 10.1248/cpb.37.3229.

[23] Meslem, A., Nastase, I., and Allard, F., 2010, "Passive Mixing Control for Innovative Air Diffusion Terminal Devices for Buildings,” Build. Environ., 45(12), pp. 26792688 DOI: 10.1016/j.buildenv.2010.05.028.

[24] Harima, T., Fujita, S., and Osaka, H., 2001, "Mixing and Diffusion Processes of Twin Circular Free Jets with Various Nozzle Spacing,” Experimental Heat Transfer, Fluid Mechanics, and Thermodynamics, pp. 1017-1022.

[25] Harima, T., Fujita, S., and Osaka, H., 2005, “Turbulent Properties of Twin Circular Free Jets with Various Nozzle Spacing,” ERCOFTAC International Symposium on Engineering Turbulence Modelling and Measurements, Italy, pp. 501-510 DOI: 10.1016/B978-008044544-1/50048-0.

[26] Gutmark, E. J., and Wygnanski, 1976, “The Planar Turbulent Jet,” J. Fluid Mech., 73(3), pp. 465-495.

[27] Nasr, A., and Lai, J. C. S., 1997, "Comparison of Flow Characteristics in the near Field of Two Parallel Plane Jets and an Offset Plane Jet,” Phys. Fluids, 9(10), pp. 29192931 DOI: $10.1063 / 1.869404$.

[28] Vouros, A., and Panidis, T., 2008, “Influence of a Secondary, Parallel, Low Reynolds Number, Round Jet on a Turbulent Axisymmetric Jet,” Exp. Therm. Fluid Sci., 32, pp. 1455-1467 DOI: 10.1016/j.expthermflusci.2008.03.007. 
[29] Aleyasin, S. S., Tachie, M. F., and Koupriyanov, M., 2017, "Statistical Properties of Round, Square and Elliptic Jets at Low and Moderate Reynolds Numbers," ASME J. Fluids Eng., 139(10), pp. 1-41 DOI: 10.1115/1.4036824.

[30] Aleyasin, S. S., Fathi, N., Tachie, M. F., Vorobieff, P., and Koupriyanov, M., 2018, “On the Development of Incompressible Round and Equilateral Triangular Jets Due to Reynolds Number Variation,” ASME J. Fluids Eng. DOI: 10.1115/1.4040031.

[31] Melling, A., 1997, "Tracer Particles and Seeding for Particle Image Velocimetry," Meas. Sci. Technol., 8(12), pp. 1406-1416 DOI: 10.1088/0957-0233/8/12/005.

[32] Prasad, A. K., 2011, Particle Image Velocimetry, Department of Mechanical Engineering, University of Delaware, Newark.

[33] Raffel, M., Willert, C. E., Wereley, S., and Kompenhans, J., 2012, "Particle Image Velocity A Practical Guide,” J. Vis. Exp., (70) DOI: 10.3791/4265.

[34] Adrian, R. J., 1986, “Image Shifting Technique to Resolve Directional Ambiguity in Double-Pulsed Velocimetry," Appl. Opt., 25(21), pp. 3855-3858.

[35] Johnson, R. W., and Smith, B. L., 2016, "Particle Image Velocimetry," Handbook of Fluid Dynamics, B.L. Smith, and D.R. Neal, eds., Taylor \& Francis Group, London, pp. 1-27.

[36] 2014, "DynamicStudio: User's Guide."

[37] Aleyasin, S. S., and Tachie, M. F., 2018, "Statistical Properties and Structural Analysis of Three-Dimensional Twin Round Jets Due to Variation in Reynolds Number,” Int. J. Heat Fluid Flow, (under review).

[38] Coleman, H. W., and Steelem, W. G., 1995, "Engineering Application of Experimental Uncertainty Analysis,” AIAA J., 33(10), pp. 1888-1896.

[39] Stern, F., Muste, M., Beninati, M. ., and Eichinger, W. E., 1999, Summary of Experimental Uncertainty Assessment Methodology with Example, Iowa City, IA. 
[40] Lan, K., and Jorgenson, J. W., 2001, “A Hybrid of Exponential and Gaussian Functions as a Simple Model of Asymmetric Chromatographic Peaks,” J. Chromatogr. A, 915(1-2), pp. 1-13 DOI: 10.1016/S0021-9673(01)00594-5.

[41] Ghahremanian, S., and Moshfegh, B., 2015, "Investigation in the Near-Field of a Row of Interacting Jets,” ASME J. Fluids Eng., 137(12), pp. 1-18 DOI: 10.1115/1.4031014.

[42] Brown, G. L., and Roshko, A., 1974, "On Density Effects and Large Structure in Turbulent Mixing Layers,” J. Fluid Mech., 64(4), pp. 775-816 DOI: 10.1017/S002211207400190X.

[43] Essel, E. E., and Tachie, M. F., 2015, "Roughness Effects on Turbulent Flow Downstream of a Backward Facing Step," Flow, Turbul. Combust., 94(1), pp. 125-153 DOI: $10.1007 / \mathrm{s} 10494-014-9549-1$.

[44] Essel, E. E., Nematollahi, A., Thatcher, E. W., and Tachie, M. F., 2015, "Effects of Upstream Roughness and Reynolds Number on Separated and Reattached Turbulent Flow,”J. Turbul., 16(9), pp. 872-899 DOI: 10.1080/14685248.2015.1033060.

[45] Abul Akon, 2017, "Effects of Turbulence on the Separating-Reattaching Flow above Surface-Mounted, Three-Dimensional Bluff Bodies," The University of Western Ontario.

[46] Hashiehbaf, A., and Romano, G. P., 2013, "Particle Image Velocimetry Investigation on Mixing Enhancement of Non-Circular Sharp Edge Nozzles,” Int. J. Heat Fluid Flow, 44, pp. 208-221 DOI: 10.1016/j.ijheatfluidflow.2013.05.017.

[47] Xu, M. Y., Tong, X. Q., Yue, D. T., Zhang, J. P., Mi, J. C., Nathan, G. J., and Kalt, P. A. M., 2014, "Effect of Noncircular Orifice Plates on the near Flow Field of Turbulent Free Jets,” Chinese Phys. B, 23(12) DOI: 10.1088/1674-1056/23/12/124703.

[48] Quinn, W. R., 2007, "Experimental Study of the near Field and Transition Region of a Free Jet Issuing from a Sharp-Edged Elliptic Orifice Plate,” Eur. J. Mech. B/Fluids, 
26(4), pp. 583-614 DOI: 10.1016/j.euromechflu.2006.10.005.

[49] Mi, J., Kalt, P., and Nathan, G. J., 2009, “On Turbulent Lets Issuing from NotchedRectangular and Circular Orifice Plates," Flow Turbul. Combust2, 84(4), pp. 565-582.

[50] Deo, R. C., Mi, J., and Nathan, G. J., 2005, "Dependence of a Plane Turbulent Jet on Its Nozzle Contraction Profile,” Proc. Int. Conf. Jets, Wakes Separated Flows, pp. 1-6.

[51] Mi, J., Nathan, G. J., and Nobes, D. S., 2001, "Mixing Characteristics of Axisymmetric Free Jets From a Contoured Nozzle, an Orifice Plate and a Pipe," ASME J. Fluids Eng., 123(4), pp. 878-883 DOI: 10.1115/1.1412460.

[52] Antonia, R., and Burattini, P., 2004, "Small-Scale Turbulence : How Universal Is It ?," 15th Australasian Fluid Mechanics Conference, Sydney.

[53] Deo, R. C., Mi, J., and Nathan, G. J., 2007, "The Influence of Nozzle Aspect Ratio on Plane Jets," Exp. Therm. Fluid Sci., 31(8), pp. 825-838 DOI:

10.1016/j.expthermflusci.2006.08.009.

[54] Banyassady, R., and Piomelli, U., 2015, "Interaction of Inner and Outer Layers in Plane and Radial Wall Jets,” J. Turbul., 16(5), pp. 460-483 DOI:

10.1080/14685248.2015.1008008.

[55] Abrahamsson, H., Johansson, B., and Lofdahl, L., 1997, The Turbulence Field of a Fully Developed Three-Dimensional Wall Jet, Sweden.

[56] Padmanabham, G., and Lakshmana Gowda, B. H., 1991, "Mean and Turbulence Characteristics of a Class of Three-Dimensional Wall Jets-Part 1: Mean Flow Characteristics,” ASME J. Fluids Eng., 113(4), pp. 620-628 DOI: 10.1115/1.2926525.

[57] Christensen, K., and Wu, Y., 2005, "Characteristics of Vortex Organization in the Outer Layer of Wall Turbulence," Fourth International Symposium on Turbulence and Shear Flow Phenomena, Vol. 3, Williamsburg, Virginia, pp. 1025-1030.

[58] Volino, R. J., Schultz, M. P., and Flack, K. a., 2009, “Turbulence Structure in a 
Boundary Layer with Two-Dimensional Roughness," J. Fluid Mech., 635, p. 75 DOI: $10.1017 / \mathrm{S} 0022112009007617$.

[59] Tay, G. F. K., Kuhn, D. C. S., and Tachie, M. F., 2015, "Effects of Sedimenting Particles on the Turbulence Structure in a Horizontal Channel Flow," Phys. Fluids, 27(2) DOI: 10.1063/1.4907669. 


\section{APPENDIX A}

\section{Convergence Test}

Before acquiring the data for each set of experiments, a convergence test was employed to determine the sample size required to accurately measure the mean velocity and higher order turbulent statistics reported in the study. In the general, higher order statistics require larger sample sizes. Also, the measurement uncertainty is thought to reduce with a larger sample size. A sample size of $N=1,000,2,000,3,000,4,000,5,000$ and 7,500 were used to calculate the mean velocity, Reynolds stresses (second order statistics), skewness (third order statistic) and flatness (fourth order statistic) in the converging region $(x / d=3)$ and the merging region $(x / d$ =7). Figure A.1 shows the results of the convergence test, including error bars on peak value data to objectively assess the data convergence. As can be observed, the differences among the mean velocity profiles obtained from the various sample sizes are within measurement uncertainty. This implies that $N \geq 1,000$ is sufficient to obtain statistical convergence of the mean velocity. For the Reynolds normal and shear stresses, on the other hand, the convergence of the peak values is reached at $N \geq 2,000$. In the case of the skewness factor, $N \geq 5,000$ provides a satisfactory statistical convergence, while, for the flatness factor, the statistical convergence shifts between $N=5,000$ and 7,500, depending on the location. As per these observations, 7,500 image pairs were acquired in each measuring plane in this study to ensure that higher-order statistics are resolved satisfactorily. 

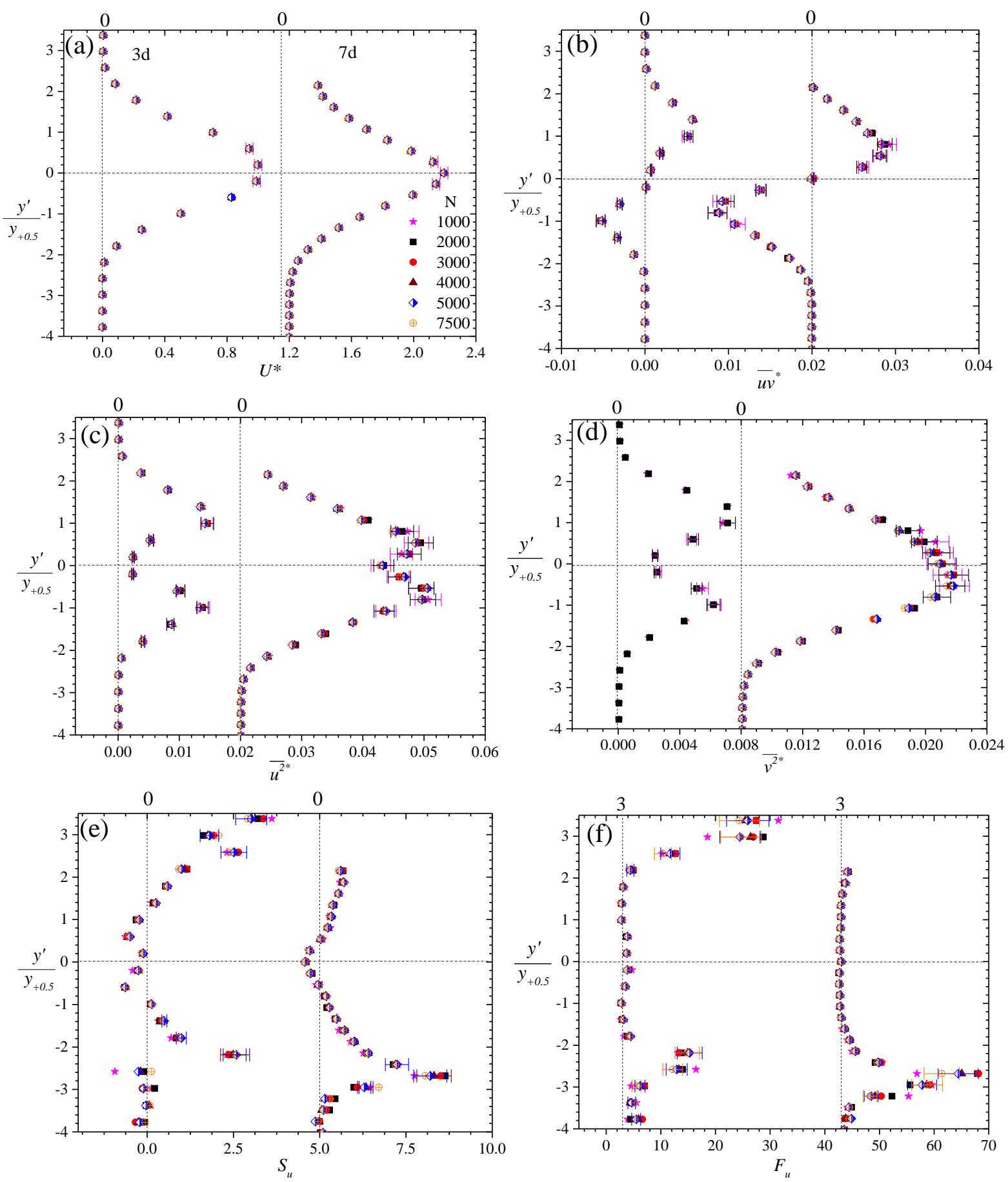

Figure A.1: Profiles of dimensionless (a) streamwise mean velocity, (b) Reynolds shear stress, (c,d) Reynolds normal stresses, (e)skewness, and (f) flatness factors for $N=1,000$, $2,000,3,000,4,000,5,000$ and 7,500 at $x / d=3$ and 7 


\section{APPENDIX B}

\section{Measurement Uncertainty}

\section{B.1 Systematic and Random Error Analysis Procedure}

A complete uncertainty analysis of the PIV measurement was performed following the procedure outlined by [38] and [39]. The procedure is outlined as follows: for a given measurement system, if a measured variable, $R$, can be expressed as a function of independent variables, $X_{1}, X_{2}, X_{3}, \ldots, X_{n}$, the total uncertainty $E_{R}$ in $R$ can be quantified as

$$
E_{R}^{2}=B_{R}^{2}+P_{R}^{2}
$$

where $B_{R}$ and $P_{R}$ are the total systematic and random errors in $R$ caused by uncertainties in the determination of the $X_{i}^{\prime}$ 's. The combined systematic errors in the independent variables are found by expanding $B_{R}$. Dropping higher order terms in the expansion would result in expressions of the form

$$
B_{R}^{2}=\sum_{i=1}^{n} \theta_{i}^{2} B_{i}^{2}+2 \sum_{i=1}^{n-1} \sum_{k=i+1}^{n} \theta_{i} \theta_{k} B_{i k}^{2}
$$

where $\theta_{x}=\partial R / \partial X_{i}$ are called sensitivity coefficients, $B_{i}$ is the systematic uncertainty limit in $X_{i}$, and $B_{i k}$ is the correlated systematic uncertainty limits in $X_{i}$ and $X_{k}$. In the present analysis, the systematic uncertainty limits were obtained from the manufacturer's specifications supplied with the PIV system.

The random error $P_{R}$ of the measured variables was calculated using the alternate approach suggested by [39]. In the alternate approach, assuming correlated random uncertainties, $P_{R}$ can be obtained from repeatability tests on $R$. If $S_{R}$ is the standard deviation of $K$ samples of $R$, then $P_{R}$ is given by 


$$
P_{R}=\frac{t S_{R}}{\sqrt{n}}
$$

where $t$ is derived from a Student $t$ distribution. For a large number of samples $(n \geq 10), t$ $=2$ at the $95 \%$ confidence level. The standard deviation is calculated from:

$$
S_{R}=\left[\sum_{k=1}^{M} \frac{\left(R_{k}-\bar{R}\right)^{2}}{n-1}\right]^{\frac{1}{2}}
$$

where $\bar{R}$ is the mean value of the $\mathrm{n}$ samples of $\mathrm{R}$ given by:

$$
\bar{R}=\frac{1}{M} \sum_{k=1}^{M} R_{i}
$$

\section{B.2 Uncertainty in the Mean Velocity}

In the present measurements, the systematic error in the mean velocity is considered to be of the same order as the error in the instantaneous velocities. In PIV, the instantaneous velocity at a point is the average fluid velocity over an interrogation area and is given by

$$
U_{i}=\frac{\Delta s L_{0}}{\mathrm{~L}_{\mathrm{i}} \Delta t}
$$

where $i=1$ and 2, $\Delta t$ is the time between laser pulses, $\Delta s$ is the particle displacement from the correlation algorithm, $L_{0}$ is the digital image width (in pixels) and $L_{i}$ is the field of view width of the camera (in meters). Using Eqn. (B.2) and assuming no correlated systematic uncertainty limits in the independent variables, the systematic uncertainty limit in $U_{i}$ is given by:

$$
B_{U i}^{2}=\theta_{\Delta s i}^{2} B_{\Delta S i}^{2}+\theta_{\mathrm{M}}^{2} B_{\mathrm{M}}^{2}+\theta_{\Delta t}^{2} B_{\Delta t}^{2}
$$

The sensitivity coefficients are given by:

$$
\theta_{\Delta S_{i}}=\frac{\partial U_{i}}{\partial \Delta S_{i}}=\frac{1}{M \Delta t}, \theta_{M}=-\frac{\Delta S}{M^{2} \Delta t} \text { and } \theta_{\Delta t}=-\frac{\Delta S}{M \Delta t^{2}}
$$


where $\mathrm{M}$ is the magnification factor of the camera, given by:

$$
M=\frac{L_{i}}{L_{0}}
$$

The uncertainty estimate of the mean velocity $U$ was calculated along the lower jet's outer shear layer $(y / d=-2.1)$, the inner shear layer $(y / d=-0.87)$, centerline $(y / d=-1.5)$ and the symmetry plane $(y / d=0)$ at $x / d=8$, and the most conservative value was selected to be the governing systematic uncertainty. The procedure is illustrated in Table B.1 for $S / d=2.8$ at the jets' symmetry plane as a representative case, along with the manufacturer's specifications for the systematic uncertainty limits in $\Delta s, \Delta t, L_{0}$ and $L_{i}[36]$

Table B.1 Systematic uncertainty limits of mean velocity at the jets' symmetry plane $(x / d=8$ $\& y / d=0)$

\begin{tabular}{|c|c|c|c|c|c|}
\hline Variable & Magnitude & $B_{x}$ & $\theta_{x}$ & $B_{x} \theta_{x}$ & $\left(B_{x} \theta_{x}\right)^{2}$ \\
\hline$L_{0}(m)$ & 0.1002 & $5.00 \mathrm{E}-04$ & $5.93 \mathrm{E}+01$ & $2.97 \mathrm{E}-02$ & 8.79E-04 \\
\hline$L_{i}($ pix $)$ & 2048 & $5.00 \mathrm{E}-01$ & $-4.34 \mathrm{E}-03$ & $-2.17 \mathrm{E}-03$ & $4.71 \mathrm{E}-06$ \\
\hline$M(p i x / m)$ & 20439.12 & & & & \\
\hline$\Delta t(s)$ & 2.33E-05 & $1.00 \mathrm{E}-07$ & $-2.18 \mathrm{E}+06$ & $-2.18 \mathrm{E}-01$ & 4.77E-02 \\
\hline$\Delta s(p i x)$ & $2.42 \mathrm{E}+01$ & $1.27 \mathrm{E}-02$ & 2.10 & 2.67E-02 & 7.11E-04 \\
\hline \multirow[t]{3}{*}{$U(m / s)$} & 11.54 & & & & \\
\hline & & & & $\sum\left(B_{x} \theta_{x}\right)^{2}$ & 4.93E-02 \\
\hline & & & & $\% B_{R}$ & $\pm 2.38 \%$ \\
\hline
\end{tabular}

To compute the random uncertainty limit, a total of 7500 instantaneous images were captured and divided into 10 sub-data sets of 750 images per set, in order to compute the standard deviation in Eqn. B.4. This means the number of readings in this case is $n=10$. The values of $U$ at the lower jet's outer shear layer $(y / d=-2.1)$, the inner shear layer $(y / d=-0.87)$, centerline $(y / d=-1.5)$ and the symmetry plane $(y / d=0)$ at $x / d=8$ were used to calculate the radon uncertainty at these locations, following Eqns. (B.3), (B.4) and (B.5), and the most conservative value was selected to be the governing random uncertainty. The procedure is illustrated in Table B.2 for $S / d=2.8$ at the jets' symmetry plane as a representative case. The 
systematic and random uncertainties were then combined using Eqn. (B.1) to calculate the total experimental uncertainty in the mean velocity.

Table B.2 Random uncertainty of mean velocity at the jets' symmetry plane $(x / d=8 \& y / d=$

0)

\begin{tabular}{ccccc}
\hline$n$ & $U$ & $U_{\text {mean }}$ & $S_{R}$ & $\% P_{R}$ \\
\hline 1 & 2.737 & 2.7833 & 0.91 & $\pm 0.58 \%$ \\
\hline 2 & 4.219 & & & \\
\hline 3 & 2.407 & & & \\
\hline 4 & 2.652 \\
\hline 5 & 3.768 & & & \\
\hline 6 & 2.905 & & & \\
\hline 7 & 12.10 \\
\hline 8 & 11.53 & & & \\
\hline 9 & 13.28 & & & \\
\hline 10 & 11.37 & & & \\
\hline
\end{tabular}

